\title{
The physical and chemical properties of heteronanotubes
}

\author{
Paola Ayala* \\ Faculty of Physics, University of Vienna, Strudlhofgasse 4, A-1090 Vienna, Austria \\ Raul Arenal and Annick Loiseau \\ Laboratoire d'Etude des Microstructures, ONERA-CNRS, 92322 Châtillon, France \\ Angel Rubio \\ Nano Bio Spectroscopy Group and ETSF Scientific Development Centre, Department of \\ Materials Science, University of the Basque Country UPV/EHU, Centro Mixto CSIC- \\ UPV/EHU and DIPC, Avd. Tolosa 72, 20018 San Sebastián, Spain \\ and Fritz-Haber-Institut der Max-Planck-Gesellschaft, Berlin, Germany
}

Thomas Pichler

Faculty of Physics, University of Vienna, Strudlhofgasse 4, A-1090 Vienna, Austria

(Published 9 June 2010)

\begin{abstract}
Carbon nanotubes undoubtedly take a leading position in nanotechnology research owing to their well-known outstanding structural and electronic properties. Inspired by this, hybrid and functionalized tubular structures have been constructed via several modification paths that involve the presence of molecules, generation of defects, and partial or full replacement of the carbon atoms, always maintaining a nanotube structure. The possibilities are countless. However, this review is mainly dedicated to giving a fundamental insight into the concepts behind wall modification, doping, and formation of a carbon nanotube structure. Theoretical concepts and experimental achievements ranging from carbon nanotubes with low $\mathrm{B}$ or $\mathrm{N}$ doping to the new physics behind boron nitride nanotubes are covered. Furthermore, special attention is devoted to the bulk and local characterization tools employed with these materials, their suitability and limitations. The theoretical approaches to describing the physical and chemical properties of heteronanotubes are objectively analyzed versus the materials available at this moment.
\end{abstract}

DOI: $10.1103 /$ RevModPhys.82.1843

PACS number(s): 73.22.-f, 73.63.Fg, 78.67.Ch, 61.46.Np

\section{CONTENTS}

I. Introduction

1844

II. Substitutionally Doped C-SWNTs and

Heteronanotubes of $\mathrm{C}, \mathrm{B}$, and $\mathrm{N}$

A. Substitutional doping: $\mathrm{CN}_{x}$ and $\mathrm{CB}_{y}$ CNTs

B. Novel heteronanotubes of $\mathrm{B}, \mathrm{C}$, and $\mathrm{N}$

1. $\mathrm{BC}_{2} \mathrm{~N}$

2. $\mathrm{BC}_{3}$

3. $\mathrm{C}_{3} \mathrm{~N}_{4}$

4. Boron nitride nanotubes

III. Synthesis of Heteronanotubes

A. Chemical vapor deposition

B. Arc discharge

C. Laser ablation and vaporization

D. Other methods

1. Ion implantation

2. Substitution reactions

3. Ball milling

IV. Contemporary Experimental and Theoretical Methods for Accessing the Physics and Chemistry of Heteronanotubes

*Corresponding author. paola.ayala@univie.ac.at
A. Spatially resolved techniques

1. TEM bright-field conventional and high-resolution imaging

1855

2. Analytic transmission electron microscopy

1855

3. Scanning probe microscopy

B. Momentum- and/or energy-resolved techniques

1. Bulk-sensitive EELS

2. X-ray absorption and photoemission

3. Optical techniques: Optical absorption, Raman, and luminescence spectroscopy

C. Structural analytic techniques

1856

1856

1856

1857

1858

1859

1. X-ray diffraction

2. Electron diffraction

1859

1859

D. Transport

E. Theoretical tools

1. Structural calculations for heteronanotubes

1860

1860

1860

2. Theoretical methods for spectroscopic tools

1861

V. Structural and Morphological Analysis of

Heteronanotubes of $\mathrm{B}, \mathrm{C}$, and $\mathrm{N}$

1861

A. Overall morphology

1861

B. Determination of doping amounts and dopant distribution

1861

C. Bonding environments

VI. Tentative Growth Mechanisms

VII. Electronic Structure and Transport

A. N and B substitutionally doped C-SWNTs
1863

1865

1866

1866 
B. Heteronanotubes

1. Electronic structure of $\mathrm{BC}_{2} \mathrm{~N}$ and $\mathrm{BC}_{3}$

2. Electronic structure of BN-NTs

VIII. Optical Properties

A. N-doped C-SWNTs and $\mathrm{C}_{3} \mathrm{~N}_{4}$-SWNTs

B. B-doped C-SWNTs

C. $\mathrm{BC}_{2} \mathrm{~N}$ and $\mathrm{BC}_{3}$

D. $\mathrm{BN}$ nanotubes

1. Influence of the correlation effects of the absorption spectrum

2. Excitonic effects

3. Luminescence

IX. Assessment of the Application Potential

X. Summary and Future Perspectives

Acknowledgments

References

\section{INTRODUCTION}

From both theoretical and experimental standpoints, carbon nanotubes (NTs) have been proved to exhibit promising behavior for different kinds of application. One of the most interesting facts is that their performance is completely dependent on the atomic geometrical organization. If unfolded, these tubular structures could be pictured as planar-hexagonal arrangements of carbon atoms distributed as in a honeycomb lattice (graphene). If we consider a one-layered tube, we are dealing with the structure known as a single-walled (SW) nanotube; multiwalled (MW) nanotubes are also under active investigation. These are a collection of concentric SW tubes with different diameters and their properties are different from those of their single-walled counterparts. Nevertheless, both kinds of structure represent a type of one-dimensional (1D) structure suitable for the investigation of mesoscopic phenomena. In this review, we focus strictly on single-walled carbon nanotubes and their possible modifications by the presence of nitrogen and boron as heteroatoms. As a special case, singlewalled boron nitride nanotubes (BN-SWNTs) are also analyzed as if a full substitution of the carbon atoms by $\mathrm{B}$ and $\mathrm{N}$ occurred. All these SWNTs that contain C, B, and $\mathrm{N}$ atoms exhibit different properties depending on the proportions of each heteroatom composing the tubular structure.

The properties of a physical system depend on its dimensionality, so in principle, at the nanoscale, the position of each atom influences the structural behavior as a whole. The electronic properties of C-SWNTs vary; depending upon the wrapping angle and diameter of the graphene sheet, a metallic or semiconducting 1D object can be formed (Hamada et al., 1992; Mintmire et al., 1992; Dresselhaus et al., 1995; Saito, 1996). However, this does not necessarily apply to all kinds of heteronanotube with a hexagonal wall structure containing $\mathrm{C}, \mathrm{B}$, and $\mathrm{N}$. The electronic structure of $\mathrm{C}$ and $\mathrm{BN}$ nanotubes can be constructed starting from the electronic structure of the graphene or $h$-BN single sheet, respectively, via the well-known zone-folding procedure. This has been explained in the literature [related information can be

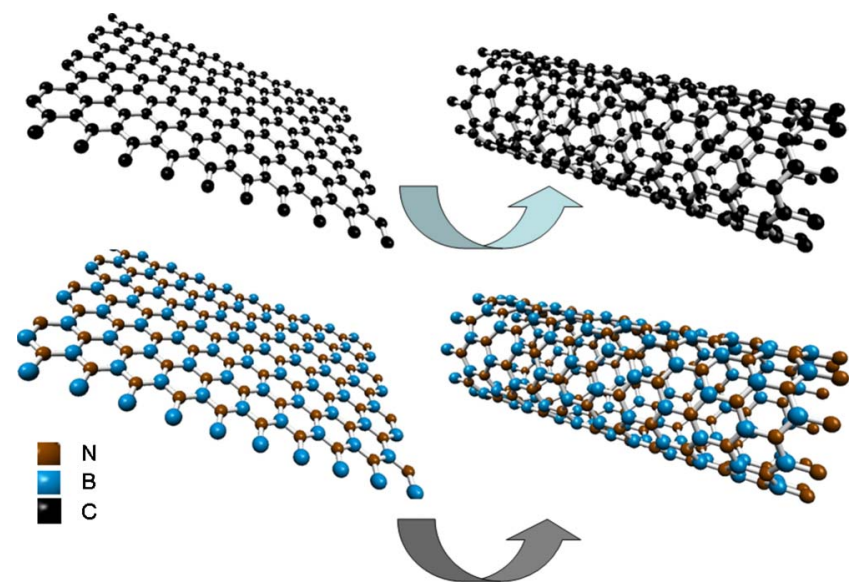

FIG. 1. (Color online) Structure of a single-walled carbon nanotube pictured as a rolled-up graphene sheet (top). In the same manner, a BN nanotube would be the corresponding tubular structure related to a stable planar $h$-BN sheet (bottom).

found, for instance, in Saito et al. (1998), Dresselhaus et al. (2001), Jorio et al. (2008), and Yap (2008)]. The band structure of a C-SWNT can be approximated by imposing periodic boundary conditions on the band structure of planar graphene. The same principle can be applied to a BN-SWNT, which is also a tubular structure, but based on a hexagonal graphitelike $\left(s p^{2}\right) \mathrm{BN}$ phase $(h$-BN) as shown in Fig. 1. Rubio et al. (1994) initially proposed this tubular structure based on the similarities between graphene and $h$-BN; and Blase et al. (1994) performed calculations on the electronic properties and the dependence on the tube diameter.

According to the zone-folding procedure, the band structure of the tube can be obtained by cutting the band dispersion of the sheet along certain parallel lines in the reciprocal space [vertical black lines in the grayshaded area in Fig. 2(b)]. The band structure of the sheet is characterized by a large direct gap at the $K$ point, whereas the band structure of a carbon sheet (graphene) has no gap at the $K$ point and a linear band dispersion (a zero-gap semiconductor). The spacing of the lines is determined by the quantization of the wave-vector component $k_{\perp}$ along the tube circumference. The different lines correspond to different angular momenta (quantum number $m$ ) along the axis of the tube. Comparing the band structure of the sheet to that of the tube, one sees that the highest valence band and the lowest conduction band of the tube can be directly obtained from the $\pi$ and $\pi^{*}$ bands of the sheet along the line $M \rightarrow K$ and beyond. A $h$-BN sheet and a $(3,3)$ armchair $\mathrm{BN}$ SWNT, with the corresponding band structures, are shown in Fig. 3. The wave functions of the $\pi$ and $\pi^{*}$ bands are predominantly composed of atomic $p_{z}$ orbitals (i.e., $p$ orbitals with an orientation perpendicular to the plane). The $\pi$-band wave function is predominantly located at the nitrogen atoms and the $\pi^{*}$-band wave function at the boron atoms; this is because of the higher electronegativity of nitrogen. It is the strong difference in electronegativity between $\mathrm{B}$ and $\mathrm{N}$ that leads to the 


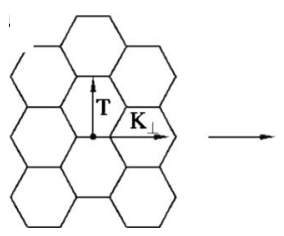

(a)

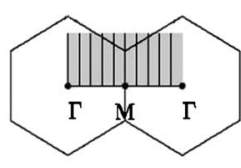

(b)
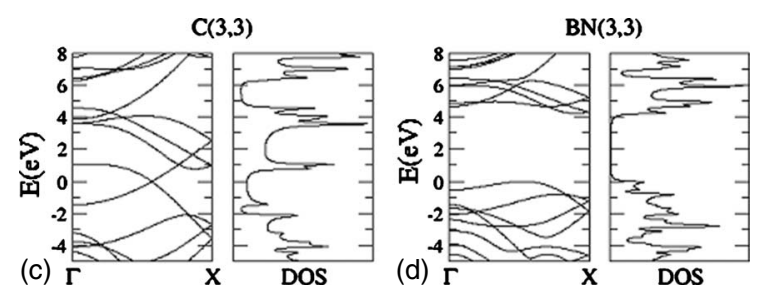

FIG. 2. The figures on the left show a simplified scheme of the hexagonal network in (a) real and (b) reciprocal spaces. This applies for the hexagonal lattices described in this review. The pictures on the right correspond to energy dispersion schemes and LDOS showing the van Hove singularities of a $(3,3)$ C-SWNT and a $(3,3)$ BN-SWNT (two different heteronanotubes with equivalent chirality).

large band gap. In a graphene sheet, where both atoms in the unit cell are equivalent, the $\pi$ and $\pi^{*}$ bands are degenerate at $K$, leading to the linear crossing of the two bands.

In contrast to C-SWNTs, BN-NTs are always semiconducting with a large band gap that is nearly independent of the tube diameter, chirality, and whether the NT is $\mathrm{SW}, \mathrm{MW}$, or packed in bundles. Another remarkable feature appears in small-diameter BN-SWNTs, where the $\mathrm{BN}$ wall system buckles, placing the $\mathrm{B}$ atoms inward and the $\mathrm{N}$ atoms outward, resulting in a dipolar doublecylinder shell structure.

Until now we have dealt with C- and BN-SWNTs, but the scope of this review envisages NTs with different stoichiometries composed of $\mathrm{B}, \mathrm{C}$, and $\mathrm{N}$ atoms. It is worth recalling that one route for modifying the solid state properties of materials is the addition of electron acceptors or donors. This is a well-known procedure in semiconductor technology for many other crystalline solid structures (Ashcroft and Mermin, 1976; Yu and Cardona, 2001). The electronic properties of NTs are different from those of bulk doped carbons, and even other
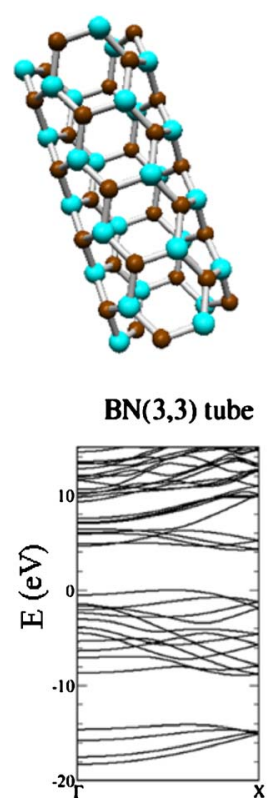
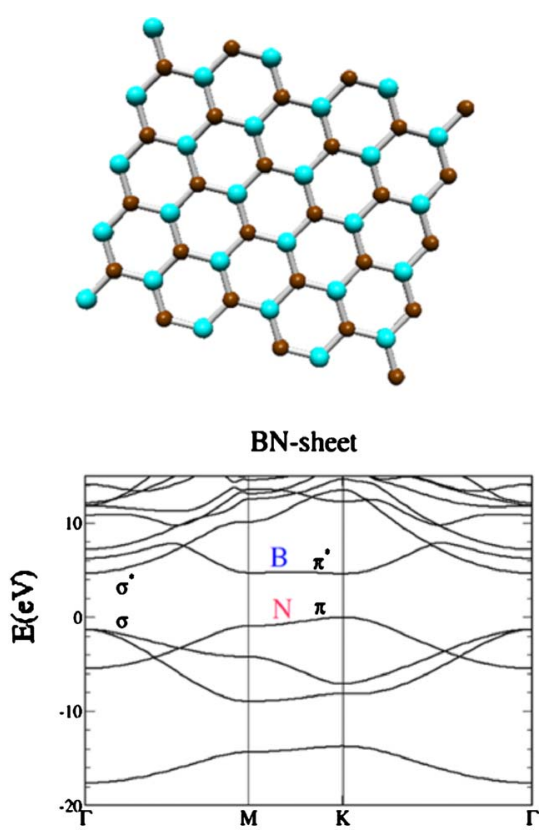

FIG. 3. (Color online) Comparative scheme of the electronic structure of a $\mathrm{BN}(3,3)$ nanotube and a $h$-BN sheet. The band structure of the nanotube. layered carbon structures (i.e., bulk doped graphite), because of the quantum confinement and curvature of the cylinders. The NT semiconducting properties can be affected by the position of the heteroatoms: Some atoms or molecules can also be encapsulated in the hollow core of the tubes (endohedral doping) or trapped within bundles intercalated between the outer shells of the tubes (exohedral doping). A simplified scheme of the types of functionalization is shown in Fig. 4. In this article we focus strictly on wall functionalization that involves substitutional doping with $\mathrm{B}, \mathrm{C}$, or $\mathrm{N}$ atoms, which implies direct substitution of carbon atoms in C-SWNTs, partial substitution of BN dipoles, or reorganization of the hexagonal lattice to incorporate heteroatoms in the wall structure.

Nitrogen contains one additional electron compared to carbon, and boron lacks one; thus novel electronic properties can be expected if $\mathrm{N}$ and $\mathrm{B}$ atoms directly substitute $\mathrm{C}$ atoms in the graphitic lattice of C-SWNTs. Both low- and high-doping regimes can be envisaged. However, if the heteroatoms that act as dopants exceed a certain amount, stable planar structures analogous to graphene but with defined stoichiometries containing B, $\mathrm{C}$, and/or $\mathrm{N}$ can be generated. Nanotubes formed by the rolling up of such structures can also be considered. The most studied such heteronanotubes are the $\mathrm{BC}_{3}$-SWNTs, but there are other stable planar stoichiometries that also exhibit the possibility to form NTs; they will be ex-
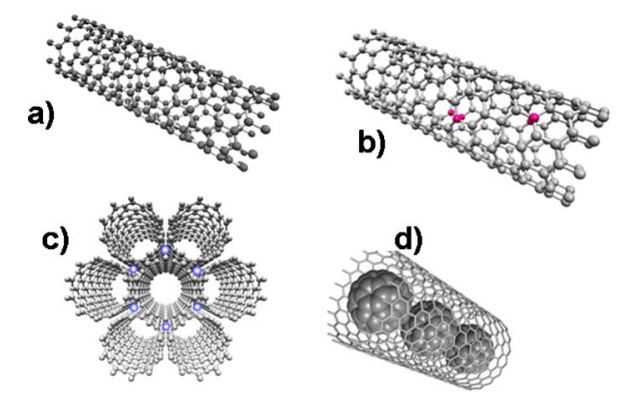

FIG. 4. (Color online) The molecular models show the different ways to modify the electronic properties of a pristine single-walled carbon nanotube. The diagrams represent (a) a pristine C-SWNT, (b) heteronanotubes with substitutional atoms, (c) intercalation within C-SWNTs in a nanotube bundle, and (d) endohedral doping by filling with different molecular structures such as the example depicted with encaged fullerenes in so-called peapods. 
plained later with emphasis on the correlation between theory and experiment.

We give special attention to the suitability of the techniques used for the characterization of these doped NTs and heteronanotubes. Transmission electron microscopy (TEM) in high-resolution mode as well as scanning tunneling microscopy (STM) and scanning tunneling spectroscopy (STS) make it possible to image the NTs with atomic resolution and to identify the nanotube geometry at the local scale. Near-field Raman spectroscopy also allows one to probe these structures with spatial resolution, which is lost in other spectroscopic methods. Additional information can be obtained with bulk spectroscopic methods regarding the vibronic and electronic structure and optical properties of the different samples. The list of characterization tools is extensive, and therefore an entire section of this review is devoted to characterization techniques and their appropriateness for studying these different materials.

We intend this contribution to provide an extensive theoretical and experimental review of the physical and chemical properties of heteronanotubes of $\mathrm{C}, \mathrm{B}$, and $\mathrm{N}$, constrained to the single-walled NT case. Carbon, boron nitride, and doped MWNTs are not dealt with in this review, since others have covered in detail in review articles the experimental and theoretical advances related to these structures, as well as chemical functionalization topics (Tenne and Zettl, 2001; Terrones et al., 2004; Golberg et al., 2007; Arenal et al., 2010). In the following sections we describe the basic concept of each type of heteronanotube of $\mathrm{C}, \mathrm{B}$, and $\mathrm{N}$, with an insight into the theoretical concepts in each case. The state-of-the-art synthesis methods are described in Sec. II, followed by a discussion of the methods utilized to access specific physical and chemical properties of these materials. Sections V-VIII are dedicated to the inherent characteristics predicted versus the experimentally observed phenomena. An assessment of the potential applications for these structures is given at the end as we expect to provide a strong fundamental basis, which makes the last section practically self-explanatory.

Before moving into the next sections it is useful to remark that the "SWCNT" and "MWCNT" abbreviations that stand for carbon single-walled and multiwalled carbon nanotubes are not used here as in most of the related literature. Throughout this review, "SWNT" will be used regardless of the atomic structure of the singlewalled nanotube. The type of heteronanotube will be identified with atomic symbols as prefixes. Note that a single-walled carbon nanotube has been abbreviated C-SWNT.

\section{SUBSTITUTIONALLY DOPED C-SWNTS AND HETERONANOTUBES OF C, B, AND N}

This section discusses the structural characteristics of heteronanotubes formed with $\mathrm{C}, \mathrm{B}$, and $\mathrm{N}$. The properties of carbon-based structures are intrinsically linked to the type of hybridization (i.e., $s p^{1}, s p^{2}$, and $s p^{3}$ ) in which the atoms bond with neighboring atoms (Saito et al.,
1998; Robertson, 2002). From this starting point it is easily inferable that, since carbon systems have been studied, nitrogen and boron have always been considered as natural dopants given their size proximity to $\mathrm{C}$ (Zanchetta and Marchand, 1965; Cermignani et al., 1995). The properties of nitrogen- and boron-containing $\left(\mathrm{CN}_{x}\right.$ and $\left.\mathrm{CB}_{y}\right)$ SWNTs change depending on the amount of foreign atoms in the structure. Keeping this in mind, it is important to start from a conceptual viewpoint: one may not always use the words "doped nanotube" for any carbon tubular structure that contains foreign ( $\mathrm{N}$ or $\mathrm{B})$ atoms. Picture the typical honeycomb model as a set of hexagons perfectly interconected. We are dealing here with graphitelike planar structures whose geometry resembles that of this honeycomb. Doped graphene can be considered as a structure in which every $\mathrm{C}$ atom in a corner of the hexagons is replaced by a dopant. However, if these dopant agents indeed enter, they can break the stability of the planar structure depending on where and how much is incorporated. In the same way that graphene can be thought of as the parent material of a C-SWNT, hexagonal graphitelike planar structures should be able to generate nanotubules with different stoichiometries based on B, C, and N. Doping considerations should be analyzed with the proper quantum confinement effects taken into account.

It would be ideal to place the doping agents directly in the corners of the hexagons, where the $\mathrm{C}$ atoms were originally. However, this does not happen straightforwardly. As mentioned, the stability of the structure can be compromised, depending on the number of atoms replaced. There will also be some favorable surrounding elemental configuration when a guest atom enters the structure. This is undoubtedly far from being a triviality, and this section is devoted to clarifying it.

\section{A. Substitutional doping: $\mathrm{CN}_{x}$ and $\mathrm{CB}_{y}$ CNTs}

Substitutional doping by heteroatoms is a wellestablished key technique in Si technology, since doping with electron donors or acceptors leads to a sensitive shift of the Fermi level. In all semiconductor applications, the properties of a device depend on control of the electronic states in the valence and conduction bands for design and optimization purposes. However, the doping levels for such applications are on the order of parts per million (Yu and Cardona, 2001).

In this context, C-NTs, which have been well studied in recent years, are strong candidates for applications in nanobased semiconductor technology. However, optimized control of the electronic properties is still far from attained. One of the proposed solutions to control the electronic behavior of the nanotubes is to dope them with donors or acceptors. The first studies analyzing doping effects on nanotubes appeared in 1993 (Yi and Bernholc, 1993), not much later than Iijima's first reports on TEM observations (Iijima, 1991; Iijima and Ichihashi, 1993). Doping of C-NTs with boron and nitrogen may lead to electronic properties that are more controlled by 


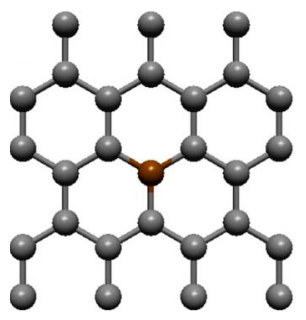

(a)

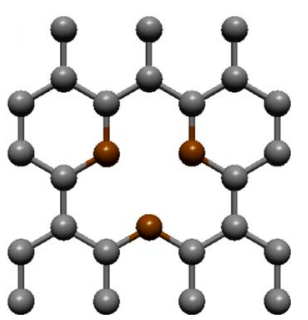

(b)

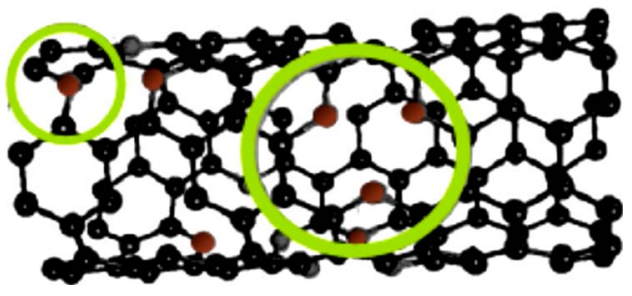

(c)

FIG. 5. (Color online) N (represented by circles) can be incorporated in the walls of the nanotube in different ways. The most probable configurations for $\mathrm{N}$ in the graphitic wall of a C-SWNT are (a) direct substitution or (b) pyridinelike bond. (c) The stability of a nanotube with both type of incorporation is still a matter of discussion.

the chemistry than by the specific geometry of the tubes. Thus the heteroatoms inserted in the C-SWNT structure must be present up to certain doping levels and their bonding environments must be controlled.

We first analyze the case of nitrogen as a dopant. It has one electron extra compared to $\mathrm{C}$ and, from an electronic point of view, it is natural to expect an excess of donors in the N-rich areas of CNTs [see Fig. 5(a)]. If N substitutes for $\mathrm{C}$ directly, an $n$-type semiconductor with sharp localized states above the Fermi level is generated. Because of its size, $\mathrm{N}$ can also generate a defect in the tube structure, keeping the heteroatoms on the walls but requiring a rearrangement of the neighboring $\mathrm{C}$ atoms. In this case, it is not immediately obvious that $n$-type behavior will result, as in the case of $\mathrm{N}$ direct substitution. A second configuration involving different wall rearrangements might occur. The electronic behavior then depends on the new geometry generated, and this new wall structure could be such that $p$-type doping is also feasible. The most probable configurations for $\mathrm{N}$ substituted in the wall of a C-SWNT are the direct replacement of a carbon atom [see Fig. 5(a)] and a pyridinic type [see Fig. 5(b)]. This will be discussed in more detail in Sec. VII, devoted to electronic structure and transport.

Unlike N, B is expected primarily to substitute directly for $\mathrm{C}$ atoms with a triple-coordinated bond. Nevertheless, the formation of nanodomains of $\mathrm{B}$ along the tubular structure cannot be discounted, again inducing changes in the electronic structure of the nanotube (Carroll et al., 1998; Quandt et al., 2008). Low concentrations of B atoms should lead to the formation of an acceptor state in the band gap at low energy above the valence edge. Calculations from Yi and Bernholc (1993) found an acceptor state located at $0.16 \mathrm{eV}$ above the Fermi energy for an $(8,0)$ nanotube, where the $\mathrm{B}: \mathrm{C}$ ratio employed was $1: 80$. Further studies by Wirtz and Rubio (2003) discussed the evolution of this acceptorlike level with the degree of $\mathrm{B}$ doping, pointing out that low and high doping entail critical differences in the physical properties. In the case of undoped C-SWNTs, the density of states (DOS) is symmetric around the band gap for the first and second van Hove singularities (vHss). However, with the introduction of $\mathrm{B}$, asymmetries arise around the band gap owing to the mixing of the $p$ and $s$ orbitals. Thus this doping induces a lowering of the
Fermi level into the valence band of the undoped tube.

Now it is worth considering the concept of the rigid band model and its validity in doped C-SWNTs. This is particularly important when analyzing the properties of doped nanotubes, since doping amounts on the order of parts per million allow consideration of a rigid band model, which assumes that the electronic DOS of a structure can be inferred from that of the host. In other words, for nanotube systems with low doping, any band structure modification can be considered negligible and the band structure of the doped system can be obtained by shifting the Fermi level in the band structure of the pristine system toward the valence or conduction band. The applicability of a rigid band model has been proven for nanotubes by field-effect doping ( $p$ - $n$ type) and intercalation with alkali metals even to very high doping levels $(10 \%) n$ type, and for $p$-type doing for $\mathrm{Br}_{2}, \mathrm{I}_{2}$, and $\mathrm{FeCl}_{3}$ doping (Lee et al., 1997; Kataura et al., 2000; Suzuki et al., 2003; Liu et al., 2004). For low doping levels of substitutional heteroatoms in CNTs, the rigid band model is most likely to be valid since these structures are similar to doped Si with only a small number of dopantrelated defect centers. However, at higher doping, when heteronanotubes are formed, the story gets more complicated, and modifications in the DOSs of these substitutional dopants have to be taken into account. This means that a simple rigid band shift model is no longer applicable because novel heteronoanotubes are created.

\section{B. Novel heteronanotubes of $\mathbf{B}, \mathbf{C}$, and $\mathbf{N}$}

In semiconductor physics, doping levels are on the order of parts per million. However, when greater heteroatom incorporation occurs, the concept of a $\mathrm{CN}_{x}$ or a $\mathrm{CB}_{y}$ heteronanotube must be taken into account carefully as two possible situations must be considered: high doping and formation of novel heteronanotubes.

As starting point we defined a heteronanotube of $\mathrm{B}$, $\mathrm{C}$, and $\mathrm{N}$ as a one-dimensional tubular structure with essentially similar overall morphology to that of a C-SWNT: a tubule with a honeycomblike wall structure. This wall structure, namely, a rolled graphene layer in the case of a C-SWNT, is in principle a stable planar layer of carbon atoms arranged in a hexagonal distribution. Just as a carbon nanotube can be pictured as a 


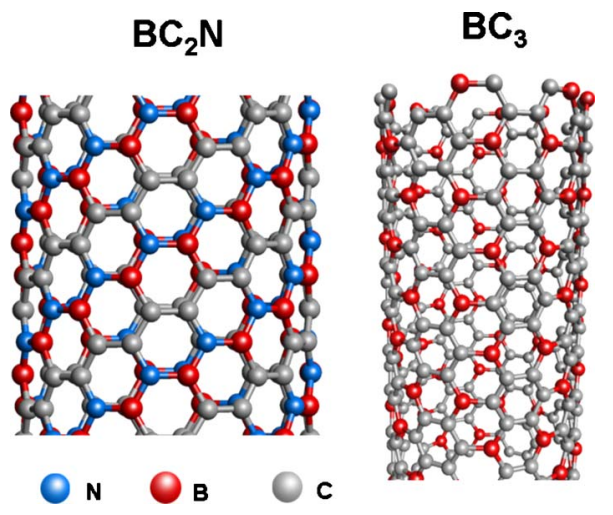

FIG. 6. (Color online) Molecuar models of a $\mathrm{BC}_{2} \mathrm{~N}$ nanotube (left) and a $\mathrm{BC}_{3}$ nanotube (right).

rolled-up graphene sheet, in the same manner it should be possible to form nanotubes from other planar structures containing different atoms; of course, taking into account energetic considerations for the stability limits of tubular structures upon curvature of the walls. Planar heterostructures with a similar geometry to graphene, and which contain heteroatoms such as $\mathrm{N}$ or $\mathrm{B}$, have been predicted theoretically. Some introduction to this topic can be found in Tenne and Zettl (2001) and Terrones et al. (2002). The stability of the planar arrangement of such structures could make it possible for a nanotube with the same stoichiometry to exist. Here we describe the most commonly studied examples, but emphasize that other nanotube structures containing B, C, and $\mathrm{N}$ might be possible.

\section{1. $\mathrm{BC}_{2} \mathrm{~N}$}

One $\mathrm{B}, \mathrm{C}$, and $\mathrm{N}$ combination that crystallizes in a graphitelike planar structure is $\mathrm{BC}_{2} \mathrm{~N}$. Miyamoto, $\mathrm{Ru}-$ bio, Cohen, and Louie (1994) performed first-principles and tight-binding band structure calculations to analyze the stability of families of nanotubes originating from hexagonal stable $\mathrm{BC}_{2} \mathrm{~N}$ sheets (Liu et al., 1989) and suggested that chiral nanotube formation is preferential to that of graphite and BN. Nanotubes originating from these hexagonal sheets are expected to be systems with intermediate stabilities between those of graphite and $h$-BN. A molecular model of one possible $\mathrm{BC}_{2} \mathrm{~N}$ nanotube is shown in Fig. 6. This is the most stable geometry and it has been calculated as semiconducting, as explained by Miyamoto and co-workers. They also studied the effects of a $\mathrm{C}$ impurity located on either the $\mathrm{N}$ or the $\mathrm{B}$ site. The energy band structures corresponding to these situations are discussed later and shown in greater detail in Fig. 28.

We emphasize that the $\mathrm{BC}_{2} \mathrm{~N}$ arrangement is one possibility, but others, such as Mazzoni et al. (2006), have also investigated the relative stability of several layered structures of $\mathrm{B}, \mathrm{N}$, and $\mathrm{C}$. In this work, they used firstprinciples calculations and suggested that a $\mathrm{B}_{3} \mathrm{C}_{2} \mathrm{~N}_{3}$ structure could be more stable than $\mathrm{BC}_{2} \mathrm{~N}$.
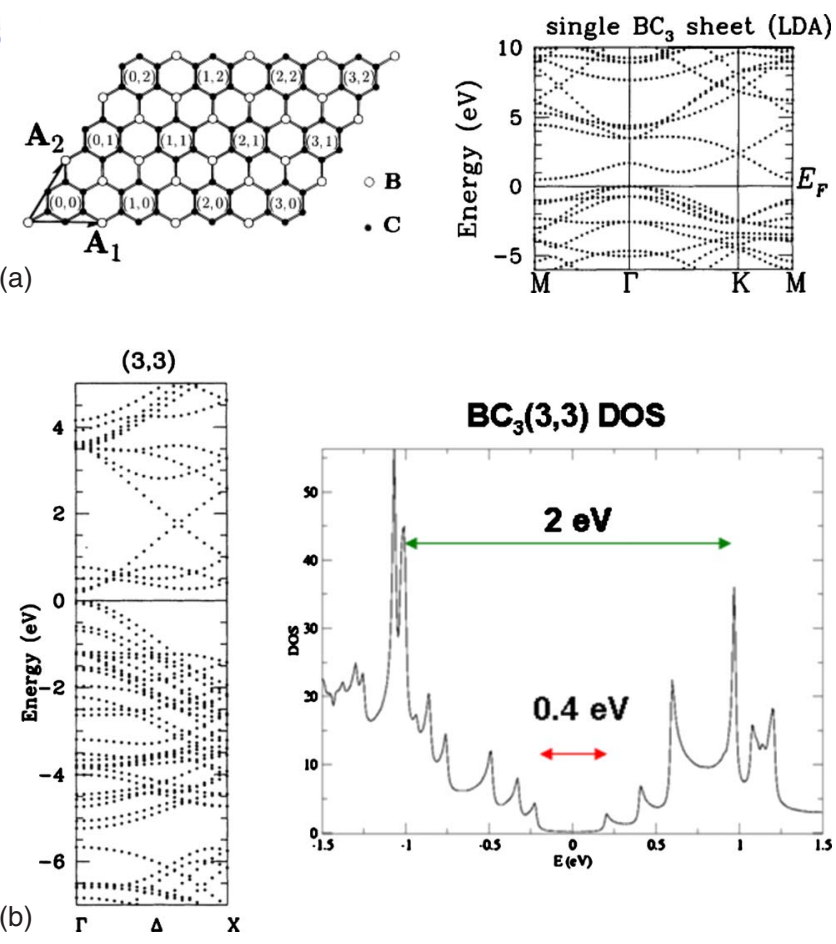

FIG. 7. (Color online) The $\mathrm{BC}_{3}-\mathrm{SWNT}$ can be thought as a planar rolled-up sheet of the same stoichiometry. The upper panel shows a single $\mathrm{BC}_{3}$ planar graphitelike sheet with the corresponding band structure proposed by Miyamoto, Rubio, Louie, and Cohen, 1994. The lower panels represent the band structure and density of states of a $(3,3) \mathrm{BC}_{3}$-SWNT. This has been adapted from Miyamoto, Rubio, Louie, and Cohen, 1994.

\section{2. $\mathrm{BC}_{3}$}

Here we are dealing with the case of $\mathrm{BC}_{3}$-SWNTs, which are not highly B-doped SWNTs. These structures can be regarded within the framework of heteronanotubes rather than doped nanotubes, as rolled-up $\mathrm{BC}_{3}$ planar graphitelike stable sheets. On these grounds, it is reasonable to envisage a $\mathrm{B}$ content as high as $15 \%$, disregarding the solubility limits for boron in carbons as some suggest (Serin et al., 2000; Terrones et al., 2004).

This is not the $\mathrm{B}$ percentage required to form a nanotube with the $\mathrm{BC}_{3}$ stoichiometry; this will be discussed further in the section of electronic properties of $\mathrm{BC}_{3}$ nanotubes. Experimental results on $\mathrm{BC}_{3}$ nanotubes reported by Fuentes et al. (2004) showed the formation of a uniform energy gap of $0.4 \mathrm{eV}$ for B-doped nanotubes at very high doping. They confirmed the existence of $\mathrm{BC}_{3}$ nanotubes with an acceptorlike band of $\sim 0.1 \mathrm{eV}$ above the Fermi level (see Fig. 7). This confirms that for B-doped nanotubes at low doping a rigid band model can be applied, but at higher boron incorporation new stable structures are observed. The exact doping level up to which a rigid band model is still applicable remains an open question. Carroll et al. (1998) proposed that B substitution in C-NTs leads to the formation of $\mathrm{BC}_{3}$ nanodomains, which give rise to an acceptor state near the Fermi level. 


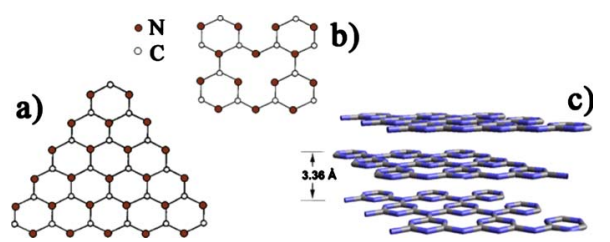

FIG. 8. (Color online) $\mathrm{C}_{3} \mathrm{~N}_{4}$ is one of the promising stable graphitelike structures that contain N. It is expected that these structures form nanotubes of the same stoichiometry. Depicted are the (a) Redemanns model and (b) Alves model for graphitelike $\mathrm{C}_{3} \mathrm{~N}_{4}$ as described in Komatsu (2001). (c) Multiplane stacking of $\mathrm{C}_{3} \mathrm{~N}_{4}$ layers exhibiting an interplanar distance close to graphite.

\section{3. $\mathbf{C}_{3} \mathbf{N}_{4}$}

Following the same considerations as in the previous two cases, we expect graphitelike $\mathrm{C}_{3} \mathrm{~N}_{4}$ arrangements to generate tubular structures, as this stoichiometry gives rise to several configurations that exhibit planar stable forms (see Fig. 8) (Teter and Hemley, 1996).

For $\mathrm{C}_{3} \mathrm{~N}_{4}$ the implications and limits of the rigid band model have still not been analyzed. Nevertheless, in this case it is anticipated that the band structure will exhibit completely different features (Gracia and Kroll, 2009). Whether it is possible to synthesize low-doped SWNTs or go to higher doping levels that allow the formation of $\mathrm{C}_{x} \mathrm{~N}_{y}$ nanotubes (or any other stable $\mathrm{N}-\mathrm{C}$ planar structure) is one of the crucial concerns in this research area (Kroke and Schwarz, 2004). The question involves a number of factors that embrace theoretical studies, synthesis processes, and practical applications. This stoichiometry reveals different potential configurations (Komatsu, 2001), which lead to different stacking possibilities. For this reason, the prospects for formation of nanotubes are diverse and urgently require more theoretical studies. Graphitelike $\mathrm{C}_{3} \mathrm{~N}_{4}$ should encounter potential applications in organic semiconductors, and similar applications can be envisaged for $\mathrm{C}_{3} \mathrm{~N}_{4}$ nanotubes. There is little work reported on the synthesis of $\mathrm{C}_{3} \mathrm{~N}_{4}$-MWNTs (Cao et al., 2004), no experimental evidence of $\mathrm{C}_{3} \mathrm{~N}_{4}$-SWNTs has been given, and more theoretical studies are still expected.

\section{Boron nitride nanotubes}

Inspired by the stability of C-NTs, the parallel research field boron nitride nanotubes began. These structures were originally proposed based on tight-binding calculations by Rubio et al. (1994). In this study, the first indications of radical differences from their carbon counterparts were also suggested. Hexagonal boron nitride ( $h$-BN), like carbon, can be found with $s p^{2}$ and $s p^{3}$ configurations, and this led to the idea that $h$-BN might also form nanotubes (Zunger et al., 1976; Hamilton et al., 1993). Although the geometrical structures of $\mathrm{C}$ and $\mathrm{BN}$ nanotubes are similar, the electronic properties are radically different. As will be analyzed in later sections, C-NT can be metallic or semiconducting, whereas BN nanotubes are all stable wide-band-gap semiconductors
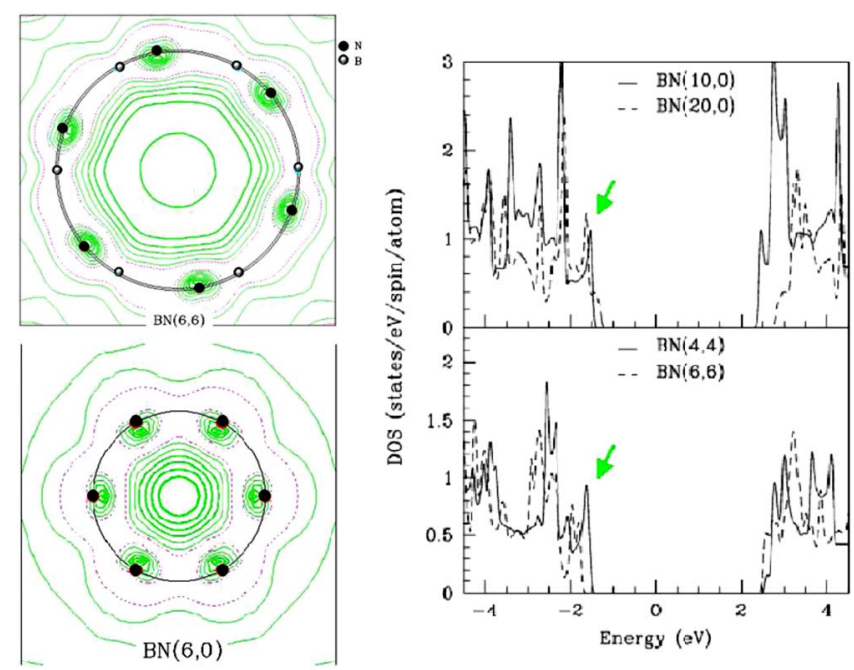

FIG. 9. (Color online) In the case of BN-NTs no metallic species are observed for the different chiralities. In the left side, contour plots of the charge density corresponding to the bottom of the conduction-band state of an armchair and a zigzag BN-NT are depicted. N atoms are indicated by solid circles and $\mathrm{B}$ atoms are represented by empty circles. These structures exhibit a uniform band gap. The DOSs of BN tubes with different chiralities are shown on the right. Image from Wirtz and Rubio, 2010, related to Blase et al., 1994.

independent of their helicity and diameter and whether the nanotube is SW or MW. Blase et al. (1994) calculated that strain energies are smaller for $\mathrm{BN}$ nanotubes than for carbon nanotubes of the same radius, owing to a buckling effect which stabilizes the BN tubular structure. They used a local-density-functional approximation, finding that for most of the BN-NT chiralities the lowest conduction band should be free-electron-like. This is summarized in Fig. 9, in which contour plots are shown (left) depicting the charge density corresponding to the bottom of the conduction band state of an armchair and a zigzag BN-SWNT. Blase et al. also predicted that for $(n, n) \mathrm{BN}$ nanotubes the bottom of the conduction band is located at around $4 \mathrm{eV}$ above the top of the valence band, independently of the tube diameter, and it is free-electron-like (constant charge density filling) in the inner part of the tubes. Self-energy corrections to the quasiparticle energies open the gap up to $\sim 5.5 \mathrm{eV}$.

In the contour plots of Fig. 9, $\mathrm{N}$ atoms are indicated and as well as B atoms. These structures exhibit a uniform band gap. No metallic BN tubes are observed for any chirality. On the graphs on the right of Fig. 9 the DOS of BN tubes with different chiralities are shown. The physical properties of BN nanotubes will be discussed in later sections. BN nanotubes are expected to have exceptional behavior in nano-optoelectronics ( $\mathrm{Ra}-$ dosavljević et al., 2003).

\section{SYNTHESIS OF HETERONANOTUBES}

Many methods are used in production of single-walled carbon nanotubes. The well-established techniques 
adopted by different research groups vary mainly as to the product desired. Nevertheless, the issues common to all synthesis methods, are still based on a partial understanding of the interdependence of the conditions optimal for growth regardless of the product. At present, the study of physical and chemical properties demands highquality material rather than mass production, but some applications do require large amounts of relatively clean material. There have been tremendous achievements in recent years in selective growth of pristine carbon nanotubes. At the beginning of this decade the CoMoCAT method using a bimetallic catalyst was able to produce samples rich in $(6,5)$ and $(7,5)$ chiralities (Kitiyanan et al., 2000; Bachilo et al., 2003). Recently the introduction of ultracentrifugation- and electrophoresis-based methods has made possible the separation of single-walled carbon nanotubes according to metalicity (Arnold et al., 2006; Yanagi et al., 2008; Tanaka et al., 2009). The synthesis of heteronanotubes of $\mathrm{C}, \mathrm{B}$, and $\mathrm{N}$, and their corresponding doped species has not developed side by side with the quality improvements for pristine C-NTs. Most experimental studies started only after the introduction of well-established C-NT production methods in the 1990s. Several of the well-known techniques applied for the synthesis of pristine C-NTs have been attempted, but most without immediately fruitful results. To develop the different reaction environment for growth of these different kinds of nanotube is not a straightforward task. This will be clarified throughout this section, with focus on the drawbacks encountered in each technique. In order to achieve a clear picture of the subject matter, the synthesis and use of MWNTs and SWNTs must be treated separately. Here we are oriented to single-walled nanotubes. Information about multiwalled structures can be found in different reviews and contributions (Terrones et al., 2004; Ewels and Glerup, 2005; Golberg et al., 2007; Ayala, Arenal, et al., 2010), which cover this topic thoroughly.

\section{A. Chemical vapor deposition}

Chemical vapor deposition (CVD) methods are routinely used for mass production of C-SWNTs, mainly with floating and supported catalysts. However, the production of heterostructures is still actively being explored, with less success. In principle, for the case of pristine C-NTs, CVD is a processes in which volatile precursors are used to provide a carbon feed source to a catalytic agent at elevated temperatures $\left(350-1000{ }^{\circ} \mathrm{C}\right)$, inducing the formation of elongated C-NTs. However, it is not a trivial matter to introduce nanotube wall modifications with $\mathrm{B}$ and $\mathrm{N}$ atoms through direct synthesis via CVD. The production of pristine C-NTs, which can reach several grams per batch, is in strong contrast with the production of structures such as $\mathrm{B}$ - and $\mathrm{N}$-doped C-NTs as well as heteronanotubes of $\mathrm{B}, \mathrm{N}$, and $\mathrm{C}$, in which case the material is still obtained in milligrams.

Most attempts to synthesize $\mathrm{N}$-doped nanotubes by CVD from various groups have resulted mainly in multiwalled structures that maintain a bamboolike morphol- ogy. As a common feature, in those experiments various hydrocarbons were mixed with different nitrogencontaining compounds such as melamine, acetonitrile, triazine, pyridine, and benzylamine, among others. Almost all these results came to the consensus that nitrogen-induced disorder in the walls of the nanotubes and inhibited the formation of single-walled tubes, which in turn also promoted the formation of bamboolike stacking. However, recent results on nitrogen-doped single-walled CNTs have suggested that this stacking can be directly related to the amount of catalyst and precursor introduced into the reaction zone. The extraction of the material from the reaction zone also seems to play an important role, in obtaining single-walled nanotubes with CVD techniques. Villalpando-Paez et al. (2006) reported the first single-walled nitrogen-doped nanotubes obtained by aerosol-assisted (AA) CVD employing a solution of ferrocene in different compositions of ethanol and benzylamine mixtures. They noticed that the products collected in the flow consisted of long strands of nitrogen-doped single-walled nanotubes, whereas the materials attached to the wall of the reaction tube were mainly bamboolike multiwalled nanotubes, as Terrones, Kamalakaran, et al. (2000) reported in previous work. The nitrogen content of these $\mathrm{CN}_{x} \mathrm{C}$-SWNTs was later found to be 0.3 at. \% (Elias et al., 2010). Similar N incorporation limits from benzylamine were reported for mixtures of single- and double-walled N-doped CNTs obtained in a traditional CVD system modified to attain high vacuum base pressure before the introduction of nondiluted $\mathrm{C}$ and $\mathrm{N}$ precursors (Ayala, Grüneis, Gemming, Grimm, et al., 2007). The analogous results for these materials obtained in two completely different systems are consistent and suggest that the maximum $\mathrm{N}$ atomic incorporation is ruled by the precursor chosen for synthesis. It was also possible to identify a $\mathrm{N}$ incorporation profile for samples rich in single- and doublewalled (DW) nanotubes (see Fig. 22). Similar experiments with acetonitrile illustrated how the morphology of the nanotube material is related to the composition of the reaction atmosphere (Ayala, Grüneis, Kramberger, et al., 2007). In $2005 \mathrm{Kim}$ et al. (2005) reported the use of bimetallic Fe-Mo catalysts supported in $\mathrm{MgO}$ to synthesize DWNTs, and later Panchakarla et al. (2007) used a similar method also applicable for nitrogen- and borondoped C-DWNTs. Liquid injection and water-plasma CVD have also been used to produce $\mathrm{CN}_{x}$-SWNTs. Keskar et al. (2005) were able to produce isolated $\mathrm{CN}_{x}$-SWNTs on bare quartz and $\mathrm{SiO}_{2}$-Si substrates with the former method, Min et al. (2007) deposited bundles on the same kind of substrates and reported the formation of high numbers of pyridinic sites; however, for such nanotube material a bulk probing method is not the most appropriate and local inspection should still be done. As mentioned, Panchakarla et al. (2007) succeeded in obtaining doped C-DWNTs. For the particular purpose of obtaining B-doped material, they decomposed $\mathrm{CH}_{4}$ and Ar mixtures in the presence of diborane. Although this work showed $\mathrm{x}$-ray photoemission spectroscopy (XPS) results to prove B concentration, a line- 

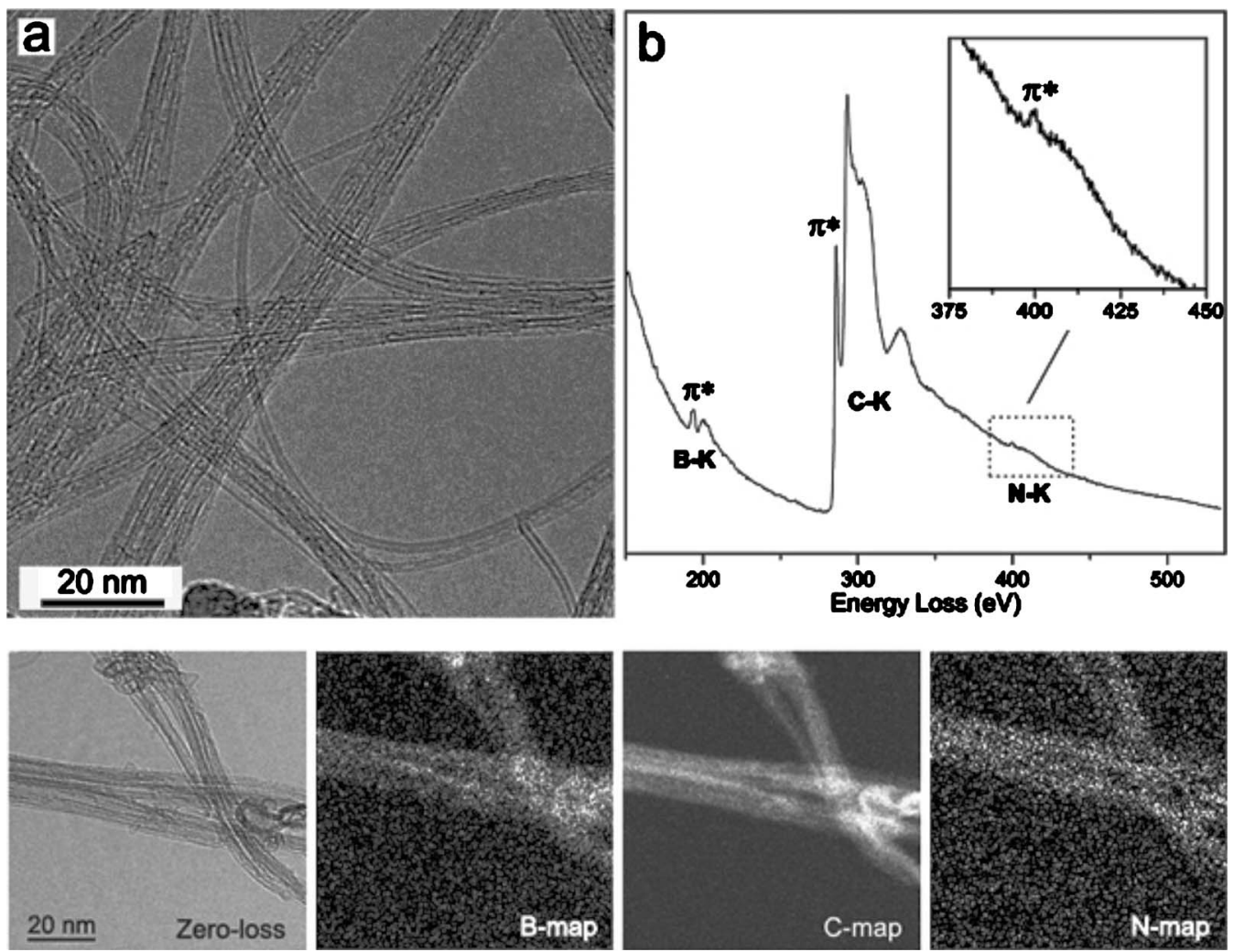

FIG. 10. Wang et al. (2006) reported a bias-assisted HF-CVD process to synthesize BCN-SWNTs in large scale. Upper panel: TEM bright-field image of bundles of BCN-SWNTs. Electron-energy loss as well as energy-filtered imaging were performed along the SWNT bundles, confirming the BNC structure. Adapted from Wang et al., 2006.

shape analysis of the broad spectrum of the B $1 s$ core signal is still required. Further studies are expected on doped C-DWNTs to distinguish the nanotube material from other by-products that contain B bonded to C. The synthesis of boron-doped C-SWNTs was recently reported (Ayala et al., 2008), using a nondiluted triisopropyl borate precursor and Fe-based supported catalyst in a CVD system working under controlled high-vacuum conditions. They found that, although high-quality C-SWNTs can be formed in a wide temperature range, $\mathrm{B}$ is not always incorporated in the carbon nanotube network. According to the synthesis temperature, the boron carbide and boron oxide concentrations can be more than 80 at. \% of the B content in a sample. In this case, less than 1 at. \% concentration can be attributed to the nanotube wall doping.

Chemical vapor deposition is a popular and very controlled method to produce C-SWNTs, but, as mentioned, the situation is radically different in the case of BN-NTs, which have been produced only in a limited manner in MW form. Recently Wang et al. (2006) reported a biasassisted HFCVD process to synthesize BCN-SWNTs on a large scale. They employed a bimetallic Fe-Mo and $\mathrm{MgO}$ catalyst in $\mathrm{CH}_{4}, \mathrm{~B}_{2} \mathrm{H}_{6}$, and ethylenediamine reaction atmosphere. TEM studies with analytic identification of B, N, and C were presented (see Fig. 10).

\section{B. Arc discharge}

The first composite sheets and multiwalled nanotubes containing carbon, boron, and nitrogen were grown with the arc discharge method employing graphite cathodes and amorphous boron-filled graphite anodes in a nitrogen atmosphere (Stephan and Ajayan, 1994). The traditional arc discharge method for the synthesis of CNTs is a high-temperature method based on the ignition of an arc between two graphite electrodes in a gaseous background (Iijima, 1991). The arcing evaporates the carbon and it condenses during the cooling process. It is well known that the derived product and the yields are mainly dependent on the atmosphere and catalysts utilized. In order to obtain nanotubes doped with $\mathrm{N}$ and $\mathrm{B}$, two variations have been applied. The first suggests the use of solid mixtures in the electrodes with compounds containing the doping agent desired (Glerup et al., 2004), whereas the second type of experiment uses pure graphite electrodes in a gaseous atmosphere (e.g., $\mathrm{NH}_{3}$ for the synthesis of $\mathrm{N}$-doped nanotubes) (Droppa et al., 2002). The arc discharge work by Glerup and co-workers differed from previous experiments for $\mathrm{CN}_{x}$-MWNT synthesis mainly in the target preparation. They proposed the introduction of nitrogen-rich precursors in the anode rods and reached doping levels up to 1 at. \% of $\mathrm{N}$ in C-SWNT in bundles [characterized by electron energyloss spectroscopy (EELS)]. These nanotubes are shown in Fig. 11. To the best of our knowledge, even though arc discharge has been used in attempts to produce B-doped C-MWNTs (Charlier et al., 2002) no experimental report of B-doped C-SWNTs is available in the literature. Although a higher incorporation of the dopant has been suggested upon higher concentrations (of $\mathrm{N}$ and $\mathrm{B}$ ) in 

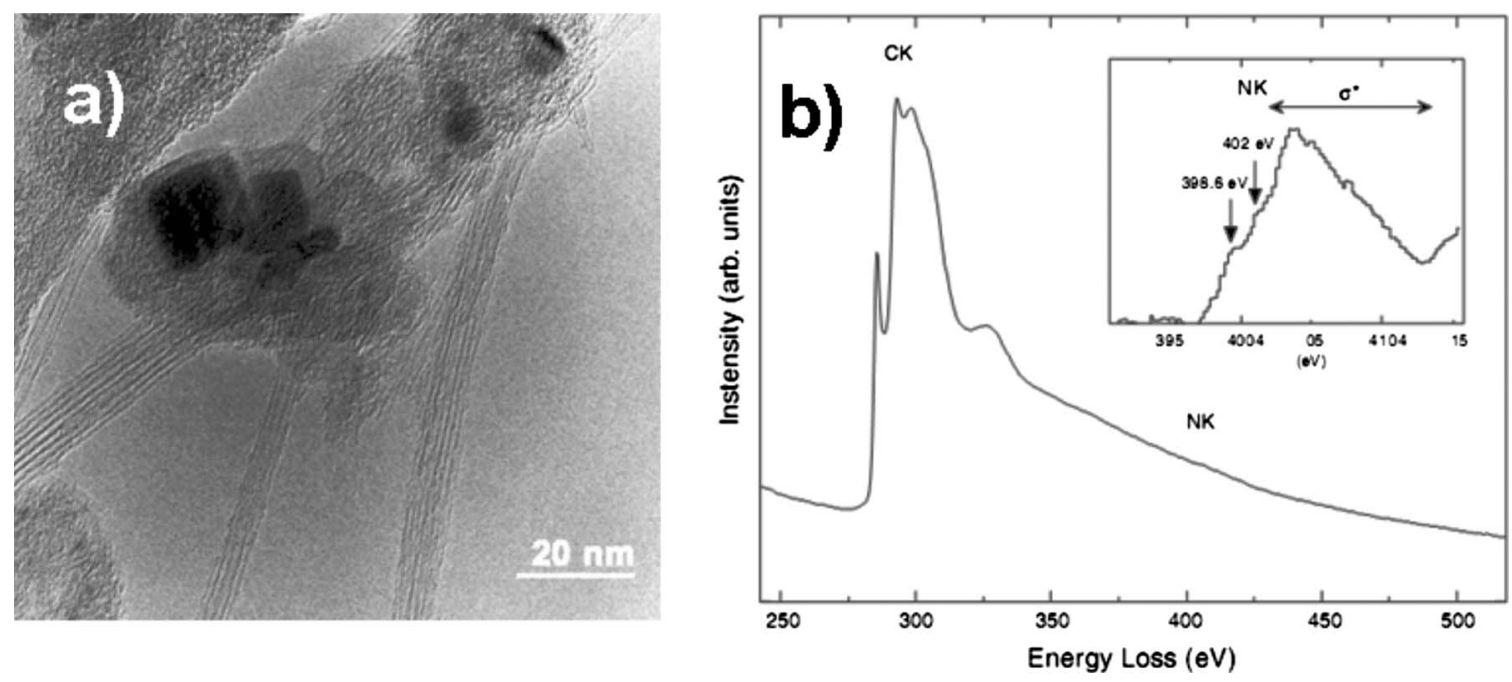

FIG. 11. One of the successful methods to synthesize $\mathrm{CN}_{x}-\mathrm{SWNTs}$ is arc discharge. The images show (a) TEM micrograph of the material produced with this technique. From Glerup et al., 2004. (b) EELS spectra with inset on the $\mathrm{N}$ edge from the same reference.

the electrodes, it still remains an open question if the incorporation of heteroatoms in the nanotube walls corresponds linearly to the electrode composition. The same concern applies for targets used in the laser ablation method and other techniques described later. For CVD methods, it has been shown that there is a limit related to the reaction atmosphere (Kim et al., 2005; Ayala, Grüneis, Gemming, Grimm, et al., 2007; Elias et al., 2010).

The first BN-MWNTs (Chopra et al., 1995) and BNSWNTs (Loiseau et al., 1996) were synthesized using an adapted arc discharge technique. By analogy to the use of graphite electrodes in C-NT synthesis, the natural choice would be the use of $h$-BN electrodes to obtain BN-NTs. However, $h$-BN has an insulating character, which was the main initial drawback. This difficulty was overcome in 1996 by arcing $\mathrm{HfB}_{2}$ electrodes in a $\mathrm{N}_{2}$ atmosphere (Loiseau et al., 1996). $\mathrm{BN}-\mathrm{HfB}_{2}$ is a metallic compound with a high melting point and known for its ability to react chemically at high temperature with $\mathrm{N}_{2}$. The morphology of these samples can be seen in the TEM studies and high-resolution micrographs in Fig. 12. By this procedure, a mixture of pure BN-SWNTs and MWNTs was produced, but in a relatively low yield. In further experiments using a plasma-arc method, Cumings and Zettl (2000) produced almost exclusively macroscopic amounts of pure and BN-DWNTs. In this case, nitrogen-free, boron-rich conducting electrodes incorporating a small amount of nickel and cobalt, as a catalyst, were employed in a pure nitrogen gas environment.

Recently an adaptation of the conventional arc discharge approach has been developed (Lee et al., 2006), which exhibits promising performance for the largescale production of $\mathrm{BN}$ nanotubes. This method employs the arc jet technique, also known as the plasma torch, which has the advantage of being a continuous process. Here a mixture of $h$-BN and catalytic powders (Ni and $\mathrm{Y}$ ) is used. The plasma formed in these experi- ments arises from a mixture of $\mathrm{Ar}$ and $\mathrm{N}_{2}$ at temperatures between 5000 and $20000 \mathrm{~K}$. So far the products have been shown to be a mixture of highly crystalline BN-SWNTs and BN-MWNTs.

\section{Laser ablation and vaporization}

Various techniques including CVD and arc discharge have been used to synthesize BN-NTs; however, laser ablation was the first method to successfully produce BN-NTs in bulk quantities (Yu et al., 1998; Lee et al., 2001; Arenal, Stephan, et al., 2007). The laser ablation technique uses either pulsed or continuous lasers to vaporize a target inside a furnace at high temperatures (Rümmeli et al., 2006; Arenal, Stephan, et al., 2007). The conventional graphite targets are usually mixed with a small amount of a transition metal catalyst in order to condense the SWNTs in the presence of different inert gas environments, which keep the reaction chamber at a

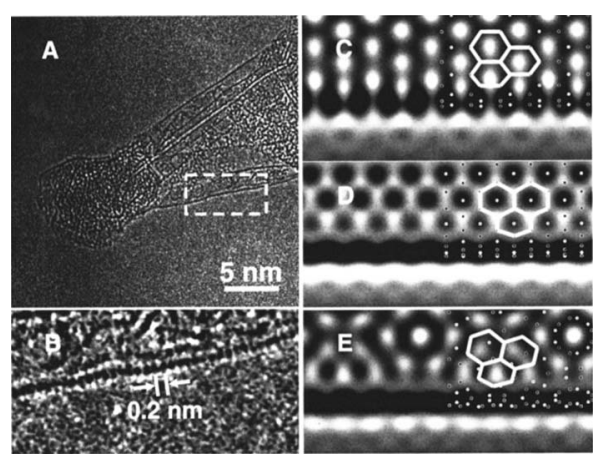

FIG. 12. BN-SWNTs obtained with a catalyst-free arc discharge synthesis method (a) HRTEM of BN-SWNTs emerging from a boron nanoparticle (Lee et al., 2001). (b)-(d) The transmission TEM images in high resolution are a helicity study of BN-SWNTs produced with this method. 


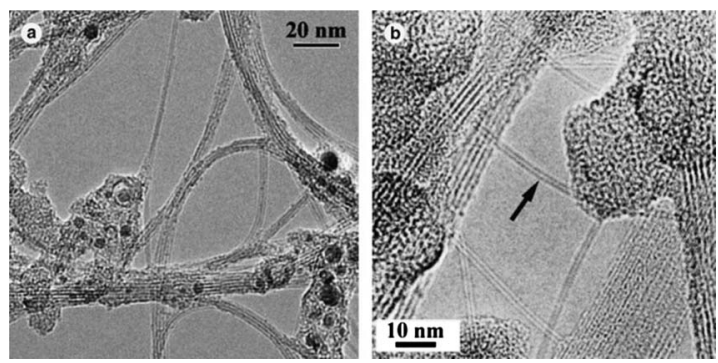

FIG. 13. Laser ablation B-doped nanotubes. The TEM images correspond to material produced from targets with nominal boron concentrations of (a) 0 and (b) 2.5 at. \%. From McGuire et al., 2005.

certain pressure level. In the case of BN-SWNTs produced by the adapted laser vaporization technique, the target is a mixture of $h$-BN microcrystallites (Golberg et al., 2003; Arenal, Stephan, et al., 2007), and the synthesis is carried out in a $\mathrm{N}_{2}$ atmosphere. In any case, as the laser ablates the target nanotubes are formed and are usually carried by the gas flow onto a cold finger. In this process, the parameters can be varied in order to obtain either doped C-SWNTs or heteronanotubes of B, N, and C.

In a similar manner to the preparation of electrodes for arc discharge, the ablated targets have been prepared with different kinds of compounds containing the dopant agents. Given the technological difficulties of detecting low dopant concentrations in nanotube samples, the first estimations of the $\mathrm{B}$ dopant concentration obtained by this method were directly related to the target mixtures (Gai et al., 2004; McGuire et al., 2005). Until recently laser ablation was the only technique known to produce B-doped C-SWNTs. The introduction of B into graphite-Co-Ni targets made possible the formation of single-walled nanotubes, whose overall morphology is shown in Fig. 13. The study by Gai et al. (2004) on this type of material suggested the difficulty of incorporating $\mathrm{B}$ in the graphitic network. They characterized the samples using HRTEM and EELS, and found SWNTs in the products when the $\mathrm{B}$ content in the target material was up to 3 at. \%. For higher B concentrations in the graphite target (3.5 at. \%), graphite and metalencapsulated particles were mainly generated, and only small quantities of SWNTs were obtained under these conditions. The evidence of substitutional B in these C-NTs was reported recently by Ayala, Reppert, et al. (2010).

The first N-doped SWNTs produced by laser vaporization were successfully synthesized only recently (Lin et al., 2008, 2009). The nitrogen content was studied by means of EELS in the TEM. They performed the first combined STS-TEM study of N-doped SWNTs (see Fig. 14); however, the identification of the $\mathrm{N}$ bonding environment was not possible from a spectral deconvolution of the $\mathrm{N}$ core level edge. Furthermore, Enouz et al. (2007) reported the possibility of using laser vaporization to produce BCN-SWNTs.

\section{Other methods}

\section{Ion implantation}

Xu et al. (2007) and Xu, Minniti, et al. (2008) proposed $\mathrm{N}$ ion implantation postgrowth in commercial nanotubes obtained by different techniques. These experiments are performed in a UHV chamber allowing in situ oxygen treatment, annealing, and latter implantation of lowvoltage $\mathrm{N}_{2}{ }^{+}$ions. Although an exhaustive study of the material postdoping has been made by XPS, with consistent line-shape analysis, a high percentage of $\mathrm{N}$ to $\mathrm{C}$ bonding in a tetrahedral $s p^{3}$ configuration was detected. This confirmed that the technique inevitably produces disorder in the C network. Further morphological studies are required to differentiate doped material from other carbonaceous amorphous by-products.

\section{Substitution reactions}

This technique, also known as the carbothermal process, was envisioned because of the structural similarities of the $h$-BN and graphite networks. The substitution reactions employ carbon nanotubes as template materials in whose presence a chemical reaction should occur. In fact, the carbothermal process is a modification of one of the most widely used synthesis methods to obtain $h$-BN: the reaction of boron oxide (or boric acid) with ammonia, at temperatures close to $900{ }^{\circ} \mathrm{C}$. In the case of nanotubes, this "substitution reaction" assumes a continuous atomic substitution between $\mathrm{C}$ and the heteroatoms (B and N) present in the reaction. Han et al. (1998) were the first to successfully achieve the production of $\mathrm{BCN}-\mathrm{MWNTs}$. These experiments were performed with mixtures of $\mathrm{B}_{2} \mathrm{O}_{3}$ and C-MWNTs at elevated temperatures $\left(1200-1700{ }^{\circ} \mathrm{C}\right.$ ) in $\mathrm{N}_{2}$ and $\mathrm{NH}_{3}$ atmospheres. Shortly after this, Golberg et al. (1999) reported the first results of BN-SWNTs produced with this method. A later study from the same group (Golberg, Bando, Burgeois, et al., 2000) reported a large scale synthesis and HRTEM analysis of B- and N-doped C-SWNT bundles where up to 10 at. $\%$ of $\mathrm{B}$ and 2 at. $\%$ of $\mathrm{N}$ were detected.

Later Borowiak-Palen et al. (2003) employed a substitution reaction similar to the one employed by Golberg, Bando, Burgeois, et al., 2000). They used bundles of C-SWNTs as templates and heated them in presence of boron oxide at $1150{ }^{\circ} \mathrm{C}$ while a flux of $\mathrm{NH}_{3}$ was continuously used as etching agent. They observed a high content of boron (between 10 and 15 at. \%), which in some cases was higher than the content reported by Golberg, Bando, Burgeois, et al., 2000). Such high B content apparently conflicted with the results for B solubility in C systems, which was later elucidated by Fuentes et al. (2004) in a combined theoretical and experimental study. They demonstrated that the stability of these B-containing C-SWNTs was justified by the actual formation of $\mathrm{BC}_{3}$-SWNTs. In this case it is not adequate to describe these nanotubes as boron-doped nanotubes. $\mathrm{BC}_{3}$ nanotubes are a completely different case, as explained previously. The high content of B detected in the 


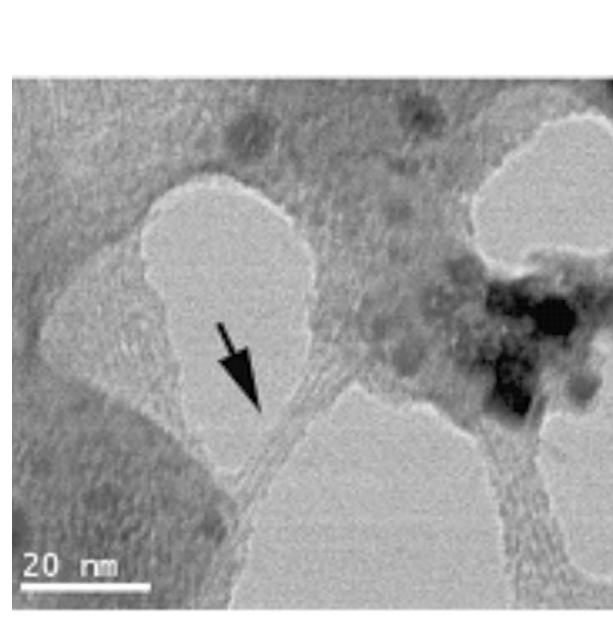

(a)

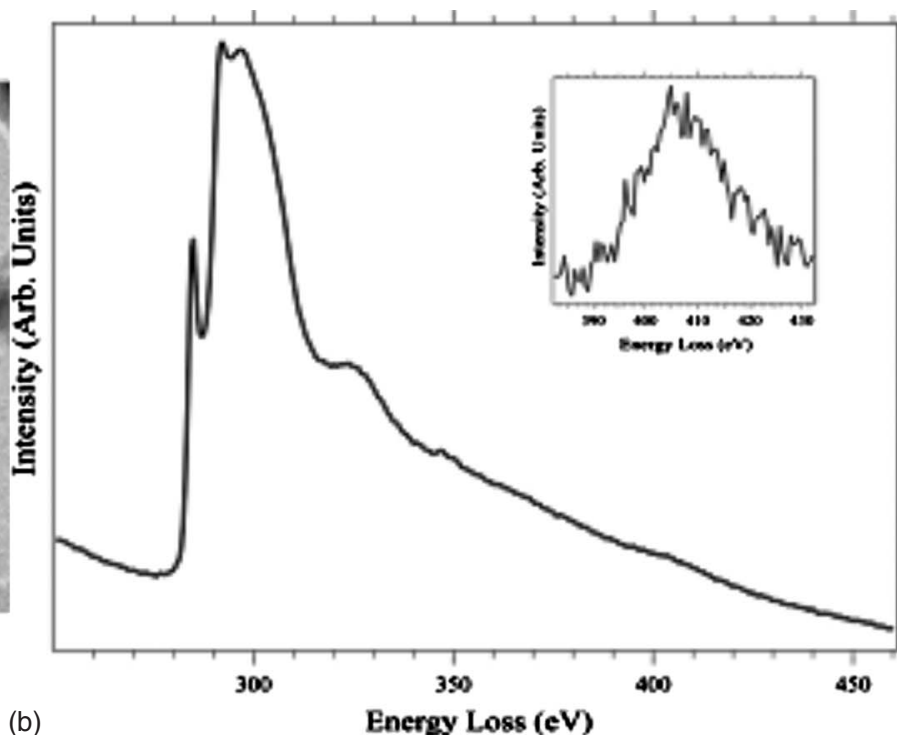

(b)
Energy Loss (eV)

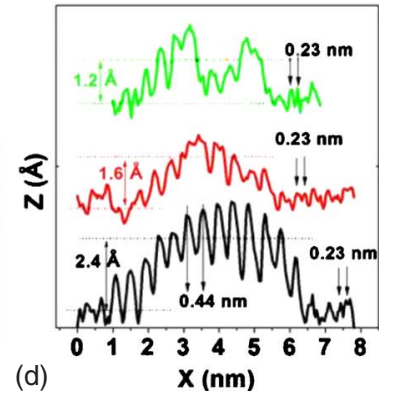

FIG. 14. (Color online) One of the recent methods to successfully produce $\mathrm{CN}_{x}-\mathrm{SWNTs}$ is the lasere vaporization technique. A TEM image is shown in (a) and the electron-energy-loss spectrum (b) recorded on the $\mathrm{CN}_{x}$-SWNT bundle is marked by an arrow in the bright-field image. The EELS spectrum displays the $\mathrm{C}$ and $\mathrm{N} K$ edges (emphasized in the inset). (c) STM images of a nanotube where the tunneling voltages are 900,800 , and $700 \mathrm{mV}$ from top to bottom, respectively. (d) The corresponding intensity profiles. From Lin et al., 2008.

nanotube network is a result of the formation of the predicted $\mathrm{BC}_{3}$ nanotubes. This explanation of the high content of boron in the lattice will be clarified in the sections on electronic and optical properties.

\section{Ball milling}

Although this method does not produce SW structures, it represents one optional way to synthesize $\mathrm{BN}$ MWNTs and fibers, and it also inspired other synthesis methods for BN-SWNTs. The first reported experiments by Chen, Chadderton, et al. (1999) and Chen, Gerald, et al. (1999) involved ball milling of elemental $\mathrm{B}$ in a $\mathrm{NH}_{3}$ flow. The products obtained were BN multiwalled nanotubes with a bamboolike morphology. Subsequent studies with variations in the reaction atmosphere led mainly to the formation of nanotubes with different diameters.

\section{CONTEMPORARY EXPERIMENTAL AND THEORETICAL METHODS FOR ACCESSING THE PHYSICS AND CHEMISTRY OF HETERONANOTUBES}

It is imperative to understand and control the effects of dopants in low-dimensional systems (as well as de- fects induced by dopant incorporation) if such structures are to be used for engineered materials and nanoscale devices. As mentioned, if we deal with the case of low dopant concentration, changes of structure and electronic properties are extremely sensitive to the use of the different experimental characterization tools and require the support of theoretical studies. The current status of this field has led to the general conclusion that a wide range of bulk and local probes have to be applied in order to unravel the details of the modification of the structural, electronic, and chemical properties. In fact, the use of techniques with high energy, momentum, and spatial resolution does not compete with local probing: "they should rather have a complementary use." The present section is devoted to a discussion of the potentials and limitations of the most commonly used techniques, whether spatially resolved or not, in order to access the physics and chemistry of the types of nanotubes described herein. Although the synthesis of doped C-SWNTs has been reported for several years, it is important to remember that their structures are still far from being fully understood. The complexity of analyzing samples with multiple morphologies demands a deep understanding of the intrinsic properties of the materials 
examined. It is essential to understand, characterize, and ultimately control the bulk or local stoichiometry based on the final application expected for a specific type of heteronanotube.

To encompass the full methodology, theoretical studies are required to predict the physical behavior of these molecular nanostructures. In this respect, the proper description of mechanical and electronic properties of nanotubes, as well as their growth mechanisms, requires consideration of a large number of atoms and long time scales and consequently sophisticated techniques. For this reason, the end of this section comments on the methods commonly adopted for large-scale calculations of the electronic properties of molecular nanostructures. This intends to clarify how they have been adapted from the theories available for materials at macroscopic, mesoscopic, and nanoscopic scales.

\section{A. Spatially resolved techniques}

Several local probing methods are commonly used for the identification and characterization of heteronanotubes. The first is TEM, which has historically been widely employed to study the morphology and atomic structure of nano-objects. In recent years, several spatially resolved techniques have been incorporated and improved in the TEM, converting this instrument into a powerful analytic tool. Scanning-probe-microscopybased techniques have also been further developed, allowing atomic resolution and mapping of the electronic structure of the underlying nanotube geometry.

\section{TEM bright-field conventional and high-resolution imaging}

With TEM, the basic morphology of the tubes can be found in a simple way. For instance, in a high-resolution image of a SWNT one always observes prominent parallel (002) lattice fringes, which are views of the tube walls that remain tangent to the electron beam. This happens for all tube orientations since at all positions there are some parts of the tube wall that satisfy the Bragg condition. However, the lattice structure corresponding to the $(h k 0)$ and $(h 0 l)$ lattice planes can also be imaged to spot patterns showing hexagonal symmetry. This was attempted in early observations, but the imaging of atomic positions has only recently been possible with the introduction of microscopes equipped with spherical aberration correctors (Smith, 2008).

Historically image resolution has been restricted by the unavoidable spherical aberration of the objective lens, but aberration correction has been achieved in both fixed-beam TEM (Haider et al., 1998) and scanning TEM (Krivanek et al., 1999). In recent years, there has been significant activity in this area. For fixed-beam microsocopes, aberration correction can be achived through either direct [insertion of multipole elements (hardware)] or indirect methods [holography and software approaches (exit-wave reconstruction methods)]. Uncorrected microscopes can provide a point resolution of $15-0.2 \mathrm{~nm}$, but for corrected TEM below $0.1 \mathrm{~nm}$ res- olution can be reached. The nanotube diameter can be directly measured from the fringe spacing with an accuracy of $\pm 0.5 \mathrm{~nm}$ at the Scherzer defocus [in practice, to maximize the transfer of information about the specimen, high-resolution images should normally be recorded in these conditions (Scherzer, 1949)]. However, for the nanotubes described here, no matter what kinds of nanotubes are observed, and even if the optimal alignment and focus conditions are used in the microscope, the image contrasts are not sensitive to the different nature of substitutional heteroatoms such as boron or nitrogen, since they also behave as weak-phase objects and display a phase contrast close to that of carbon.

\section{Analytic transmission electron microscopy}

TEM is not only a versatile technique for interpretation of the basic morphology of the nanotubes. Most commercial state-of-the-art microscopes can be obtained with various tools, which can access the electronic properties of the nanotubes. The analytical possibilities in a TEM encompass electron energy-loss spectroscopy (Egerton, 1996), energy-filtered TEM (EFTEM), and energy-dispersive x-ray spectroscopy (EDX), which also represents a powerful approach to investigate the structure, chemical composition, and properties of nanomaterials in a combined way. In principle, both EFTEM and EELS probe the same three-dimensional data space $I(E, x, y)$ containing both spectral $I(E)$ and spatial $I(x, y)$ information. Two kinds of information can be extracted from the EELS spectra depending on the energy-loss range investigated. The core loss region gives access to the chemical and electronic structure of the material, whereas the low energy-loss region $(\sim 50 \mathrm{eV})$, where interband and plasmon excitations are involved, provides information on its dielectric, optical, and electronic properties. The optimal way to obtain high spatial resolution, while performing analytical imaging, is to use a dedicated STEM instrument. In this case the spatial resolution of TEM EELS is mainly limited by the size of the probe, which is around $0.5 \mathrm{~nm}$. With the proper instrumental and illumination conditions one can extract two-dimensional (2D) elemental maps (1D elemental profile) corresponding to the different elements present in the sample with much improved spatial correlation (Muller et al., 2008). Arenal, de la Pena, et al. (2008) also showed that $2 \mathrm{D}$ chemical bonding maps can be extracted at the nanometric scale.

One of the main problems when working with small structures such as SWNTs is sample damage due to its sensitivity to the focused electron beam. The EFTEM mode is an alternative method for recording elemental maps, based on EELS. In both cases the energy-loss processes are identical. The difference between them is in how the information is acquired. In EFTEM an elemental map is directly recorded by selecting energy windows in ranges corresponding to the elemental core edges. If different elements are present in the sample, elemental maps are captured serially. 


\section{Scanning probe microscopy}

High-resolution TEM allows for a quick view with atomic resolution. However, scanning probe techniques such as atomic force microscopy (AFM) and its related variations as well as STM and STS allow one to get atomic resolution and to map the electronic structure of the nanotube geometry. Scanning tunneling spectroscopy has been used to determine the morphology and the local electronic structure of carbon nanotubes (Biró et al., 1998; Kim et al., 2000; Orlikowski et al., 2000; Ichimura et al., 2002; Odom et al., 2002; Hassanien et al., 2004). A typical example is the correlation between the energy gap in a semiconducting CNT and the tube diameter (Tans and Dekker, 2000). In fact, great advances in simulation of STM imaging for carbon nanotubes $(\mathrm{Ru}-$ bio, 1999; Lambin et al., 2003) also represent a great advantage in the inspection of wall imperfections and their effects on transport properties (Orlikowski et al., 2000). Some interesting results have been obtained for functionalized C-SWNTs (Kelly et al., 1999) but much less is known for $p$ - and $n$-substituted C-SWNTs and heteronanotubes. This might be mainly related to the technical challenges of localizing the doped tubes. In the case of $\mathrm{BN}$ nanotubes the issues are mainly related to measurements resulting in a giant Stark effect (Ishigami et al., 2005).

With these tools the presence of local defects or atoms can be identified by tracking changes in the local density of states. A recent report on $\mathrm{CN}_{x}-\mathrm{SWNTs}$ has shown STM-STS measurements (Lin et al., 2008, 2009) suggesting the presence of topological defects on the SWNTs as well as two localized states in the gap in the defect topological area. However, further studies are needed to correlate the presence of the identified defects with the incorporation of $\mathrm{N}$ atoms in the nanotubes.

\section{B. Momentum- and/or energy-resolved techniques}

In optical spectroscopy, the spatial resolution is lost. However, alternative information about the band structure and the vibrational properties of the constituents can be gained. Optical absorption spectroscopy probes the electronic band structure by direct excitation of an electron from the valence to the conduction band. Since $\mathrm{BN}$ tubes have a wide band gap, either multiphoton processes or uv light are necessary for this process to occur in $\mathrm{BN}$ nanotubes.

\section{Bulk-sensitive EELS}

An introduction to EELS in TEM was given in the section related to spatially resolved techniques. In this section we need to keep in mind only that, if a sample is irradiated with a beam of electrons, the energy losses caused by specific inelastic interactions can be measured; this is the basic principle of EELS spectroscopy. Nevertheless, TEM is not the only device coupled to such a system. In fact, EELS can also be performed in a purpose-built high-resolution spectrometer, which combines both good energy and good momentum resolution
(Fink, 1989). Unlike many electron spectroscopies, this method is volume sensitive. An energy and momentum resolution of $80 \mathrm{meV}$ and $0.05 \AA^{-1}$ (valence band excitations) and of $160 \mathrm{meV}$ and $0.06 \AA^{-1}$ (core excitations) can be achieved. Measurements of functionalized nanotubes utilizing films have been performed successfully with this method. Here the electronic structure of films as thin as $1 \mathrm{~nm}$ can be measured in transmission using high-resolution EELS. For such measurements, it is necessary to take into account the following preconditions: (1) high-energy electrons of $\sim 170 \mathrm{keV}$ and (2) an effective thickness of the free-standing samples of about $1000 \AA$. A more detailed description of the operation and principles behind these energy- and momentumresolved EELS experiments can be found in the review article by Fink (1989).

The quantity measured in EELS is the loss function, which is given by $\operatorname{Im}[-1 / \epsilon(q, \omega)]$. This provides information on the collective excitations of the electronic system (the plasmons). For energy losses below $\sim 50 \mathrm{eV}$, the loss function essentially describes plasmon excitations that arise from intraband and interband transitions. A Kramers-Kronig analysis (KKA) is then required to extract the real $\left[\epsilon_{1}(q, \omega)\right]$ and imaginary $\left[\epsilon_{2}(q, \omega)\right]$ parts of the dielectric function.

In contrast to optical spectroscopy, which probes only dipole-allowed $q=0$ transitions, one gets access here to the momentum dependence of the dielectric function. Consequently, nonvertical transitions can also be excited. HREELS allows one to probe important quantities such as the character of excitonic excitations, the spatial extension of excitons, and the degree of localization of electronic interband and intraband excitations. This technique is especially applicable to mesoscopic systems with an anisotropic electron density. The nanotubes reviewed here are among these systems and are an ideal matter of study with this technique, but it is necessary to include local field effects (Onida et al., 2002) for a correct approximation. The reader interested in going deeper into this topic should see Marinopoulos et al. (2002) and Kramberger et al. (2008), who have made detailed studies for graphite and C-SWNTs, respectively.

For illustrative purposes, an example of the dispersion of the optical interband excitations in an anisotropic system such as a BN-MWNT (Fuentes et al., 2003) is shown in Fig. 15. From a KKA, the momentum-dependent real and imaginary dielectric functions of this anisotropic system can be derived (Fuentes et al., 2003).

At higher energies, the loss function describes transitions from core or shallow core levels to unoccupied states derived from orbitals. These core excitations are governed by the dipole selection rule. In this respect, this method is complementary to the TEM-EELS measurements described earlier. The main difference is that the high energy and momentum resolution allow accessing the fine structure in the bonding environment as well as the modification in the conduction band, taking into account the effect of the core hole, with much more detail (resolution). One example is given in Fig. 16, where the $\mathrm{C}$ edges of different doped C-NTs are shown. For 


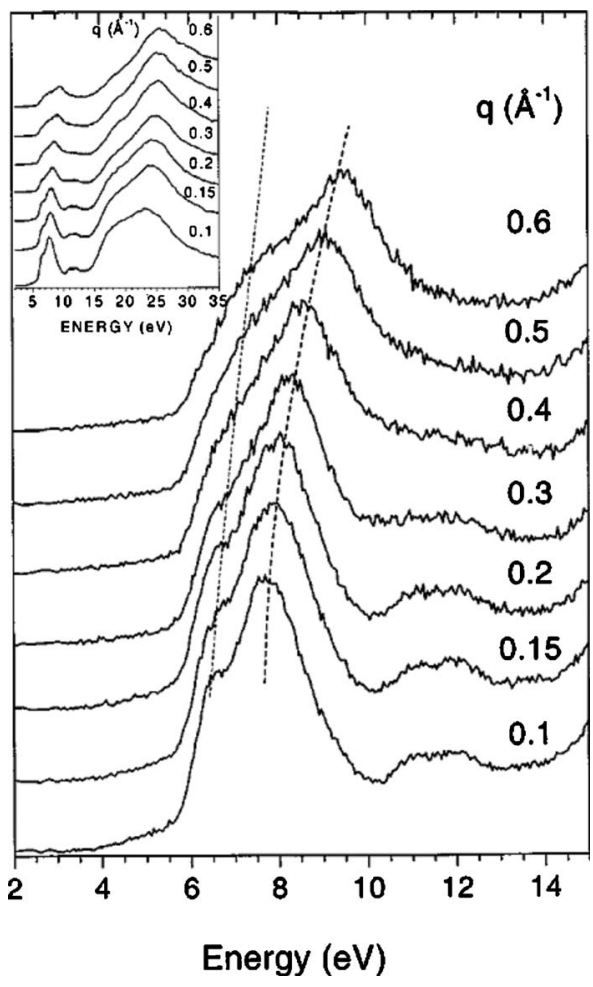

FIG. 15. Loss spectra of the multiwall $\mathrm{BN}$ nanotubes between 2 and $15 \mathrm{eV}$ as a function of the momentum transfer $q$, as measured by EELS. Inset: Wide energy range between 0 and $30 \mathrm{eV}$. From Fuentes et al., 2003.

$p$-type doping a clear change in the matrix element DOS of the former valence band yields an additional shoulder at low energy values, whereas for $n$-type doping the integrated intensity is decreased. This shows the changes in the charge transfer and bonding environments at the carbon nanotube sites.

\section{X-ray absorption and photoemission}

$\mathrm{X}$-ray absorption spectroscopy is a tool for analyzing core level excitations. This method relies on tunable
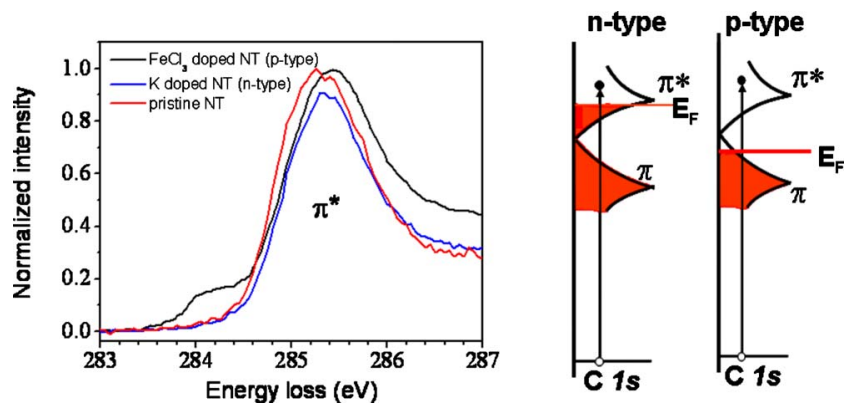

FIG. 16. (Color online) The matrix element weighted conduction-band response of C-SWNTs as a function of doping and the corresponding shift is analyzed by EELS on the example of the $\mathrm{C}$ edge of pristine nanotubes and bundles of nanotubes with $\mathrm{K}$ for $n$-type doping, as well as $\mathrm{FeCl}_{3}$ for $p$-type doping. The changes in the DOS upon doping for the $\mathrm{C}$ $1 s$ core level excitation process within the rigid band model are highlighted in the sketches. $\mathrm{x}$-ray sources at synchrotron radiation centers and is applied mostly in the total (partial) electron yield and the fluorescence yield modes. In principle, it is capable of providing the same information as the spatial- and angle-resolved core level EELS, but it has the advantage of having, in combination with third-generation light sources, the outmost energy resolution of up to $10 \mathrm{meV}$ at the $\mathrm{C} K$ edge. Therefore this method has been successfully applied to unravel the vHs in the conduction band of a C-SWNT (Kramberger et al., 2007; Ayala, Miyata, et al., 2009).

The second technique considered in this category is photoemission spectroscopy, which embraces one of the most common and successful techniques for identification of bonding environments. It is based on the wellunderstood photoelectric effect. Again, it can be divided into two energy scales. For the valence band region, the DOS and, in the case of angle-resolved photoemission (ARPES), the electronic band structure are analyzed. After the self-energy corrections are unraveled, basic correlation effects such as electron-phonon coupling can be analyzed. With state-of-the-art light sources and detectors, this technique is a powerful tool to determine the electronic structure of 2D systems such as graphene and graphite. For pristine and doped C-SWNTs, the lack of single-crystal samples explains why mostly angleintegrated photoemission has been applied so far. In this manner, some studies have been performed, unraveling the nature of the metallic Tomonaga-Luttinger liquid ground state in isolated metallic C-SWNT (Ishii et al., 2003) as well as the doping-induced change of an interacting metallic C-SWNT to a normal Fermi liquid (Rauf et al., 2004). However, we emphasize again that the samples for studies using valence band photoemission need very high purity, which might also explain the lack of results on substitutionally doped C-NTs.

In addition, for core holes, the various ionization processes have different probabilities. There is a different cross section for each final state. Because each element has a unique set of binding energies, XPS can be used to identify and determine the concentration of the elements. Variations in the elemental binding energies (chemical shifts) arise from differences in the chemical potential and polarizability of compounds and this, in combination with the high-resolution and sensitivity of state-of-the-art detectors and light sources, is particularly useful in the analysis of pristine (Kramberger et al., 2007; Ayala, Miyata, et al., 2009) and doped nanotubes with low doping amounts in particular (Ayala, Freire, et al., 2007; Ayala, Grüneis, Gemming, Büchner, et al., 2007; Ayala, Grüneis, Kramberger, et al., 2007). If the initial sample purity is checked by an XPS survey, this technique allows detection of less than $0.2 \%$ of dopants in different chemical environments (Kim et al., 2005). As mentioned later (in Sec. V) for nitrogen-doped nanotubes it is possible to detect, for instance, even $0.1-0.2 \%$ of $\mathrm{N}$ in a $\mathrm{C}-\mathrm{NT}$ and to determine the exact $\mathrm{N}$ bonding environment by use of a line-shape analysis (Ayala, Grüneis, Gemming, Grimm, et al., 2007). Elias et al. (2010) 
have also reported $\mathrm{N}$ incorporation in similar proportions.

\section{Optical techniques: Optical absorption, Raman, and luminescence spectroscopy}

Optical absorption spectroscopy is a natural characterization path that allows extraction of different pieces of information about the quality of a nanotube sample. For instance, the purity of a sample can be determined from the optical absorption data, since the area under the first interband transition peak of the C-SWNT in the optical absorption spectrum is a direct measure of the abundance of the C-SWNT in the sample. In fact, an optical absorption spectrum provides the optical transitions occurring between the vHs of the valence and conduction bands in the DOS, which depend closely on their metallic or semiconducting character and on their diameter.

In the case of pure C-SWNTs, their applications are especially focused in the infrared (ir) region, where they exhibit a saturable absorption effect. However, because of the large band gap of $h-\mathrm{BN}(>6 \mathrm{eV})$, optical absorption starts only in the ultraviolet regime. Thus, optical absorption spectra of $\mathrm{BN}$ tubes are difficult to measure and this may not be the method of choice for the characterization. Nevertheless, a theoretical understanding is of utmost importance as a first step toward the understanding of luminescence in BN tubes. The absorption spectra are conceptually interesting because they are dominated by a strongly bound exciton.

In the case of heteronanotubes, although little has been reported on the optical absorption spectra of $\mathrm{CN}_{x}$-SWNTs and $\mathrm{CB}_{y}$-SWNTs, interesting features have been seen, such as an acceptorlike band for the second case Borowiak-Palen et al. (2004). These results will be explained in detail in Sec. VIII.

In addition, photoselective resonance Raman spectroscopy and luminescence spectroscopy have been shown to be among the most powerful tools for analysis of C-SWNTs (Jorio et al., 2001; Bachilo et al., 2003). The strong characteristic Raman response of C-SWNTs as well as the strong near-infrared luminescence of individual semiconducting C-SWNTs makes them fast and reliable tools to asses the structural, vibrational, and electronic properties. Defects are easily detected in Raman spectroscopy because they locally break the symmetry of the crystal and relax momentum conservation rules that govern Raman scattering processes. Phonons with wave vector $q \neq 0$ can therefore become Raman active in the presence of defects via a double-resonance process. The phonon dispersions for graphene and $h$-BN are shown in Fig. 17.

The strongest disorder-induced feature in nanotubes is the so-called $D$ band at around $1300 \mathrm{~cm}^{-1}$. This band corresponds to an in-plane transverse optical phonon at the $K$ point, which is one of the high-symmetry points in reciprocal space, located at the zone boundary. The intensity of the $D$ band as compared with the Ramanallowed transitions is often used to estimate defect den-

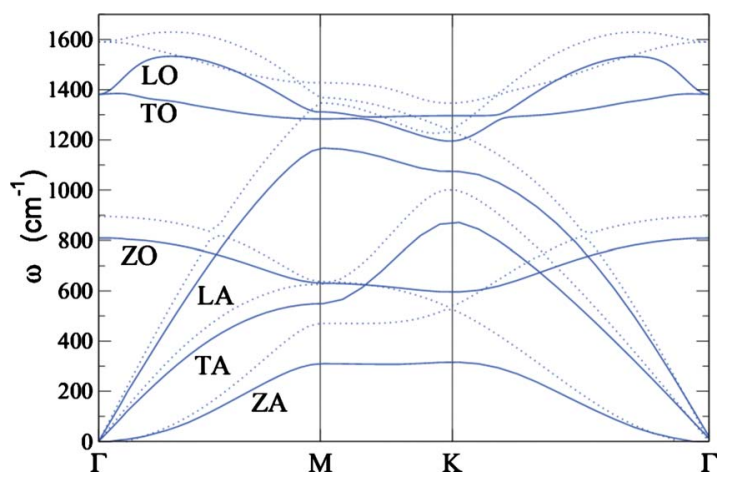

FIG. 17. (Color online) Comparison of the calculated phonon dispersion of (dashed) graphene and (solid) $h$-BN. From Wirtz and Rubio, 2010.

sities. It is known that the second-order process, in which two $D$-band phonons with opposite momentum are simultaneously emitted (Thomsen and Reich, 2000), is Raman allowed in pristine nanotubes. Furthermore, this mode and the $2 D$ or $G$ phonon mode of metallic C-SWNTs is characterized by Kohn anomalies (KAs). The doping-induced shift of the Fermi energy level is expected to modify the KAs. Calculations by Caudal et al. (2007) resulted in a model to determine the doping level from the value of the KA-induced frequency shift and from the linewidth of the $G$ peak. They also determined that the KA should be observed in the $D, 2 D$, and $2 G$ peaks. In addition, a detailed analysis of the doping-induced shifts of the $G$ peak, which is the strongest peak around $1590 \mathrm{~cm}^{-1}$, has been performed at high doping in the framework of the $\mathrm{C}-\mathrm{C}$ bond expansion and contraction within a rigid band model (Pichler et al., 2003; Chen et al., 2005). Positive charging should always cause an upshift in the $G$-band. This is because of the cumulative effect of the phonon renormalization and the change in the spring force constant of the C-C bond; for negative doping the two phenomena are competing with each other. The resulting behavior depends on the contribution of each effect. At low doping, this dopinginduced modification in the KAs has been used to explain the experimentally observed hardening of the $G$-line upon $n$-type doping (Caudal et al., 2007). This observation on the intercalation of bundled nanotubes has recently been confirmed by Kalbac et al. (2008) using electrochemical doping of individual C-SWNTs. A similar result has been observed for gated individual nanotubes (Tsang et al., 2007). For B- and N-doped C-SWNTs as well as for BN-SWNTs much less has been reported. The results of Maciel et al. (2008) cover mainly the $2 D$ peak in $p$ - and $n$-doped nanotubes. They find a splitting of the $2 D$ peak at higher or lower energy values in the Raman shift according to the nanotube charge state (see Fig. 18). Additional results have been reported for the shift of the $G$ line by McGuire et al. (2005). For singlewall BN-NTs (Arenal, Stephan, et al., 2007) offresonance Raman results have been discussed. These results will also be elucidated in detail in Sec. VIII. 


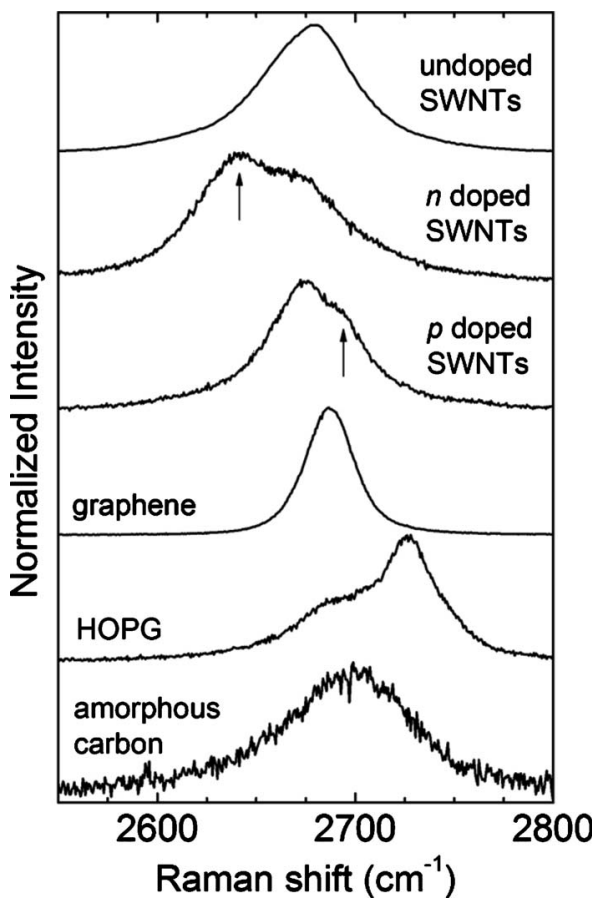

FIG. 18. Shifts and splitting in the $G^{\prime}$ response for different $s p^{2}$ carbon systems. From Maciel et al., 2008.

\section{Structural analytic techniques}

\section{X-ray diffraction}

This technique is probably one of the simplest ones to identify the presence of nanotubes in a sample (Saito et al., 1993). The average tube diameter and diameter distribution, the bundle thickness, and intertube spaces can be identified with x-ray diffraction (XRD) (Miyata et al., 2008). Although the amount of information is limited in the case of the peculiarities we are studying, an XRD pattern is sensitive to molecular or atomic adsorption on SWNTs (Maniwa et al., 2007) and this can be useful when analyzing potential applications.

\section{Electron diffraction}

In addition to imaging in bright-field conditions, one advantage of most TEM instruments is the possibility of recording electron diffraction (ED) patterns focused on individual structures. In principle, the structure of any SWNT can be described by either a set of two chiral indices $(n, m)$ or giving both its diameter $d$ and one helicity. High-resolution images can be recorded with the TEM, but this technique is sensitive to the orientation of the tube with respect to the electron beam and to the defocus conditions. The use of ED does not present these limiting factors; therefore it represents a reliable technique for obtaining the nanotube atomic arrangement. As a result, the diameter and chiral angle can be obtained, and in some cases it is also possible to deduce the $(n, m)$ indices, which fully identify any given NT.

Most techniques that are routinely used for determining diameters and helicity in C-NTs, such as Raman or scanning tunneling microscopy, are not applicable to all

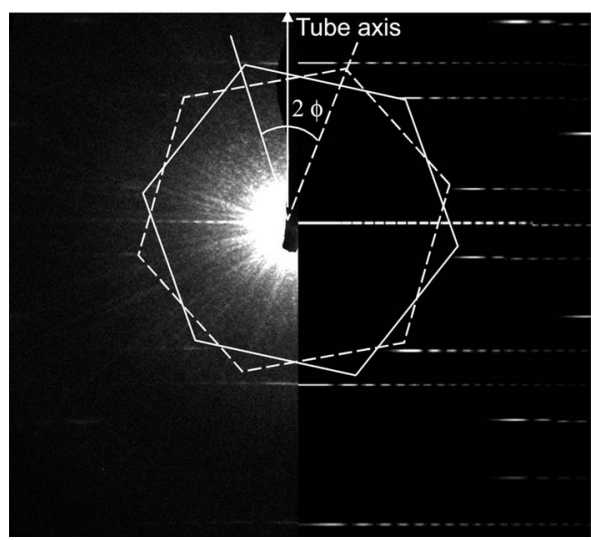

FIG. 19. Experimental and simulated ED patterns of a BNSWNT. The intensity profiles of the equatorial line of the experimental and simulated ED patterns of the tube are also represented. Adapted from Arenal, Kociak, et al., 2006.

types of graphitelike tubular structures. A clear example of this is BN-NT because of its wide band gap. ED does not have this limitation because it is based on the diffraction of electrons produced by organized geometrical atomic arrangements. Nowadays, ED has started to be more frequently used to identify diameters and chiralities of nanotubes. Complete descriptions of this technique can be found in Arenal, Kociak, et al. (2006); Qin (2006, 2007); Meyer et al. (2007).

To interpret the ED pattern of a nanotube, one can ideally consider a single-walled nanotube formed by wrapping up a two-dimensional hexagonal network of carbon or $\mathrm{BN}$ sheet along a chosen tubule axis. Electron diffraction studies of NTs using a TEM could be developed via two different techniques: selected-area electron diffraction (SAED or SAD) and parallel-beam nanobeam electron diffraction (NBED). The main advantage of NBED is a specimen area reduced below $50 \mathrm{~nm}$, which is useful (even necessary) for work on individual nanotubes. A SWNT can be considered as two parallel graphitelike sheets rotated with respect to each other by $2^{\circ}$ (the chiral angle); thus the ED pattern is the superposition of two rotated trigonal networks. This explains why the first intensity maxima in a SWNT roughly form two hexagons, as shown in the ED pattern of a BNSWNT in Fig. 19. For further analysis, it is more interesting to consider that the intensity is discretely distributed on lines (known as "layer lines"). The central one is called the equatorial line. The chiral angle of the nanotube is determined by measurement of the interspacing between the different layer lines. Arenal, Kociak, et al. (2006) found a high percentage of BN-SWNTs with zigzag chirality. In the same contribution they studied BNDWNTs, and observed that the value of the intertube distance and the significant difference of the chiral angle between the two tubes indicated lower interaction between the BN sheets than in bulk $h$-BN.

Golberg, Bando, Burgeois, et al. (2000) and Golberg, Bando, Kurashima, and Sato (2000) also performed high-resolution imaging and ED on B- and N-doped 
C-SWNTs in order to perform a population analysis of the helicities in such samples.

In any case, it is a particular challenge to work with individual nanotubes because the sections used for ED contain only a small number of identical atoms, which interact weakly with the electron beam, in comparison to other nanostructured systems. However, we recall that the introduction of heteroatoms can induce preferential orientation of the graphitelike tubular shells; this would be an ideal case to study with this technique, which has not yet been fully developed in the cases described in this review.

\section{Transport}

The electronic and transport properties of SWNTs involve many considerations, such as charge carrier localization, injection barriers, and diffusive and ballistic regimes. The conduction regime and transport magnitude are the main focus of attention in related research. In fact, the metallic or semiconducting character of a SWNT is directly inferred from its topological structure, but additional variables such as defects can induce diverse physical consequences (Charlier et al., 2007). Topological defects and tube-tube interactions represent the largest portion of the related theoretical research. However, the presence of foreign atoms introduces new interesting features in each case. The diversity of the structures treated in this review makes a rich playground of this topic.

Although publications are dominated by theoretical calculations, some experimental results for MWNTs are available (Choi et al., 2003; Krstić et al., 2003; Avriller et al., 2007; Zhang et al., 2008). Reports on experiments with SWNTs are few so far. However, some work has recently appeared, such as the experiment by $\mathrm{Xu}, \mathrm{Lu}$, et al. (2008), who suggested the possibility of converting metallic C-SWNTs into semiconductors by B and N codoping. They probed this by, making field-effect transistors devices, and later performed ab initio calculations of the structures with the doping levels obtained experimentally (between 3 and 8 at. \% considering a combined $\mathrm{B}$ and $\mathrm{N}$ total dopant concentration). To cite another example, Xiao et al. (2006) reported on the electrical transport properties of a Schottky junction formed by a pristine C-SWNT and a $\mathrm{CN}_{x}$-SWNT. They suggested that the chemical defects due to $\mathrm{N}$ doping induce a highly defective trap state, which strongly influences the charge transport along the whole structure.

In the specific case of low incorporation $(<0.5 \%)$ of boron and nitrogen in C-SWNTs, Latil et al. (2004) reported a drastic modification of the electronic properties at such doping levels. Das et al. (2007) proposed the use of Raman spectroscopy and transport measurements together to identify the doping level in C-SWNTs.

Recently Murata et al. (2008) reported the observation of the Meissner effect at $T_{c}=12 \mathrm{~K}$ in thin films of $\mathrm{CB}_{y}$-SWNTs synthesized with the laser vaporization technique. They pointed out that the Meissner effect was sensitive to the degree of uniformity of the thin film and that by optimizing the control of the deposition of the films higher $T_{c}$ values (between 30 and $40 \mathrm{~K}$ ) could be reached.

These are only few examples of the importance of transport studies in assessment of the physics and chemistry of heteronanotubes and doped C-SWNTs. The electronic and transport properties, which constitute the scope of the present review, are certainly among the most significant physical properties and crucially depend on the diameter, chirality, and atomic configuration. This section has introduced only the importance of the transport properties but a broader discussion related to the theoretical aspects of transport studies is found in Sec. VII, with a compilation of available experimental reports.

\section{E. Theoretical tools}

\section{Structural calculations for heteronanotubes}

Because of the large number of atoms and long time scales involved in an adequate description of the properties of nanotubes, calculations can be very time consuming, expensive, and difficult to perform. The methods commonly adopted for large-scale calculations of the electronic properties of heteronanotubes of $\mathrm{B}, \mathrm{C}$, and $\mathrm{N}$ (as in the case of other molecular nanostructures) were mostly adapted from the theories available for materials at the macroscopic and mesoscopic scales. Such computational schemes are based on the solution of the many-body Schrödinger equation. While the problem is completely defined in terms of the total number of particles $N$ and the external potential $V(r)$, its solution depends on $3 N$ coordinates. This makes a direct search for either exact or approximate solutions to the many-body problem a task of rapidly increasing complexity. The alternative density-functional theory (DFT) formulation, based on the particle density rather than on the manybody wave function, is feasible and has been rather successful. Within DFT, the ground-state energy of an interacting system of electrons in an external potential can be written as a functional of the ground-state electronic density (Kohn, 1983, 1999; Dreizler and Gross, 1990; Fiolhais et al., 2003). When compared to standard quantum chemistry methods, this approach is particularly appealing, because it does not rely on complete knowledge of the $N$-electron wave function, but only on the electronic density. However, although the theory is exact, the energy functional contains an unknown quantity called the exchange-correlation energy that must be approximated in practical implementations. Although failures of DFT are known, its use continues to increase because of its the better scaling with the number of atoms and the fact that failures are connected with a particular choice of the local functional, with the possibility of improving the accuracy as more and more sophisticated exchange-correlation functionals are generated. 


\section{Theoretical methods for spectroscopic tools}

There are several spectroscopic techniques that are essential tools in characterization of complex systems at the atomic scale. Structural properties are routinely studied using techniques such as XRD and STM. From a theoretical point of view, the standard first-principles method is DFT using the local (LDA) and semilocal generalized gradient (GGA) approximations to the exchange-correlation effects (XC) (Kohn, 1983, 1999; Fiolhais et al., 2003). DFT allows efficient calculation of the ground-state properties of any electron system and consequently of its optimal structure. The popularity of DFT is, undoubtedly, one of the major achievements of theoretical condensed matter physics. Its use has been extended to other fields like biology, and in particular to the study of the structural and electronic properties of nanotubes relevant to the present review. The basic tools in theoretical $a b$ initio spectroscopy are DFT, both static DFT and dynamic time-dependent (TDDFT), and many-body perturbation theory (MBPT). Static DFT has reached an impressive level of reliability and efficiency [see Onida et al. (2002) as well as Marques et al. (2006), and references therein].

The existing approximations to the electron exchange and correlation terms are not satisfactory in predictions of structural properties of complex systems (for instance, the description of van der Waals forces, reaction barriers, or highly correlated systems). TDDFT is also affected by this general limitation, with the added difficulty of requiring the correct description of the dynamic effects within the $\mathrm{XC}$ potential. The latter is indispensable for analysis of nonlinear phenomena (where the response of the electron system to an external probe, such as an intense x-ray beam, cannot be described by treating the beam as a perturbation, e.g., femtosecond or attosecond dynamics). In the linear response regime there has been interesting progress in the representation of the dynamic part of the XC kernel, which has provided a method to describe excitonic effects in different nanomaterials. This has been applied, for instance, to the optics of BN nanotubes, which is fully determined by excitonic effects (Park et al., 2006; Wirtz et al., 2006). MBPT avoids the density-functional description and offers a robust and in principle exact theoretical alternative for the treatment of structural and dynamical properties (quasiparticle and neutral excitations). The disadvantage is the higher computational cost of this approach. In this framework, the one-particle Green's function is usually evaluated at the $G W$ level, and the state of the art for the study of neutral excitations is based on the resolution of the Bethe-Salpeter equation.

\section{STRUCTURAL AND MORPHOLOGICAL ANALYSIS OF HETERONANOTUBES OF B, C, AND N}

\section{A. Overall morphology}

The synthesis methods and their success in producing different heteronanotubes were described in detail in Sec. III, and the most common characterization tools for understanding the sample structure in Sec. IV. These descriptions made it clear that the material available is still limited and the synthesis conditions still need to be improved. Although many have accomplished synthesis of nanotubes in an atmosphere containing atoms of the desired dopant agents, this does not imply that the products are doped nanotubes. The morphology, composition, and elemental distribution of these nanotubes are controlled by a number of parameters, namely, the operating conditions, precursors, and catalytic system. Radical differences in bright-field TEM imaging for these kinds of nanotubes have not been conclusively reported. Raman spectroscopy seems to be sensitive to the introduction of dopants, but in this case the reference sample needs to be properly selected (Rao et al., 1997). In this context Golberg, Bando, Burgeois, et al. (2000) performed high-resolution imaging and ED on B- and N-doped C-SWNTs bundles in order to perform a population analysis of the helicities in such samples. Based on the theoretical predictions, increases in the zigzag nanotube population for B-doped nanotubes were expected; however, no preferential helicity could be determined for any of these samples. Arenal, Kociak, et al. (2006) analyzed 121 boron nitride single-walled nanotubes with TEM studies, finding that $12 \%$ of the tubes had zigzag chirality, while the distribution of the other helicities was uniform. A typical diffraction pattern is shown in Fig. 19. In that contribution, they also studied BN-DWNTs and observed that the value of the intertube distance and the significant difference of the chiral angle between the two tubes indicated a lower interaction between the $\mathrm{BN}$ sheets than in bulk hexagonal BN. In general, samples imaged in bright field show the high quality of the samples but do not exhibit many differences. As a proof of these one can see the typical examples of Band $\mathrm{N}$-doped nanotubes in Figs. 11, 13, and 23. Images from BNC-SWNTs and BN-SWNTs can also be found in Figs. 10 and 12. The factor limiting the ability to distinguish the different atoms in nanotubes containing $\mathrm{B}, \mathrm{N}$, and/or $\mathrm{C}$ is this close $Z$ values. However, this kind of direct observation will most likely be possible in the not so distant future.

Once the single-walled morphology of the nanotube has been identified, which is nowadays a routine task, the identification of the dopant distribution and localization is the next task. Imaging combined with a highly spatially resolved spectroscopy would be the ideal situation.

\section{B. Determination of doping amounts and dopant distribution}

Almost five decades ago analysis of solid carbon systems broke new ground by analysis of the incorporation of nitrogen within a pyrolyzed bulk carbon structure (Zanchetta and Marchand, 1965). A subsequent thermal annealing within the same structure revealed the feasibility of reaching low dopant concentrations $(<0.5$ at. \%). With these kinds of bulk systems, the finding of dopants is no longer technologically limited. However, in molecular nanostructures such as those we study 
here, the determination of doping amounts and dopant distribution is not a straightforward task and still relies on the development of technological tools.

This section discusses the distribution of $\mathrm{C}, \mathrm{B}$, and $\mathrm{N}$ atoms throughout the structure of a SWNT. In the particular case of doped C-SWNTs with low dopant concentration, most studies have used an indirect method to indicate the amount of heteroatom incorporation, and one must pay close attention to this peculiarity. For instance, it is well known that $\mathrm{N}_{2}$ and other gaseous synthesis by-products can get trapped inside the hollow core of N-doped nanotubes (Reyes-Reyes et al., 2004; Ayala, Grüneis, Kramberger, et al., 2007). If the tools employed and the interpretation of results are not adequate, $\mathrm{N}$ in different gaseous forms could be mistakenly considered as a wall dopant. For the determination of the dopant amount and distribution, TEM-EELS, EELS, XAS, and XPS are the key techniques since they enable direct determination of the relative and absolute doping amount in combination with a sensitivity in the percentage range. This, however, strongly depends on the element studied, since one has to keep in mind the element-specific inelastic scattering cross section, as well as the photoionization cross section. In addition, these techniques allow an analysis of the dopant distribution and bonding environment.

For N-doped carbon nanostructures, Belz et al. (1998) reported the introduction of up to 4.5 at. \% of $\mathrm{N}$ within $s p^{2}$-like carbon environments. A recent study from Alibart et al. (2008) analyzed the semiconducting properties of these kinds of films upon $\mathrm{N}$ incorporation after synthesis with different techniques. Many subsequent studies on $\mathrm{N}$-doped bulk graphitic systems have been made and have shown that $\mathrm{N}$ atoms tend to introduce disorder in the graphene planes. Recent theoretical studies showed how nitrogen substitution within the carbon lattice tends to introduce curvature effects on the graphitic sheets (Gammon et al., 2003). B-doped carbon systems have also been investigated, but at a more limited level. Shirasaki et al. (2000) showed that the limiting value of the boron concentration, for boron-substituted carbon nanostructures prepared by thermal CVD, decreases as the reaction temperature increases. They attributed this to the possibility that boron-substituted carbon structures with high boron content might be metastable phases.

Of course, bulk carbon structures drastically differ from nitrogen- and boron-doped carbon-based nanostructures such as graphite (Shimoyama et al., 2000), graphene (Lherbier et al., 2008), nanoribbons (Yu et al., 2008), fullerenes (Yang, 2008), and nanotubes, but these are only a few examples of the vast research that has been carried out with carbon systems and the incorporation of boron and nitrogen into them.

The dopant distribution is related to the localization of the dopant agents, or nanodomains throughout the tubular structures. The most straightforward methods to accomplish this are spatially resolved analytic techniques. Typical examples for EELS dopant detection are shown in Figs. 10, 11, 14, 16, 20, and 21. The doping
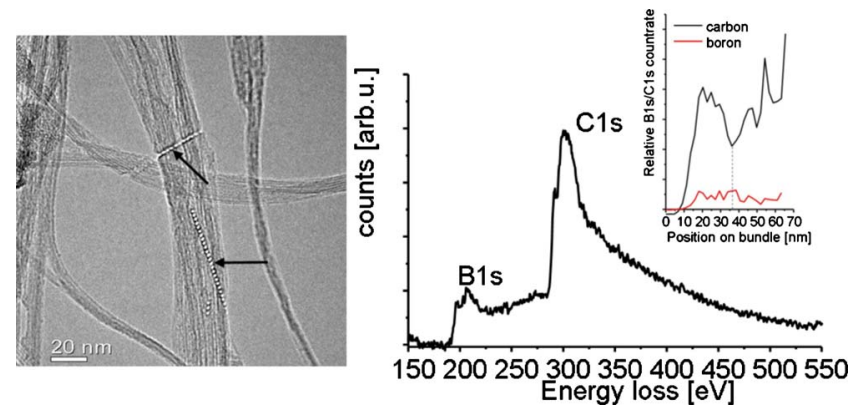

FIG. 20. (Color online) Spectrum line recorded, after striapping the background, across a bundle of B-doped SWNTs. The inset shows the B:C ratio. Adapted from Borowiak-Palen et al., 2003.

amount can be easily elucidated as shown, for instance, in Figs. 20 and 21 for EELS and Fig. 22 and 23 for XPS. In recent years this identification has been especially successful with BNC-SWNTs and BNC-MWNTs (Enouz et al., 2006, 2007; Enouz-Védrenne et al., 2008). Fewer studies can be found for B- and N-doped C-SWNTs. Krstic et al. (2007) and Lin et al. (2008) perfomed a detailed study of the local distribution of $\mathrm{N}$ atoms in $\mathrm{N}$-doped SWNTs grown by arc discharge and laser vaporization, respectively. At the maximum $\mathrm{N}$ content of $1 \%$ they found a random distribution of $\mathrm{N}$ donors in the SWNT. An example of N-doped C-SWNTs grown via laser vaporization is shown in Fig. 14. Gai et al. (2004)
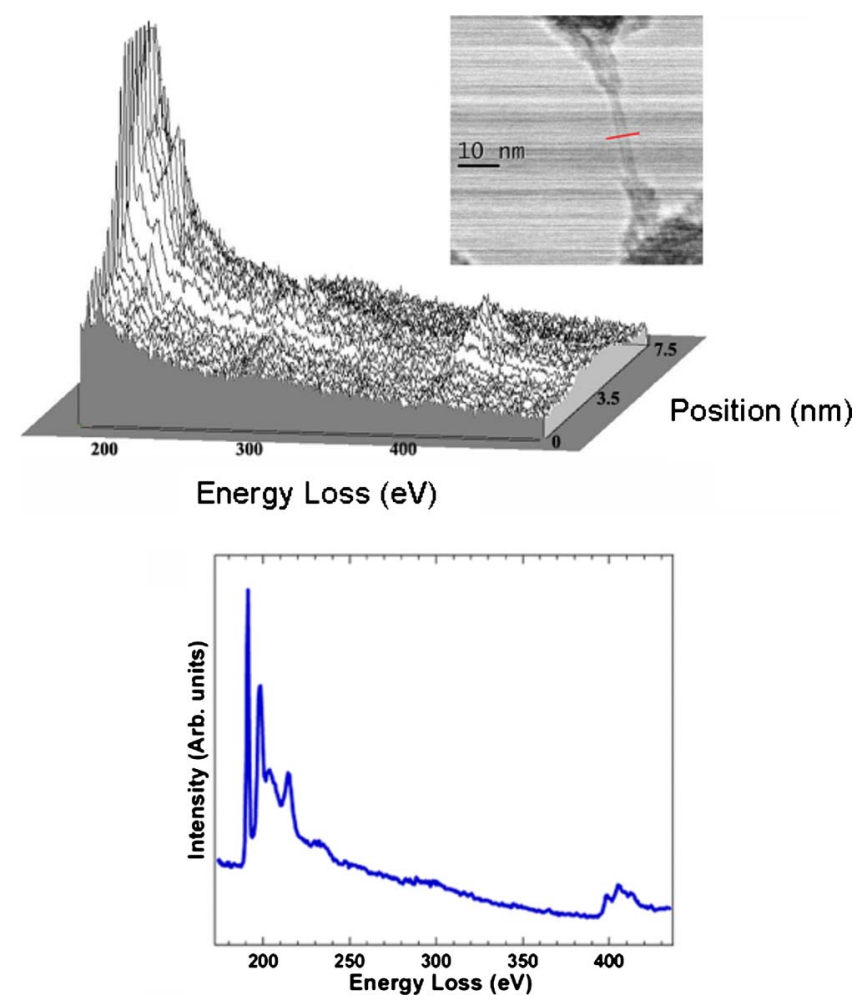

FIG. 21. (Color online) Spectrum recorded across a BNSWNT shown in the bright-field image above. The lower panel depicts the fine structures in the B and $\mathrm{N}$ edges. Adapted from Arenal et al., 2005. 

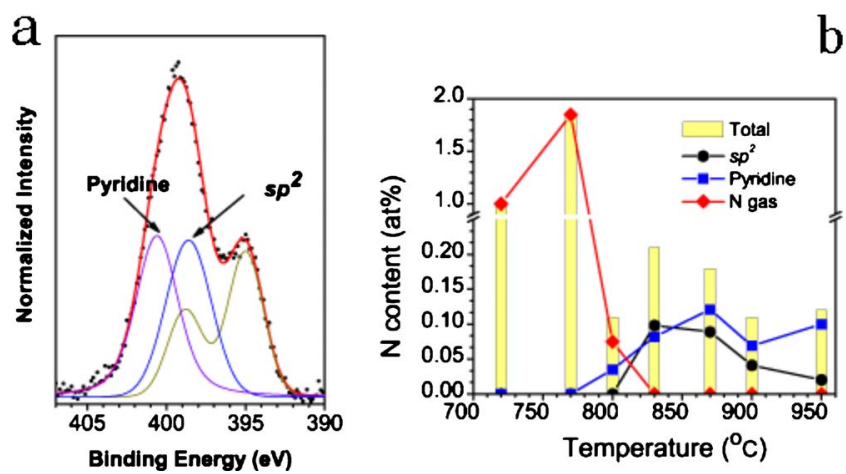

FIG. 22. (Color online) XPS is a ley method to analyze core level excitations. This method can be used to detect heteroatom percentages as low as $0.2 \%$ at., which is the case corresponding to these figures. (a) The $\mathrm{N} 1 s$ core signal corresponding to $\mathrm{N}$-doped C-SWNTs synthesized from benzylamine chemical vapor deposition with a line-shape analysis that shows the presence of pyridinic and $s p^{2}$-like bonding environments in equal proportion at certain synthesis conditions. (b) Nitrogen incorporation profile according to synthesis temperatures. The gray bars correspond to the total $\mathrm{N}$ content at different synthesis temperatures. The fractions from the lineshape analysis $\left(s p^{2}\right.$, pyridine, and $\mathrm{N}$ volatile species) are depicted by the symbols specified on the top right labels. Adapted from Ayala, Grüneis, Gemming, Grimm, et al., 2007.

used SR-EELS to study B-containing C-SWNTs samples synthesized by laser ablation, where they could not directly identify the presence of $B$ within nanotube bundles produced from targets containing from 1.5 to $10 \%$ of B. They assumed a very low doping, below the instrument detection limits, and attributed the absence of B in the bundles of C-SWNTs to preferential incorpo-
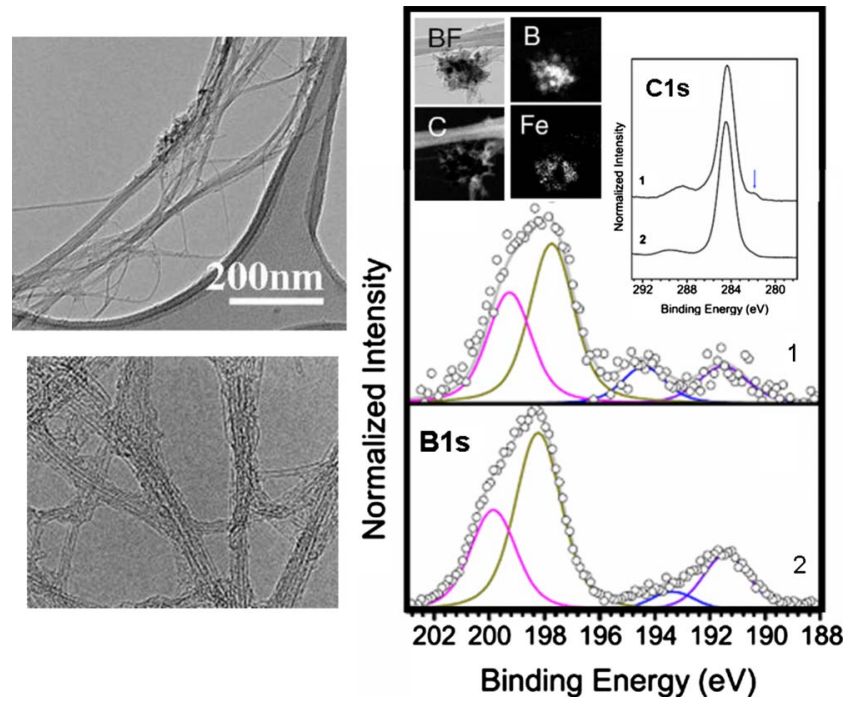

FIG. 23. (Color online) B-doped SWNTs have been successfully synthesized with CVD. The left side images show low and high magnification TEM micrographs of such kind of material. On the right panel, C $1 s$ and B $1 s$ XPS signal of the B-SWNT at two different temperatures (1) $890{ }^{\circ} \mathrm{C}$ and (2) $840{ }^{\circ} \mathrm{C}$. The inset shows the EFTEM for $\mathrm{C}, \mathrm{B}$, and Fe together with the BF image. Adapted from Ayala et al., 2008. ration in nanoparticles of $\mathrm{B}_{4} \mathrm{C}$, which were detected in a sample synthesized from a target containing $10 \%$ of $\mathrm{B}$.

The B-doped C-SWNT bundles produced by Borowiak-Palen et al. (2004) via the substitution reaction are other typical examples. A random example is shown in Fig. 20 for an average B content of 15\%. TEM characterization after the substitution reaction revealed the presence of high-purity nanotube bundles with a homogeneous diameter distribution. They emphasized the use of substitution reactions to achieve higher B dopant incorporation. They performed a TEM-EELS analysis with $10 \mathrm{~nm}$ beam size, which showed that a localized substitution level could reach values up to 25 at. \%, and that the $\mathrm{B}$ content shows strong scattering at this high heteroatom concentration. It will be clarified later in more detail how high doping levels imply a strong change of the C-SWNT band structure and consequently the formation of new heteronanotubes with new properties. Therefore these "highly doped nanotubes" have to be considered as heteronanotubes and not as B-doped C-SWNTs.

Another demonstrative example is provided by $\mathrm{BN}$ SWNTs. The use of a dedicated STEM, allowed to develop SR-EELS studies, was crucial to establish that $\mathrm{BN}-\mathrm{NTs}$, synthesized by the laser vaporization technique, corresponded to an $s p^{2}$-bonded $\mathrm{BN}$ structure where boron and nitrogen were uniformly distributed in the tube wall in a 1:1 ratio. These results are shown in Fig. 21. This figure shows a spectrum line (SPLI) recorded across a BN-SWNT (bright-field image in the inset) and an EELS spectrum extracted from this SPLI where the fine structures of the boron and the nitrogen $K$ edges are visible (lower panel).

\section{Bonding environments}

The properties of carbon-based thin films have been analyzed on relation to variations of the $s p^{2}$-to-s $s p^{3}$ carbon bonding ratios. For instance, Franceschini et al. (1992) confirmed internal stress reduction by nitrogen incorporation in hard amorphous carbon thin films. Further studies with XPS and Rutherford backscattering spectroscopy (RBS) have also been extensively performed in such films (Hellgren et al., 1999, 2001; Gammon et al., 2003). Studies in low-nitrogen-doped highly oriented diamond films have suggested that $\mathrm{N}$ doping stops vacancies from aggregating, inducing the formation of vacancy clusters. For Jacobsohn et al. (2004) performed an extensive XPS study on boron carbide films deposited by sputtering.

Nanotubes are relatively new materials, and because few results are available for comparison with doped nanotubes in this framework, many of the results above have been used for comparison with $\mathrm{N}$-doped nanotube samples. In particular, the identification of bonding environments for $\mathrm{N}$-doped tubes has been based on results on bulk doped systems. Because of the differences that nanotubes must present due to their molecular nanostructure nature, special attention should be given to those kinds of analysis. C-NTs and in particular 
C-SWNTs are extremely sensitive to localized nitrogen incorporation, and this is the reason that completely new possibilities, but also difficulties, enter this scenario. Not only the amount of $\mathrm{N}$ incorporated in the nanotubes, but also the bonding environments of the foreign atoms must be analyzed.

Experimental research regarding $\mathrm{N}$ - and $\mathrm{B}$-doped C-SWNTs is still in its initial stage because of the availability of samples. Bulk probing methods (XPS with different photon energies) have been used in $\mathrm{N}$-doped C-DWNTs and mixtures of SW and DW material (Kim et al., 2005; Ayala, Grüneis, Gemming, Büchner, et al., 2007; Ayala, Grüneis, Gemming, Grimm, et al., 2007; Ayala, Grüneis, Kramberger, et al., 2007; Panchakarla et al., 2007; Elias et al., 2010). The sensitivity (resolution) to the different bonding environments has allowed identification of the different ways in which $\mathrm{N}$ incorporates according to the synthesis conditions. Ayala, Grüneis, Gemming, Grimm, et al. (2007) and Elias et al. (2010) determined a maximum incorporation of 0.2 and 0.3 at. \% of $\mathrm{N}$ in samples produced with benzylamine $\left(\mathrm{C}_{7} \mathrm{H}_{9} \mathrm{~N}\right)$. Although these experiments were done in completely different systems, and the overall morphology of the samples is different, the incorporation limits and the bonding environment configuration exhibit a good correlation. The chart on the right side of Fig. 22 is a nitrogen incorporation profile based on the deconvolution of $\mathrm{N}$ incorporation spectra registered for samples produced at different synthesis conditions (left panel of Fig. 22). Shown there is how, in the absence of $\mathrm{N}$ gaseous forms, the direct substitution and pyridinic forms compete. In a completely different system, and using $\mathrm{CH}_{4}-\mathrm{NH}_{3}$-Ar mixtures, Kim et al. (2005) identified a 1:1 ratio in the graphitelike and pyridinelike $\mathrm{N}$ structures. They also performed a self-consistent charge-densityfunctional-based tight-binding calculation, showing that if the $\mathrm{N}$ doping occurs on the inner wall a more stable DWNT is formed than if the outer wall contains the dopants. There are no doping amount reports at the local scale with C-SWNT samples doped either with $\mathrm{N}$ or with $\mathrm{B}$ at dopant concentrations as low as the ones reported for C-SWNT by Ayala, Grüneis, Gemming, Grimm, et al. (2007) and Elias et al. (2010).

Recently $\mathrm{CN}_{x}$-SWNTs synthesized via the laser vaporization technique have been successfully studied with local probing techniques. Lin et al. $(2008,2009)$ studied the $\mathrm{N}$ local environment in individual as well as in bundles of $\mathrm{CN}_{x}$-SWNTs via the analysis of the fine structures near absorption edges. They found the $\mathrm{N}$ local arrangement for a mean nitrogen content of 1.7 at. \% to be a mixture of pyridinelike and graphitelike configurations. Similar results were obtained by Glerup et al. (2004) working on $\mathrm{CN}_{x}$-SWNTs produced via arc discharge.

The case of boron has been investigated theoretically extensively in comparison to nitrogen. However, the experimental results have been in the inverse proportion. The difficulties in synthesis of B-doped nanotubes are mainly related to the rich boron chemistry related to the most common catalysts. If $\mathrm{B}$ is regarded as a dopant agent in a C-SWNT, there are two possibilities. The first one is to substitute directly for the carbon atoms in the lattice. The second is to form nanodomains or "islands" of boron atoms; these have not been identified experimentally. Only an $s p^{2}$ configuration for the substitutional boron atoms is expected. This eases the spectroscopic studies, which usually require a deconvolution of the core signal to discriminate between bonding environments. In CVD (Ayala et al., 2008) as well as laser ablation (Gai et al., 2004) production of B-doped C-SWNTs, it has been observed that $\mathrm{B}_{4} \mathrm{C}$ is likely to form; this is practically the only competing carbonaceous structure containing boron that exists as a co-product. Much less has been reported in this case than for N-doped SWNTs. Therefore, a detailed insight into the photoemission response corresponding to the $\mathrm{C} 1 \mathrm{~s}$ level is extremely important in the characterization of $\mathrm{B}$ doping and $\mathrm{B}$ content. In particular, for $\mathrm{B}_{4} \mathrm{C}$ systems, this feature becomes more prominent and shifted so that the $\mathrm{C} 1 \mathrm{~s}$ spectrum presents two well-defined peaks separated by $\sim 3 \mathrm{eV}$. In this respect it is worth mentioning that, as in $\mathrm{N}$ doping, the majority of the work reporting the synthesis of low B doping in single-walled nanotubes had estimated the possible B amounts solely based on the B content of the material employed for synthesis (i.e., $\mathrm{C}$ targets containing $\mathrm{B}$ compounds in the preparation mixture) until recently reported XPS studies (Ayala, Reppert, et al., 2010). This yields a strong overestimation of the actual $s p^{2}$-bonded $\mathrm{B}$ content. To understand this better it is useful to look at Fig. 23: the B $1 s$ and $\mathrm{O} 1 s$ responses for two different samples synthesized with the same catalyst treatment and precursor conditions but at different temperatures $\left(890\right.$ and $\left.840{ }^{\circ} \mathrm{C}\right)$. First, the $\mathrm{O} 1 s$ signal confirmed the formation of a carbonaceous nanotube species. No upshift or downshift of this main peak in these $\mathrm{CB}_{y}$ systems has been observed. However, in the first case a shoulder at $281.8 \mathrm{eV}$ was observed. Shirasaki et al. (2000) showed that first-neighboring boron atoms could be responsible for this feature. This suggests that low substitutional doping with $\mathrm{B}$ atoms within the graphitic lattice of C-NTs (rather than heteronanotube formation with high amounts of $\mathrm{B}_{4} \mathrm{C}$ ) occurs at lower synthesis temperatures in the method proposed. For the B $1 s$ signal shown in the same figure, the major feature observed at higher binding energies is an asymmetric peak that can be associated with at least two distinct chemical states always present at around 198 and $199.5 \mathrm{eV}$. These cannot be associated with B-C chemical bonds and no such peaks have been reported, although a link to the formation of a compound containing $\mathrm{B}$ and $\mathrm{Fe}$ cannot be ruled out. Analytical TEM is a useful tool in this case [see the EFTEM results in the inset in the right panel of Fig. 23 (Ayala et al., 2008)]. They performed elemental mapping in these low-nanotube-yield samples to focus specifically on the nature of the catalyst remaining, and concluded from these combined EFTEM and XPS studies that additional by-products were formed, such as a high formation of layers of $\mathrm{B}$, poisoning the catalysts, and inhibiting the growth of NT material. The maximum doping level in the substitutional $s p^{2}$ configuration ob- 


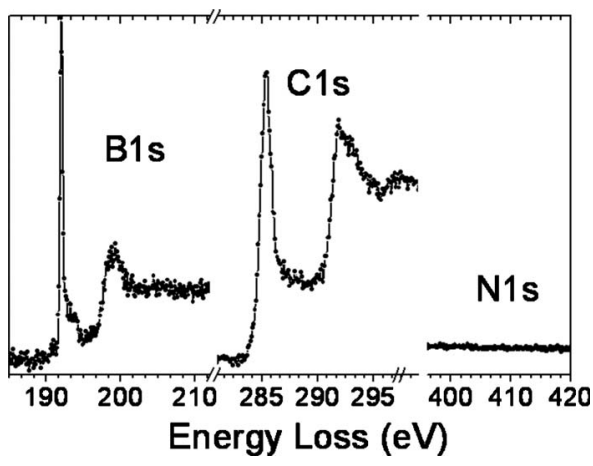

FIG. 24. Spectra corresponding to high-resolution EELS core level excitation spectra of B-containing C-SWNT. This material corresponds to B-doped SWNT in the range of the $\mathrm{B} 1 s, \mathrm{C}$ $1 s$, and N $1 s$ edges. Adapted from Borowiak-Palen et al., 2004.

served was about $1 \%$. Furthermore, it is highly unlikely that B-containing gaseous species form. Recently Panchakarla et al. (2007) reported the presence of the B 1 s signal at $191.4 \mathrm{eV}$ in C-DWNTs. In this case, the presence of an asymmetric peak suggests at least three chemical states that have not been identified. However, they suggested that for C-NTs the cylindical configuration and electron quantum confinement predict the B substitutional configuration at higher binding energy values than those for pure $\mathrm{B}(\sim 187 \mathrm{eV})$ and $\mathrm{B}$ in doped carbons $(\sim 190 \mathrm{eV})$. They also suggested that the signals at 191.5 and $192.1 \mathrm{eV}$ should be attributed to the substitutional configuration of B within the C-NTs. In summary, these results highlight that only B-doped samples at small doping can be realized via CVD and laser ablation and for excess $\mathrm{B}$ in the feedstock the amount of by-products, but not the actual yield of B-doped SWNTs, is increased.

The story completely changes for B-doped SWNTs grown by the substitution reaction. For nanotubes grown by this method, after purification (as shown in Fig. 24) no by-products, and especially no $\mathrm{N}$ contamination or formation of $\mathrm{BCN}$ nanotubes, are observed. The B $1 s$ spectrum of B-doped C-SWNT shows an intense peak at $192 \mathrm{eV}$ assigned to the $1 s \rightarrow \pi^{*}$ resonance and a less intense double peak at $199 \mathrm{eV}$ due to the $1 s \rightarrow \sigma^{*}$ resonance. The small shoulder at $194 \mathrm{eV}$ can be assigned to residual $\mathrm{B}_{2} \mathrm{O}_{3}$ from the precursor material. The $\mathrm{C} 1 \mathrm{~s}$ spectrum of B-doped C-SWNTs shows the excitonic $1 s$ $\rightarrow \pi^{*}$ absorption at $285.3 \mathrm{eV}$, as in the case of the C-SWNT reference, and the corresponding $1 s \rightarrow \sigma^{*}$ absorption at $292 \mathrm{eV}$. The spectral shape of the $\mathrm{C} 1 s \rightarrow \pi^{*}$ and the $\mathrm{C} 1 s \rightarrow \pi^{*}$ resonances of the B-doped C-SWNT resemble quite closely those of the pure material, indicating that the boron substitution does not alter the chemical environment of the carbon atoms of the tube significantly. This highlights again that $s p^{2}$-bonded B can be incorporated up to an average doping level of at least $15 \%$.

Similar information can be extracted from spatially resolved TEM-EELS as shown in Fig. 20 for B-doped SWNT and Fig. 21 for BN-SWNTs. For instance, for
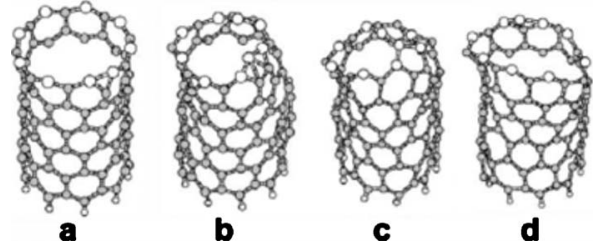

FIG. 25. Snapshots of ab initio molecular dynamics (MD) calculations revealing the formation of B-doped SWNTs with zigzag chirality $(9,0)$. B acts as a surfactant and inhibits tube closure, thus enhancing the length of the tubules. From Blase et al., 1999.

BN-SWNTs the different B edge is clearly distinguished and again allows an unambiguous assignment of the $s p^{2}$ hybridization of the incorporated $\mathrm{B}$ and $\mathrm{N}$ atoms. Because graphene and $h$ - $\mathrm{BN}$ are anisotropic materials, the fine structure of the $\mathrm{B}, \mathrm{C}$, and $\mathrm{N} K$ edges strongly depends on the orientation of momentum transfer $(q)$ with respect to the lattice (Leapman et al., 1983; Keast et al., 2001; Arenal, Kociak, and Zaluzec, 2007). Thus the chemical bonding maps (as shown, for instance, in Fig. 21) of the $s p^{2} \mathrm{BN}$ lattice in the two extreme orientations (the electron beam parallel and perpendicular to the anisotropic axis) provide additional proof that BN-NTs consist of an $s p^{2}$-bonded structure distribution at a nanometer scale. Furthermore, the nature of the chemical environment and chemical bonding can be extracted at least qualitatively from the fine structures near the absorption edges. For B-C-N nanotubes, inspection of the $\mathrm{C}, \mathrm{N}$, and $\mathrm{B} K$ edges and their fine structures is required. Spatially resolved (SR) spectroscopic information can be recorded using a scanning TEM (STEM) under a particular acquisition mode called spectrum imaging (SPIM) or spectrum line (SPLI). These modes consist in the acquisition of one EEL spectrum for each position of the probe scanning over a 2D region (SPIM) or a line (SPLI) at the surface of the nanotubes and nanoparticles. Enouz et al. (2007) investigated BCN-SWNTS using this local probe technique. These NTs are a new kind of SWNT made of graphene and BN pieces selforganized in a sequential manner along the tube axis.

\section{TENTATIVE GROWTH MECHANISMS}

Although several growth mechanisms for C-NTs have been proposed, there is no universal growth method generally accepted so far, and the ideas proposed are much more scarce for heteronanotubes. Nevertheless, we can cite some of the proposed studies for the structures we analyze here.

Figure 25 represents molecular dynamics calculations that suggest that B-doped C-SWNTs mainly follow zigzag chirality during growth. Blase et al. (1999) showed in this study the construction of a pentagon formed by three $\mathrm{B}$ atoms and two $\mathrm{C}$ atoms, as well as pure $\mathrm{C}$ pentagons. The first case leads to partial tube closure. For pure $\mathrm{C}$, the tube would close almost immediately, but the presence of $\mathrm{B}$ prevents this and the $\mathrm{B}-\mathrm{B}$ bond breaks 
and rearranges along the opposite edge of the tube. They suggested that B atoms prevent closure of the tubes in the zigzag case. The partical tip closing of B-doped C-MWNTs has been reported experimentally, confirming these theoretical predictions. Thus the predictions of Blase et al. (1999) were confirmed experimentally and have paved the way toward more extensive semiempirical simulations of boron-assisted nanotube growth (Hernández et al., 2000; Terrones, Terrones, et al., 2000; Wiltshire et al., 2005).

Not much has been proposed for $\mathrm{CN}_{x}$-SWNTs. Sumpter et al. (2009) recently proposed a three-step growth mechanism for the CVD synthesis of N-doped C-SWNTs. The first corresponds to a still unclear nucleation of the SWNT, followed by the incorporation of N atoms through the open edges, and a later inward bending of the nanotube wall produced by a higher concentration of $\mathrm{N}$ at the open edge. According to these authors, the last step leads to either a subsequent closure of the nanotube layer due to higher concentration of $\mathrm{N}$ or a frustration in the closure of the tube and subsequent corrugation as the nanotube keeps growing. For $\mathrm{CN}_{x}$-MWNTs it is believed that with an increase of $\mathrm{N}$ the number of walls within decreases and the proportion of pyridinelike $\mathrm{N}$ increases (Terrones et al., 1999). The pyridinelike $\mathrm{N}$ sites are suggested to be responsible for the roughness and the interlinked morphologies. Srivastava et al. (2004) performed tight-binding calculations and suggested that $\mathrm{N}$ incorporation should be possible for nanotube with diameters larger than $8 \mathrm{~nm}$. They suggested that vacancy-mediated $\mathrm{N}$ substitution is favored on the surface of such large-diameter nanotubes. Kang and Jeong (2004) performed theoretical calculations using DFT on nanotubes with different chiralities. They also proposed that $\mathrm{N}$ atoms in armchair NTs agglomerate on the equatorial axis, promoting NT instability. It is clear that theoretical studies on $\mathrm{CN}_{x}-\mathrm{SWNTs}$ require additional calculations, as the above-mentioned predictions do not support the recent experimental results.

Recently Arenal, Stephan, et al. (2007) proposed a root-growth model for BN-NTs, which involves the presence of a boron droplet. This phenomenological model considers the solubility, solidification, and segregation phenomena of the elements present in this boron droplet. In this model, they distinguished three different steps as a function of temperature: formation of the liquid boron droplet from the decomposition of different boron compounds existing in the hexagonal boron nitride target, reaction of these boron droplets with nitrogen gas present in the vaporization chamber and recombination of these elements to form boron nitride, and incorporation of the nitrogen atoms at the root of the boron particle at active reacting sites, which achieves the growth of the tube.

The study by Arenal and co-workers suggested that oxygen "poisons" the BN-SWNT growth. In this context Blase et al. (1998) used first-principles molecular dynamics to consider the stability of B-N bonds as compared to $\mathrm{B}-\mathrm{B}$ or N-N bonds. They showed that armchair BNSWNTs can develop an ordered metastable edge, whereas the zigzag ones rapidly evolve into an amorphous tip which prevents further growth. Those predictions were confirmed experimentally (Wiltshire et al., 2005) and have also paved the way toward more extensive semiempirical simulations of boron-assisted nanotube growth.

A phenomenological model for the growth of BNCSWNTs has also been proposed by Enouz et al. (2007), who considered that the formation of these kinds of tubes was owing to the segregation phase existing at equilibrium between graphite and $h$-BN.

As mentioned, not even the growth models of C-SWNTs are fully understood and great controversy still exists. Many more models are expected in the case of heteronanotubes of $\mathrm{B}, \mathrm{C}$, and $\mathrm{N}$.

\section{ELECTRONIC STRUCTURE AND TRANSPORT}

In this section, we review the available studies of the electronic transport properties. As mentioned, we have to distinguish between two cases: doped C-SWNTs and heteronanotubes. The key to separating these two systems is a detailed analysis of the modification in the bonding environment and the corresponding doping amounts yielding different charge distributions.

In this section it is also important to keep in mind that the literature regarding transport in $\mathrm{B}, \mathrm{N}$, and $\mathrm{C}$ heteronanotubes is mainly restricted to theoretical calculations. Some experimental results have been published for multiwalled and for $\mathrm{N}$-doped structures, but singlewalled material has only recently become available (Krstić et al., 2007; Murata et al., 2008).

\section{A. N and B substitutionally doped C-SWNTs}

Lammert et al. (2001) showed that under doping with boron and nitrogen the microscopic doping inhomogeneity of C-NTs is larger than in normal semiconductors leading to charge fluctuations that can be used to design nanoscale devices (diode behavior). In such structures, new acceptor and donor features are observed in the local density of states (LDOS) of the valence and conduction bands when compared to graphene. The role of the $\mathrm{B}$ or $\mathrm{N}$ atoms in the electronic structure of modified $s p^{2}$-hybridized carbon networks has been treated using different theoretical approaches.

If $\mathrm{N}$ substitutes directly for $\mathrm{C}$, an $n$-type semiconductor with sharp localized states above the Fermi level is generated. Because of its size, $\mathrm{N}$ can also generate a defect in the tube structure, keeping the heteroatoms on the walls but requiring a rearrangement of the neighboring $\mathrm{C}$ atoms. In this case the $n$-type behavior cannot be inferred immediately as in the case of direct $\mathrm{N}$ substitution [see Fig. 5(a)]. One cannot discard a second configuration which could involve different wall rearrangements. The electronic behavior depends on the new geometry generated and this new wall structure could be such that $p$-type doping is also feasible. Czerw et al. (2001) suggested that this second possible bonding 
environment of nitrogen should correspond to a pyridinic configuration [see Fig. 5(b)]. They used tightbinding and $a b$ initio theoretical calculations, as well as experimental XPS and STM performed on bamboolike $\mathrm{CN}_{x}$ multiwalled NTs. This configuration suggests a twocoordinated $\mathrm{N}$ atom and creation of a single $\mathrm{C}$ atom vacancy responsible for the metallic behavior in those MW N-containing nanotubes. Further theoretical studies on doped single-walled CNT structures have also been carried out but no conclusive experimental proof of this configuration has been identified at the local scale. Kang and Jeong (2004) performed DFT calculations that indicated changes in the distribution of the nitrogen atoms according to different nanotube chiralities. They substantiate that the pyridinic configuration proposed by Czerw et al. (2001) is energetically favorable; however, they attribute it to a lone-pair state. Transport studies on single vacancies in CNTs by Rocha et al. (2008) support the previous study by calculating the DOS of impurities in CNTs upon progressive removal of $\mathrm{C}$ atoms in the structure. Although the substitutional configuration leads to an $n$-type material, theoretical tight-binding studies have suggested that this type of configuration can also give origin to a $p$ - or $n$-type structure depending on the atomic position of the $\mathrm{N}$ atoms. Nevertheless, none of these studies has shown compelling evidence of the creation of a vacancy induced by $\mathrm{N}$ incorporation nor of its configuration. Although bulk analysis of N-doped CNT samples with spectroscopic techniques has shown the presence of a pyridinic configuration. Further theoretical studies and experimental confirmation at the local scale are still expected within this framework.

Latil et al. (2004) published pioneering studies on how $\mathrm{B}$ and $\mathrm{N}$, observed as chemical impurities, affect transport regimes and length scales and revealed unexpected transport trends. These studies also explained the experimental reports on B-doped MWNTs by Krstic et al. (2003) on the effect of disorder on transport in these structures. Recent studies of the charge transport properties of individual $\mathrm{CN}_{x}$-SWNTs suggest that $n$-type conduction can be achieved but only when there is a high concentration of graphitelike nitrogen bonding (Krstic et al., 2007). They suggested that the pyridine-type nitrogen bonding configuration in the honeycomb lattice is the main contributor to the electric-dipole scattering process observed in charge transport. Furthermore, these studies confirmed the possibility that $\mathrm{CN}_{x}$-SWNTs could be $p$ - and $n$-type conductors as a function of the nitrogen bonding configuration (pyridine type or high concentration of graphitelike, respectively).

Looking at SWNTs with low doping of $\mathrm{B}$ and $\mathrm{N}$ as a class of weakly disordered low-dimensional systems, Latil et al. (2005) reported magnetotransport features under the application of a magnetic field parallel to the tube axis. They suggested that hole conduction is strongly affected by impurity scattering with short mean free path and negative magnetoresistance. They pointed out the difference from electron conduction with much

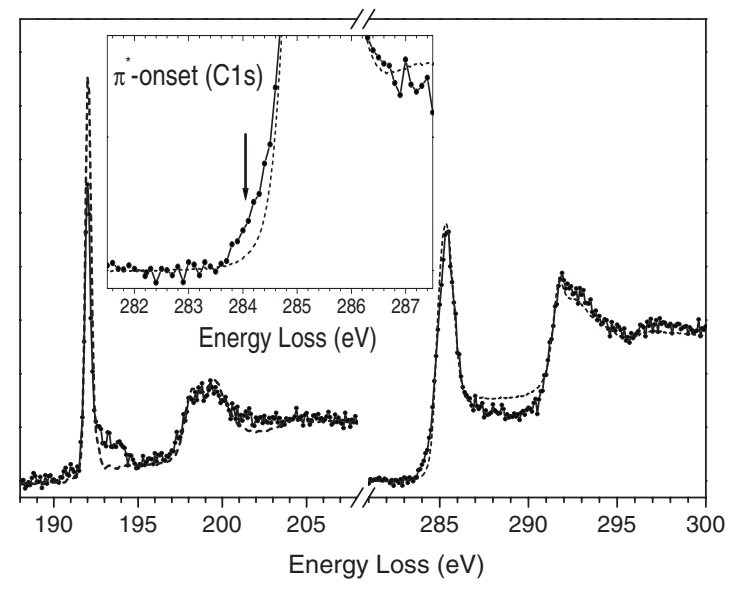

FIG. 26. Electronic properties of the unoccupied states highly B-doped SWNT determined by high-resolution core level EELS of the B $1 s$ and $\mathrm{C} 1 s$ edges. The inset shows the change of the $\mathrm{C} 1 s$ onset in an expanded scale. From Fuentes et al., 2004.

longer mean free path and positive magnetoresistance behavior.

For $p$-type doping Mele and Ritsko (1981) used a tight-binding approach to calculate the core exciton spectrum of graphite, and showed that the changes induced by low B substitution ( 0.5 at. \%) can be explained by a rigid-band-shift model, i.e., by assuming that the Fermi level is shifted downward because of the missing $\pi$ electrons from the boron atoms while the valence band structure remains otherwise undisturbed. For a $(10,0)$ nanotube with 1.25 at. \% B substitution, Yi and Bernholc (1993) calculated ab initio the formation of a shallow acceptor state at $0.16 \mathrm{eV}$ above the Fermi level. As discussed by Wirtz and Rubio (2003), these systems can be described at low doping to a first approximation within the framework of a rigid band model. At higher doping amounts, which can be reached only by substitution reactions and not by CVD or arc discharge, the local arrangement of the B dopants starts to play a crucial role in the stability and electronic structure of B-doped SWNTs. To date, all experiments on the electronic structure of B-doped SWNTs rely on direct local probes like TEM EELS (Borowiak-Palen et al., 2004) or on bulk probes like Raman spectroscopy (McGuire et al., 2005; Maciel et al., 2008) and EELS (Borowiak-Palen et al., 2003; Fuentes et al., 2004). The latter technique allows getting a detailed assignment of the bonding environment and charge distribution at the carbon and boron sites, respectively.

An example of a more detailed analysis of the B $1 s$ and $\mathrm{C} 1 s$ core excitation edges of the B-doped C-SWNT (solid) and the reference $\mathrm{B} 1 s$ and $\mathrm{C} 1 s$ spectra from a BN-MWNT (Fuentes et al., 2003) and a C-SWNT, respectively (dashed), is shown in Fig. 26. The inset in Fig. 26 represents an expanded scale focusing on the threshold of the $\pi^{*}$ resonance of the $\mathrm{C} 1 s$ edges of a B-doped C-SWNT (solid) and a pristine C-SWNT (dashed). The excitation spectra represent in a first approximation the 
projected matrix-element-weighted density of unoccupied states of each atom participating in the band structure. In addition, final-state effects of the remaining $1 \mathrm{~s}$ core hole lead to a strong redistribution of the spectral weight. For instance, it is well established that in the case of $1 s$ excitation edges of pristine C-SWNTs (Kramberger et al., 2007) and B-doped C-SWNTs (Fuentes et al., 2003) both the $\pi^{*}$ and $\sigma^{*}$ onsets are dominated by spectral weight resulting from the influence of the core hole. Thus, assuming a similar interaction between the excited electron and the core hole in B-SWCNT, one can expect that the $\pi^{*}$ resonances related to the DOS singularities of the different types of C-SWNT are washed out, resulting in the broad $\pi^{*}$ resonances. Nevertheless, the edges can be used to study some details in the electronic structure.

Comparison of the B $1 s$ spectrum of the B-doped C-SWNT with that of the BN nanotubes indicates that the chemical environment of boron is rather similar to that expected for an $s p^{2}$-hybridized environment, as it is also characteristic of $h$-BN, which constitutes experimental confirmation that the boron atoms are incorporated into the hexagonal lattice structure of the nanotube, in good agreement with theoretical predictions (Carroll et al., 1998; Lammert et al., 2001; Yi et al., 2006). The area below the $\pi^{*}$ resonance gives a measure of the charge transfer and bonding environment. The B $1 s \pi^{*}$ peak is about $13 \%$ lower in the B-doped C-SWNT than in the BN-MWNT, which shows that there is a different charge distribution within the B-C bonds that results in a higher electron density at the boron site. Turning back to the $\mathrm{C} 1 s$ excitation spectra, the inset of Fig. 26 provides evidence for the appearance of additional unoccupied states of carbon character in the electronic structure of the B-doped C-SWNTs, as shown by the lowenergy shoulder observed at the $\mathrm{C} 1 s$ excitation threshold and indicated by the arrow. The energy position of this feature can be roughly estimated as around $0.7 \mathrm{eV}$ below the corresponding $\mathrm{C} 1 s \rightarrow \pi^{*}$ threshold of the pure C-SWNTs. In analogy to $p$-type doping in graphite intercalation compounds (Mele and Ritsko, 1979), the presence of this doping-induced feature reflects the shift of the Fermi level of the B-doped C-SWNT to lower energies with respect to that of the C-SWNT. Although the corresponding total shift of the Fermi level from a comparison of both spectra alone can be complicated by additional effects like different excitonic effects or the mixture of semiconducting and metallic tubes, the observed shift by $0.7 \mathrm{eV}$ of the $\mathrm{C} 1 \mathrm{~s}$ excitation onset establishes a good estimate for the Fermi level shift, assuming a rigid band shift without strong changes of the C-SWNT band structure, as observed, for instance, for $\mathrm{FeCl}_{3}$-intercalated C-SWNT (Liu and Guo, 2004). Hence, the shift in the onset of spectral weight and the increase of the response of the $\pi^{*}$ resonance confirms that there exists a charge transfer from the boron atom to the C-derived bands. However, the analysis of Fig. 26 indicates that the shoulder is only about $6 \%$ of the total area, suggesting a charge transfer to carbon-derived states of $\sim 1 / 2$ hole per boron atom, which is lower than the estimation by Mele and Ritsko (1981) for B-doped graphite ( $\sim 0.8$ hole per B). This difference in the charge transfer also means a different charge delocalization around the B site and therefore is a hint for a different bonding environment in the B-doped C-SWNT than in B-doped graphite. In other words, the boron doping of C-SWNT cannot be solely explained within the framework of a simple rigid band model and additional changes in the nanotube band structure have to be taken into account. A typical example of the dependence of the band structure of B-doped SWNT on the amount of heteroatomic incorporation in the nanotube structure is shown in Fig. 27.

In summary, although there are some studies of B-doped SWNT at intermediate and high doping (5$20 \%$ ) the existence and stability of such structures is still heavily debated. It is likely that one has a B-doped C-SWNT only in the low-doping region and additional novel heteronanotubes in the case of high doping. This concept will be elucidated in detail in the next section.

\section{B. Heteronanotubes}

\section{Electronic structure of $\mathrm{BC}_{2} \mathrm{~N}$ and $\mathrm{BC}_{3}$}

Theoretically the band structure of $\mathrm{BC}_{2} \mathrm{~N}$ was first calculated by Miyamoto, Rubio, Cohen, and Louie (1994). Figure $28(\mathrm{~b})$ is a $(4,4) \mathrm{BC}_{2} \mathrm{~N}-\mathrm{SWNT}$ obtained by rolling the computed most stable planar structure and Fig. 28(c) is the energy band structure corresponding to this nanotube. The nanotube shown in Fig. 28(b) is formed by rolling the computed most stable $\mathrm{BC}_{2} \mathrm{~N}$ sheet geometry. Because of the anisotropic geometry of the $\mathrm{BC}_{2} \mathrm{~N}$ sheet, chiral structures are dominant. The chiral tubules have been found to be semiconducting (Miyamoto, Rubio, Cohen, and Louie, 1994). Either $p$ - or $n$-type semiconductors could be obtained by controlling the atomic stoichiometry of the sample (a composition $\mathrm{B}_{1-\delta} \mathrm{C}_{2+\delta} \mathrm{N}$ gives $\mathrm{C}$ impurities on $\mathrm{B}$ sites, resulting in electron carriers, while another composition, $\mathrm{BC}_{2+\delta} \mathrm{N}_{1-\delta}$, gives $\mathrm{C}$ impurities on $\mathrm{N}$ sites, resulting in hole carriers). Since the original sheets have anisotropic conductivity in the inplane directions, both $p$ - and $n$-type chiral tubules would be helical conductors (nanoscale coils) with potential technological application in nanodevices. However, there is still no experimental evidence for this tubular structure.

Band structure calculations of hypothetical tubule forms of hexagonal $\mathrm{BC}_{3}$ predicted that these tubes are as likely to form as carbon and $\mathrm{BN}$ nanotubes. The minimum energy gap predicted was about $0.2 \mathrm{eV}$ (Miyamoto, Rubio, Louie, and Cohen, 1994). Carroll et al. (1998) proposed that B substitution of CNTs leads to the formation of $\mathrm{BC}_{3}$ nanodomains inserted in the $1 \mathrm{D}$ nanotube structure, giving rise to an acceptor state $0.4 \mathrm{eV}$ below the Fermi level due to the realignment of this superstructure with the graphitic one. Fuentes et al. (2004) reported on a spectroscopic study of the highly B-doped C-SWNT using high-resolution nonspatially re- 

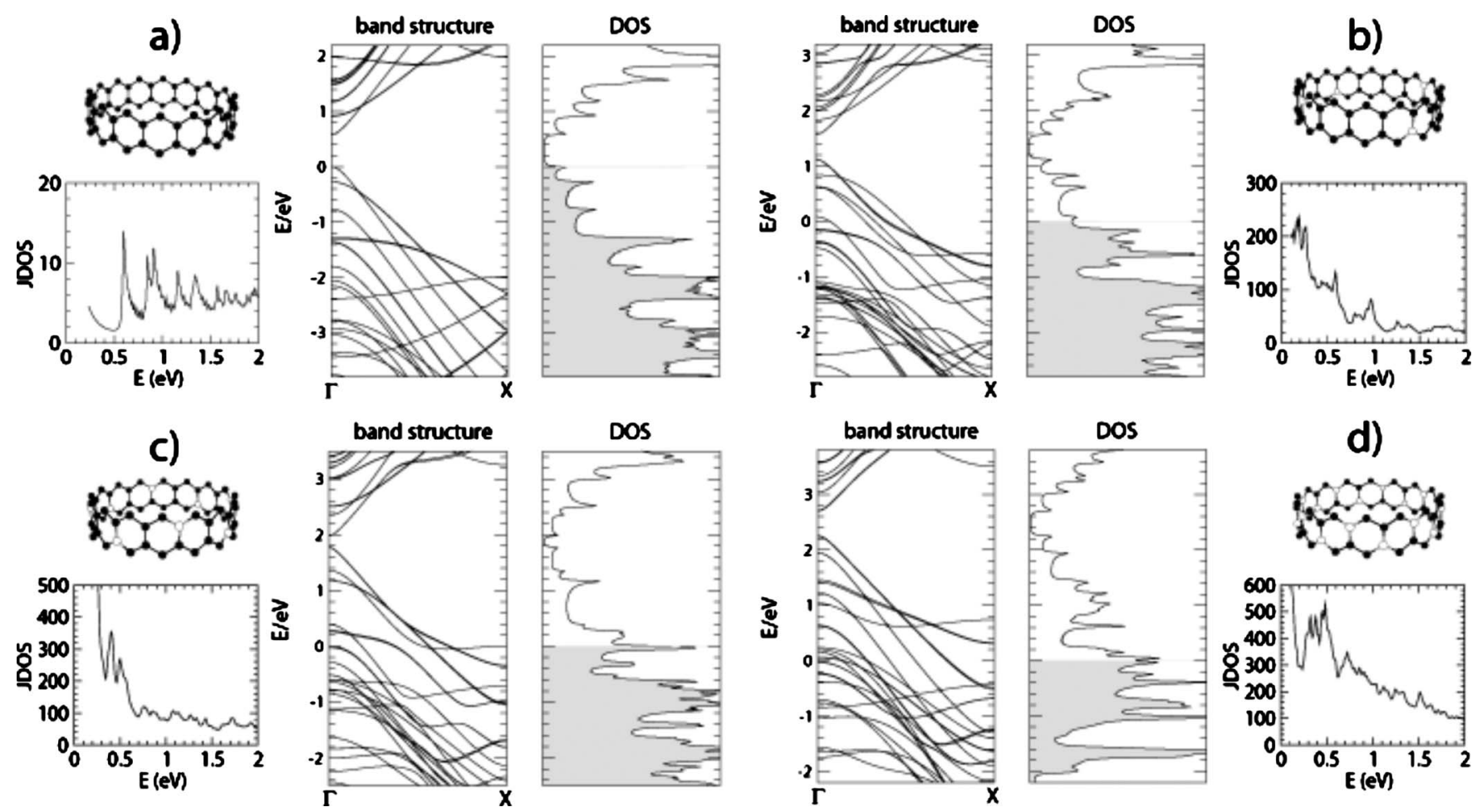

FIG. 27. The electronic configuration of B-doped nanotubes depends on the amount of heteroatomic incorporation in the nanotube structure. The band structure and DOS of a C(16,0) tube with (a) $0 \%$, (b) $6.25 \%$, (c) $12.5 \%$, and (d) $25 \%$ boron doping. Zero energy denotes the Fermi edge. Filled states are indicated by gray shading. From Fuentes et al., 2004.

solved EELS and optical spectroscopy. The spectroscopic response was then interpreted with the help of $a b$ initio band-structure calculations using DFT and the LDA. They suggested preferential doping of semiconducting C-SWNT and the formation of $\mathrm{BC}_{3}$ single-wall nanotubes in boron-substituted C-SWNT samples. How-
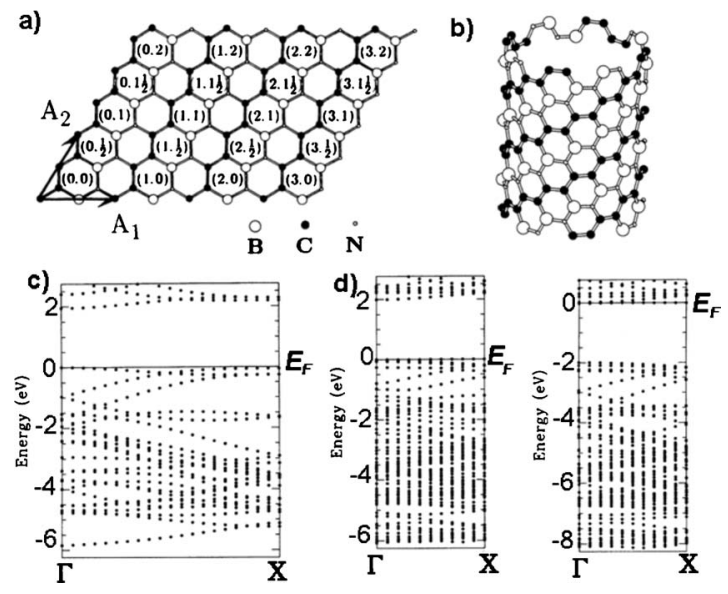

FIG. 28. $\mathrm{BC}_{2} \mathrm{~N}$ is one of the stoichiometries, which can lead to the formation of SWNTs. Here are shown one possible configuration of a $\mathrm{BC}_{2} \mathrm{~N}$ sheet (a) and the corresponding $\mathrm{BC}_{2} \mathrm{~N}(4,4)$ semiconducting nanotube obtained rolling the hexagonal stable structure (b). The lower panels correspond to the band structure of $(2,2) \mathrm{BC}_{2} \mathrm{~N}-\mathrm{SWNTs}$ (c) and the cases where a $\mathrm{C}$ atom is substituted as impurity on the $\mathrm{N}$ site (left, d) and the B site (right, d). Adapted from Miyamoto, Rubio, Cohen, and Louie, 1994. ever, a proof on the local basis is still required. Theoretically single-wall $\mathrm{BC}_{3}$ tubes are found to be semiconducting with a small $E_{g}(\sim 0.4 \mathrm{eV})$ that would disappear in the multilayer form (Fuentes et al., 2004). The interwall interaction for concentric tubes makes the conduction band overlap with the valence-band maximum ( $\sigma$ bands). Therefore, the concentric needle of $\mathrm{BC}_{3}$ tubes will have $\sigma$ conducting walls. It is also interesting to know how many walls are necessary to give rise to conductivity in concentric $\mathrm{BC}_{3}$ tubes: to look for a semiconductor-metal transition as a function of the number of concentric shells. Unfortunately, this question cannot be solved directly by conventional bandstructure calculations at present because of the geometrical difficulty of dealing with the concentric-tube incommensurate phases. Another characteristic of $\mathrm{BC}_{3}$ sheet and tubes is the two sharp peaks above $E_{F}$ in the DOS that stems from the $\pi$ and $\pi^{*}$ bands (see Fig. 29). The lower of the two peaks is below $E_{F}$ in the DOS of the $\mathrm{C}, \mathrm{BN}$, and $\mathrm{BC}_{2} \mathrm{~N}$ tubes. So the appearance of these two peaks can be used as evidence for the existence of $\mathrm{BC}_{3}$ tubes. The same double-peak structure should be observed in multiwall tubes, making this feature characteristic of the $\mathrm{BC}_{3}$ compounds.

The formation of $\mathrm{BC}_{3}$ SWNTs during the substitution process explains the higher local boron concentration observed on selected TEM spots and the remaining optical response of the pristine C-SWNT. Furthermore, different concentrations of $\mathrm{BC}_{3}$ tubes at different substitution levels also explain why the acceptor-induced 


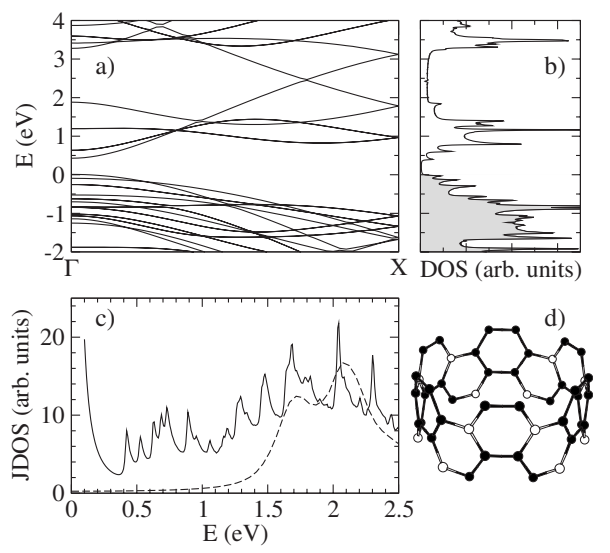

FIG. 29. $\mathrm{BC}_{3}$ nanotubes: (a) Electronic band structure of $\mathrm{BC}_{3}(3,3)$ nanotube (Fermi energy at $0 \mathrm{eV}$ ). (b) Density of states (filled states shaded with gray). (c) Joint density of states divided by $E^{2}$ (solid line) and calculated optical absorption (dashed line) of a perfect infinite $\mathrm{BC}_{3}(3,3)$ tube with light polarization along the tube axis. (d) Sketch of the unit cell. The black dots represent C atoms. From Fuentes et al., 2004.

absorption peak does not shift when the doping level is changed between $5 \%$ and $15 \%$. Since $\mathrm{BC}_{3}$ corresponds to $25 \% \mathrm{~B}$ substitution, and from the decrease of the C-SWNT intensity about $80 \%$ of the semiconducting C-SWNTs and within the experimental error non of the metallic tubes are doped, one ends up with an average doping level of about $13 \%$, in good agreement with the value derived from the core level excitation measurements and the optical results mentioned below.

\section{Electronic structure of BN-NTs}

As mentioned at the beginning of the paper, the electronic structure of $\mathrm{BN}$ nanotubes can be constructed from the electronic structure of a single-sheet $h-\mathrm{BN}$ as parent material by the zone-folding method. The most relevant features of $\mathrm{BN}$ nanotubes are their wide band gaps and the lack of dependence of the gap on the diameter of the tubes.

All BN nanotubes are found to be semiconducting materials. The band gaps are larger than $2 \mathrm{eV}$ for most tubes (Blase et al., 1994, 1995; Rubio et al., 1994). Depending on the helicity, the calculated band gap can be direct or indirect. In general, the larger the diameter of the nanotube the larger the band gap, with a saturation value corresponding to the calculated local-densityapproximation band gap of hexagonal BN (see Fig. 30). The higher ionicity of $\mathrm{BN}$ is important in explaining the electronic differences and similarities between these tubes and their carbon counterparts.

The wide band gap of BN-NTs implies that optical measurements should be undertaken in the UV range. Lauret et al. (2005) performed the first optical absorption experiments on partially purified films of $\mathrm{BN}-$ SWNTs and showed the existence of electronic transitions between 4.4 and $6.2 \mathrm{eV}$. These transitions not only confirmed the existence of a large band gap in these structures but were suggested to be associated with ex-

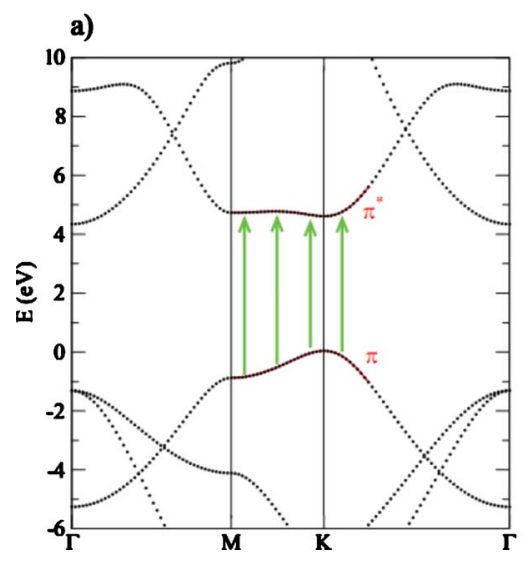

b)

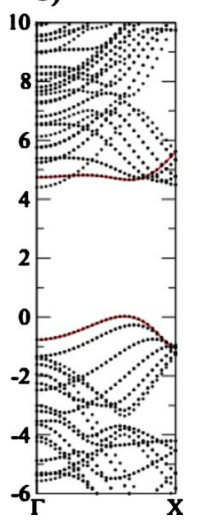

FIG. 30. (Color online) The band structure of a single sheet of $h$-BN (left) and the band structure of a $(6,6) \mathrm{BN}$.

citonic effects. As described, the study of the low-loss region $(<50 \mathrm{eV})$ in EELS provides direct access to the dielectric properties of the material. This spectroscopic information has been recorded on BN-SWNTs and BNMWNTs by Arenal et al. (2005) and Arenal, Stephan, et al. (2008). They demonstrated and confirmed that BN-NTs are insulators with a constant band gap $>5.5 \mathrm{eV}$, independent of the number of walls and the diameter.

Furthermore, it is worth mentioning that from the experimental point of view the direct measurement of the local density of states is not straightforward. Experiments with scanning tunneling spectroscopy on MWNTs have encountered a giant Stark effect which electrostatically interferes. In particular, the large gap and giant Stark effects have been shown to prevent one measuring the local density of state and the gap by scanning tunneling spectroscopy (Ishigami et al., 2005).

\section{OPTICAL PROPERTIES}

Absorption, photoluminescence, and Raman spectroscopy of the materials analyzed here will be discussed. As explained before, these techniques can provide a quick and reliable characterization of structure, morphology, and physical properties in general. The theory behind the methods and experimental results are explained in the following sections.

\section{A. N-doped C-SWNTs and $\mathrm{C}_{3} \mathrm{~N}_{4}$-SWNTs}

Lim et al. (2006) proposed that $\mathrm{CN}_{x}$-MWNTs can improve all-optical switching properties. However, these experimental studies are related to MW structures. In fact, there is limited experimental data regarding the optical properties of $\mathrm{CN}_{x}-\mathrm{SWNTs}$, and this can be related to the only recent availability of samples. Li et al. (2006) proposed that the presence of nitrogen significantly affects the emission properties of the $\mathrm{CN}_{x}$-NTs, causing a shift in the dominant emission to lower energies. However, this should be further studied, as the same authors 

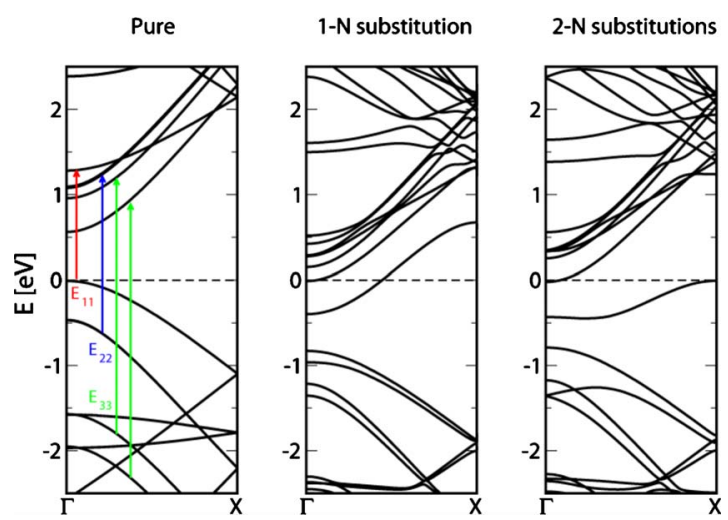

FIG. 31. (Color online) Electronic band structure of an $(8,0)$ nitrogen-doped C-NT with one and two N atom substitutions. From Gerber et al., 2009.

report these changes as possibly dependent on the bundling conditions.

Although it is still not possible to quantify the absolute amount of dopant in a sample with Raman spectroscopy alone the shift of the $G$ line, taking into account the modifications in the Kohn anomaly, has been reported to successfully reveal the relative $p$ - and $n$-type doping level. For N doping Villalpando-Paez et al. (2006) correlated the relative nitrogen content in the feedstock to the softening of the $G$ line and correlated it with the relative doping level and the change in the electrical conductivity. Gerber et al. (2009) focused on the influence of the $\mathrm{N}$ doping on the radial breathing mode in C-NTs via DFT calculations. They estimated the doping effects on the electronic band structure of the doped tubes, suggesting a downshift of the radial breathing mode (RBM), which should be dependent on the exact position of the dopant within the tube wall (see Fig. 31). They suggested that formation of N-N bonds is not favored for zigzag tubes and that a symmetric position of the dopant leads to only small downshifts of RBM frequencies. Recently Maciel et al. (2008) did this for the $2 D$ (also called $G^{\prime}$ ) peaks (see Fig. 18 and the corresponding reference). They found that the phonon at the $K$ point is hardened (it has a higher energy) in the presence of either $n$ - or $p$-type doping. This finding is reminiscent of previous studies on $n$-type intercalated and electrochemical doping of C-NTs, in which the Raman $G$ band, which corresponds to a zero-momentum ( $\Gamma$ point) optical phonon, was found to harden upon doping (Pichler et al., 2003; Kalbac et al., 2008, 2009).

In the above-mentioned study by Li, Xia, et al. (2006), they also reported optical measurements on SWNTs containing B, N, and C. They demonstrated that the incorporation of B and N in the network of C-SWNTs has an influence on their emission properties as well as on their structure.

Gracia and Kroll (2009) recently performed firstprinciples calculations on $\mathrm{C}_{3} \mathrm{~N}_{4}$-SWNTs, which are primarily made from heptazine heterocycles. They suggested that the electronic structure is isolated from structural modifications (i.e., hexagonal ordering). They described $\mathrm{C}_{3} \mathrm{~N}_{4}$-SWNTs as semiconductor materials with a stable band gap at about $3.0 \mathrm{eV}(410 \mathrm{~nm})$ with a strong absorption intensity expected between 4.3 and $4.4 \mathrm{eV}$ (280 and $290 \mathrm{~nm}$ ).

\section{B. B-doped C-SWNTs}

For $p$-type doping the same behavior for the $G$-line shift as for $\mathrm{FeCl}_{3}$ intercalation of C-SWNT (Kukovecz et al., 2002, 2003) response is predicted: a blueshift of the $G$ line. However, the few results on B-doped SWNT at low doping by McGuire et al. (2005) suggest that the $G$ line is hardly affected by the B doping. This is shown in Fig. 32. In the case of these samples, no local proof of doping has been reported so far, so the Raman studies on these tubes still cannot be considered as a pattern for other doped tubes. Maciel et al. (2008) also performed near-field measurements on individual B-doped SWNTs where they showed that the new split-off $2 D$ peak is upshifted with $p$-type doping. The authors present this as an unambiguous path to identify doping, but further studies are required in this respect.

Experimental data on optical absorption of B-doped NTs have also been reported by Fuentes et al. (2004) as shown in Fig. 33. Information about the dielectric function and hence the matrix-element-weighted joint DOS was retrieved from the normalized optical absorption of both B-doped and pristine C-SWNTs which are shown in Fig. 33 between 0.05 and $2.5 \mathrm{eV}$. In order to determine the relative peak intensities, the spectra were normalized and the background was subtracted. The spectrum of pristine C-SWNT is dominated by two strong absorptions at 0.75 and $1.35 \mathrm{eV}$ attributed to the two first allowed optical transitions between the vHss of the semiconducting C-SWNT modified by exciton formation (at an average tube diameter of $1.4 \mathrm{~nm}$ ), and a less intense feature at $1.93 \mathrm{eV}$ corresponding to the first allowed excitonic transition in metallic C-SWNTs. Essentially, the spectrum of B-doped C-SWNT shows the same absorption peaks as the pure C-SWNT but with an overall reduced intensity for the peaks originating from semiconducting tubes and with the same intensity in the case of the peak that corresponds to the metallic ones. In particular, the peak at $0.75 \mathrm{eV}$ is reduced to about $20 \%$ of its intensity. The fact that the metallic tubes are less affected by the substitution reaction than the semiconducting ones is consistent with calculations by Blase et al. (1999), who reported that the growth yield of metallic B-doped C-SWNTs is much lower than that of the semiconducting tubes. This fact suggests that the substitution method leads to a preferential B substitution of semiconducting tubes. Interestingly, a new absorption peak shows up at about $0.4 \mathrm{eV}$ in the case of the B-doped C-SWNT which was assigned by Wirtz and Rubio (2003) to an optical excitation from the valence band into a new unoccupied acceptorlike band induced by the boron substitution. The doping-dependent measurements by Borowiak-Palen et al. (2004) revealed that for a doping level of about 5 at. \% of B the position of this new ab- 

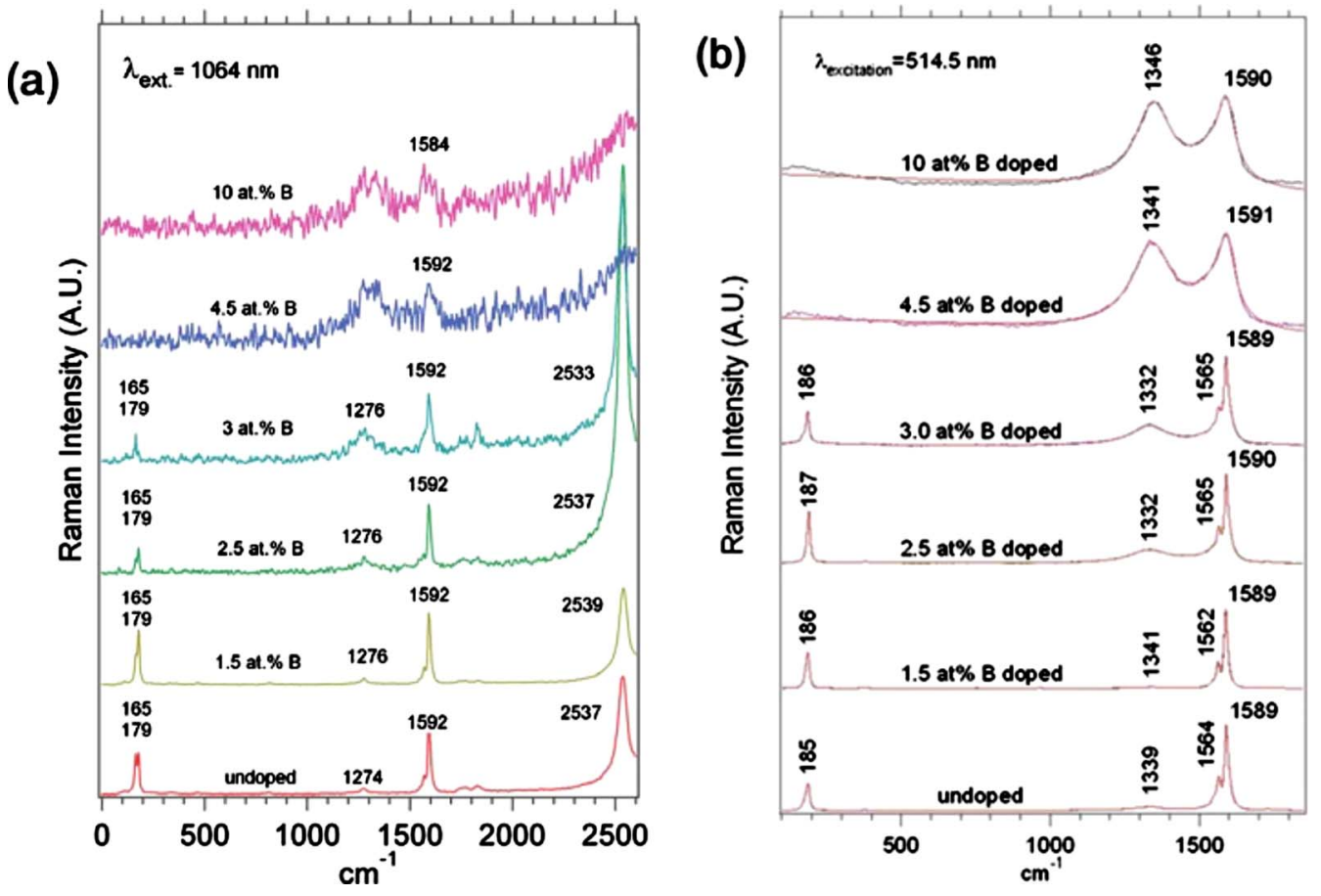

FIG. 32. (Color online) Raman spectroscopy study on B-doped C-SWNTs reported by McGuire et al., 2005. (a) First- and second-order room temperature Raman spectra of products generated from targets with the indicated B concentrations in the feedstock and excited using the $1064 \mathrm{~nm}$ excitation energy. (b) First-order room temperature Raman spectra of products generated from targets with the indicated B concentrations and excited using the $514 \mathrm{~nm}$ excitation energy. Adapted from McGuire et al., 2005 .

sorption peak remains basically unchanged, whereas the spectral weight is strongly reduced.

This idea is also further supported by resonant Raman spectroscopy as shown in Fig. 34. Whereas there is no modification of the $G$ lines in the region of the resonance of the metallic C-SWNT, a strong redshift is observed in the window of the semiconducting C-SWNT (with a maximum at $2.3 \mathrm{eV}$ ). This is different from the observations by McGuire et al. (2005) for low doping levels that there was hardly any change of the $G$ line. Since $p$-type doping usually leads to a blueshift of the

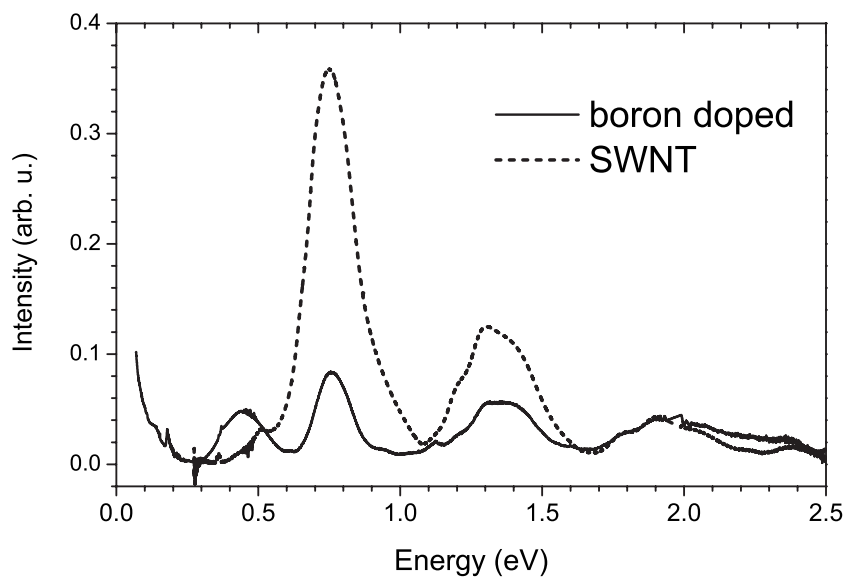

FIG. 33. Optical absorption spectra of boron-doped C-SWNT (solid) and pristine C-SWNT (dashed). From Fuentes et al., 2004. first-order tangential modes, this behavior has to have a different origin. One explanation is the preferential transformation of this part of the semiconducting $\mathrm{C}$-SWNT to other heteronanotubes such as $\mathrm{BC}_{3}$ nanotubes. This yields a loss of the resonance of these tubes and in Raman spectroscopy the preferential observation of the remaining thin-diameter SWCNT, in full agreement with the optical observations.

Hence, only a subset of tubes from the sample are doped (with a corresponding higher concentration)

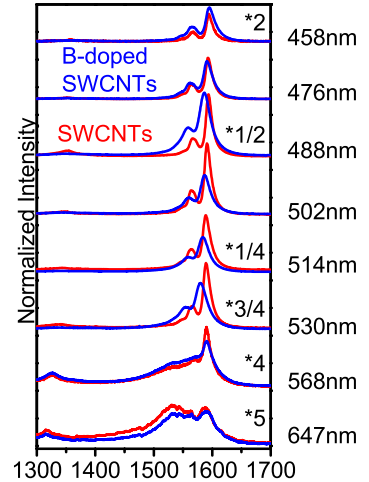

(a) Raman shift $\left(\mathrm{cm}^{-1}\right)$

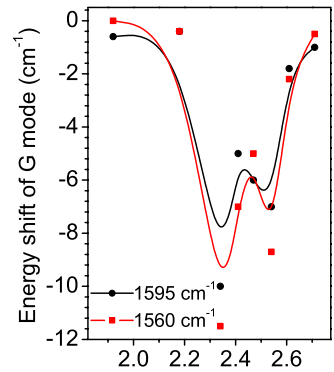

(b) Laser energy (eV)
FIG. 34. (Color online) The photoselective resonance Raman response for the $G$ modes in pristine and highly B-substituted (15 at. \%) SWNT (left). The relative softening of the $G$-line components as a function of laser energy and B doping is shown on the right panel. 

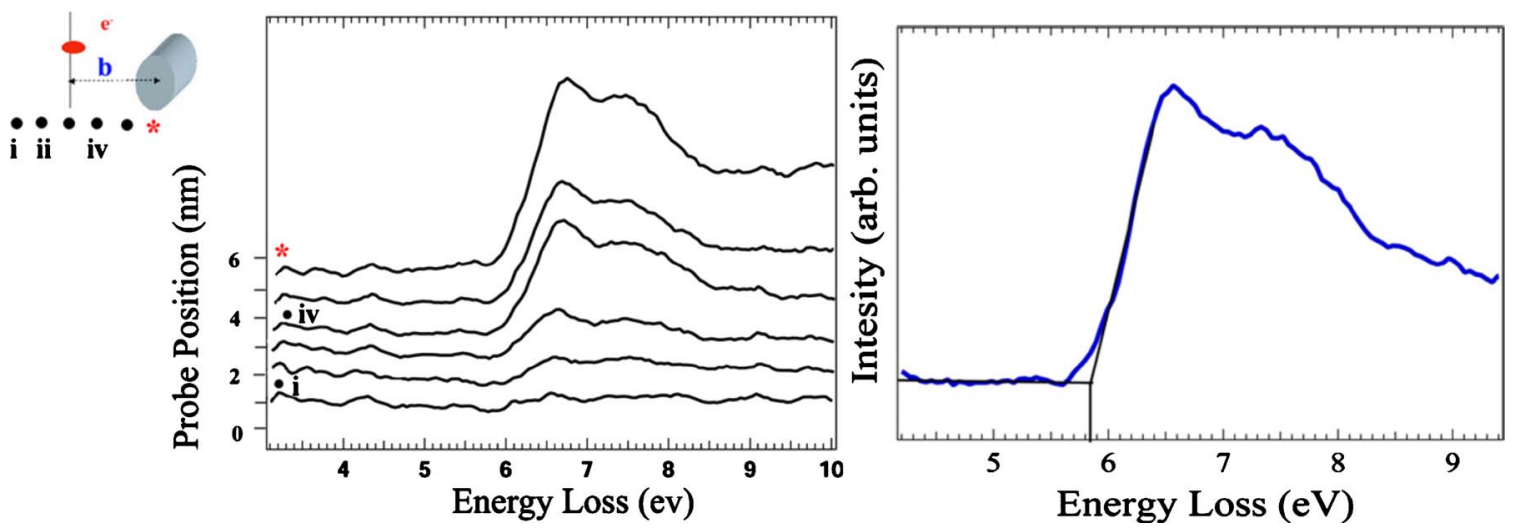

FIG. 35. (Color online) Spectrum line of a BN-SWNT with a measurement of the optical gap of a BN-NT from one of the loss spectra extracted from the left panel. Adapted from Arenal et al., 2005 and Arenal, Stephan, et al., 2008.

while the other tubes remain almost pure carbon tubes. This explains the stable peak positions in the spectra. The peaks at 0.7 and $1.4 \mathrm{eV}$ correspond to the vertical exitonic transitions between the first and second vHss in the pure semiconducting carbon tubes. In strongly B-doped samples, they should disappear because of the Fermi level lowering. However, the survival of a large fraction of pure carbon tubes explains the survival of these peaks and the decreasing intensity with increasing fraction of boron atoms in the sample. The unchanged intensity of the peak at $1.9 \mathrm{eV}$ is consistent with the assumption that metallic tubes are less susceptible to boron substitution (Blase et al., 1999). The formation of $\mathrm{BC}_{3}$ tubes in the samples ( $25 \%$ substitution) is consistent with the average degree of substitution (up to $15 \%$ ). The $\mathrm{BC}_{3}$ structure has been shown to be relatively stable, which is likely connected with the fact that these tubes are semiconducting with a gap between the occupied $\pi$ orbitals and the $\pi^{*}$ orbitals, that are unoccupied owing to the electron deficiency.

Nevertheless, further studies sensitive to the electronic properties of either metallic or semiconducting tubes should be carried out in order to understand the observed differences.

\section{C. $\mathrm{BC}_{2} \mathrm{~N}$ and $\mathrm{BC}_{3}$}

A nearly-free-electron state is seen for $\mathrm{BC}_{2} \mathrm{~N}$ sheets and nanotubes, but they are much higher in energy than BN nanotubes. Consequently, they do not play a significant role in the electronic properties. Barely anything is known of the optical properties for $\mathrm{BC}_{2} \mathrm{~N}$ so far.

For $\mathrm{BC}_{3}$, as mentioned, a uniform energy gap of about $1.8 \mathrm{eV}$ and an optically forbidden gap around $0.4-0.5 \mathrm{eV}$ is observed. As in BN-NT these gaps are hardly affected by the tube diameter or tube chirality. Therefore, the joint DOS [see Fig. 29(c)] displays a strong maximum about $0.4 \mathrm{eV}$. Accordingly, the first optically forbidden vertical transition is at $0.4 \mathrm{eV}$, whereas the first optically allowed transition is at about $1.8 \mathrm{eV}$ (similar to the case of hexagonal $\mathrm{BC}_{3}$ sheets) (Miyamoto, Rubio, Louie, and Cohen, 1994). Fuentes et al.
(2004) showed that this is reminiscent of the observed additional optical absorption peak in highly B-doped samples. The fact that an optically forbidden transition is observed was explained by symmetry breaking because of defects that are created during the substitution process in their samples. Therefore, because of the strong dependence of the band structure on the degree of substitution and the particular arrangement of the boron atoms, Fuentes et al. (2004) concluded that for highly boron-substituted SWNT the observed optical absorption spectra can be best explained with the assumption that only one particular kind of boron-substituted tube, $\mathrm{BC}_{3}$ nanotubes, is formed. This is a strong hint that at this high $\mathrm{B}$ doping level $\mathrm{BC}_{3}$ nanotubes are formed instead of B-doped C-SWNTs with random B dopant distribution. Nevertheless, further studies on individual highly B-doped samples should be carried out in order to finally confirm these observations.

\section{BN nanotubes}

Since BN nanotubes have a wide band gap independently of their geometry, then either multiphoton processes or uv light are necessary to excite an electron from the valence to the conduction band. A measurement of the optical gap of a single-wall BN-NT as measured by TEM-EELS is seen in Fig. 35.

The dielectric and optical properties of individual BNNTs have been investigated by EELS in the low-loss energy region using a dedicated STEM (Arenal et al., 2005; Arenal, Stephan, et al., 2008). These results have been interpreted using classical continuum dielectric theory. Using this model, these authors demonstrated the weak out-of-plane contribution to the dielectric response of these BN-NTs. The optical gap, which can be deduced from measurements that also allow the structure of the NT to be determined, is found to be $5.8 \pm 0.2 \mathrm{eV}$, which is close to that of $h$ - $\mathrm{BN}$, and is independent of diameter and structural configuration, in contrast to that of C-NTs.

The techniques available for optical characterization, at lower energy, infrared (ir) absorption probes the di- 


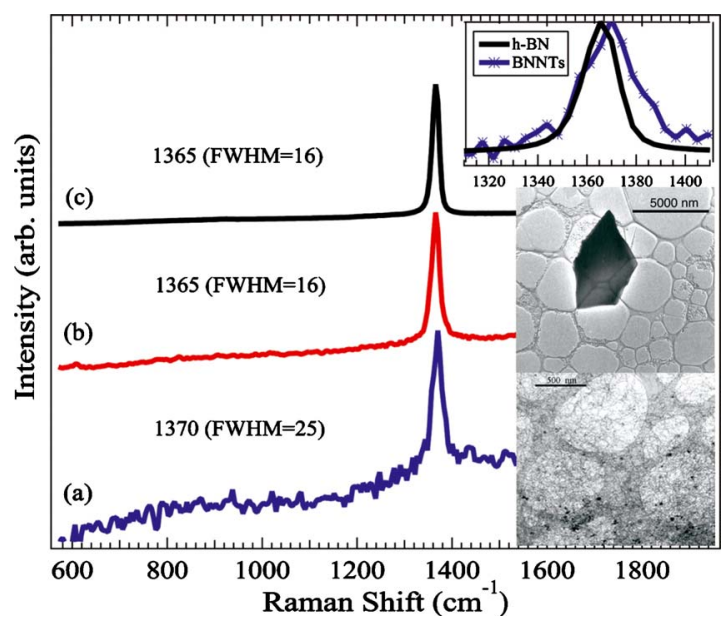

FIG. 36. (Color online) Raman spectra excited at $229 \mathrm{~nm}$ on (a) BN-NT-rich area in a standard TEM carbon grid, (b) a particle of $h$-BN on the same grid (see insets), and (c) highly crystalline powder $h$-BN. Adapted from Arenal, Ferrari, et al., 2006.

rect excitation of phonons and Raman spectroscopy probes the excitation of phonons by measuring the frequency shift in elastically scattered laser light.

In contrast to C-NTs, BN tubes have nonresonant Raman scattering in the visible spectral region because of their large band gap. It is then not surprising that careful attention should be given to the recorded spectra and the probable overlaps that could appear from resonance Raman scattering from contaminants (see Fig. 36 with the Raman spectra of BN nanotubes recorded with a laser of $229 \mathrm{~nm}$ ). From these results, Arenal, Ferrari, et al. (2006) showed that, working with a uv Raman excitation, preresonant conditions are obtained. These conditions allow the identification of the $A_{1}$ tangential mode (Wirtz et al., 2003) at $1370 \mathrm{~cm}^{-1}$. Furthermore, ab initio calculations showed that the increase in phonon frequency compared to that in bulk $h$-BN is related to a hardening of the $s p^{2}$ bonds in the BN tube case.

The efficiency of ir absorption is enhanced by the polarity of the material, and it gives rise to a much more pronounced ir spectrum than do CNTs, where the ir spectra have very little structure and can hardly be distinguished from the ir spectrum of graphite. In order to interpret such spectra, it is necessary to understand properly the phonon frequencies as a function of the tube diameter and chirality, together with the corresponding photoabsorption cross section. For zigzag and chiral nanotubes the set of infrared-active modes is only a subset of the Raman-active modes. In particular, the radial breathing mode is not only Raman but also infrared active. However, for armchair nanotubes, the sets of infrared- and Raman-active modes are disjoint. One more interesting feature to keep in mind is that, because of the large band gap of $h$-BN (>6 eV), optical absorption starts only in the uv regime; thus, it is difficult to measure. The theoretical understanding of these concepts is linked to the understanding of the luminescence properties which are also interesting; however.
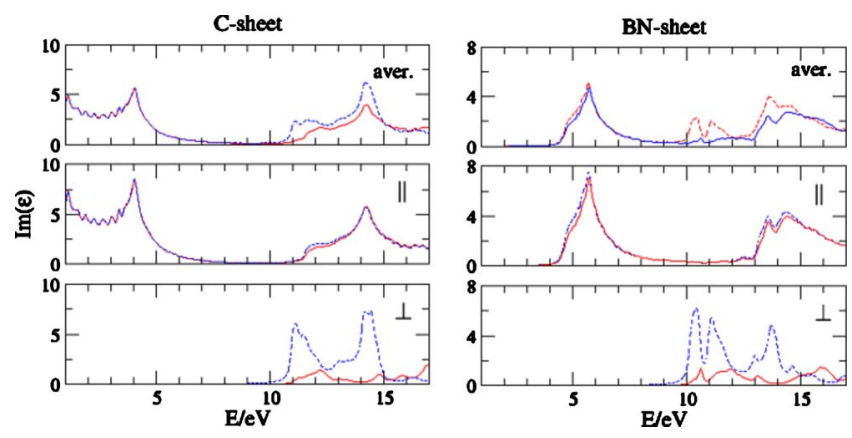

FIG. 37. (Color online) Random phase approximation spectra of graphene and a $h$-BN sheet, given by the parallel and perpendicular polarizations to the plane, as well as the averaged spectrum. Adapted from Marinopoulos et al., 2004.

The absorption cross section of an isolated nanotube is given by the imaginary part of its polarizability per unit length. To first order, absorption of a photon of energy $\hbar \omega$ is commonly explained through the vertical excitation of an electron from a state $\left|n_{v}\right\rangle$ with energy $E_{v}$ in the valence band to a conduction band state $\left|n_{c}\right\rangle$ of energy $E_{c}$,

$$
\operatorname{Im}[\alpha(\omega)] \propto \sum_{n_{v}, n_{c}}\left|\left\langle n_{v}|D| n_{c}\right\rangle\right|^{2} \delta\left(E_{c}-E_{v}-\hbar \omega\right),
$$

where $D$ is the dipole operator that promotes the valence-to-conduction excitation upon light absorption. This equation is the random phase approximation (RPA), which is also known as the independent-particle picture of absorption.

In order to understand how an absorption spectrum is constructed one has to look at the RPA spectrum of a single sheet of $h$-BN and of the $(6,6)$ nanotube. Figure 37 shows the RPA absorption spectrum of the sheet. In the low-energy regime $(<10 \mathrm{eV})$ and for light-polarization parallel to the sheet, the dipole matrix element in Eq. (1) selects only the transition from the $\pi$ to the $\pi^{*}$ band (see Fig. 30). The onset of the spectrum is at $4.5 \mathrm{eV}$ which corresponds to the direct gap of the sheet at $K$. The peak at $5.7 \mathrm{eV}$ corresponds to the $M$ point where the $\pi$-to- $\pi^{*}$ bands display saddle points and the DOS has a maximum.

If the light is polarized perpendicularly to the sheet (Fig. 37), the $\pi$-to- $\pi^{*}$ transitions are dipole forbidden. Optical absorption is caused by $\sigma$-to- $\pi^{*}$ and $\pi$-to- $\sigma^{*}$ transitions, and the onset of the RPA spectrum is only at about $10 \mathrm{eV}$. Figure 37 also shows the effect of depolarization. The dashed line shows the spectrum calculated with Eq. (1). The external field polarizes the charge distribution of the sheet, creating a layer of dipoles. This dipole layer, in turn, leads to an induced electric field that is directed opposite to the external field. The absorption spectrum must therefore be calculated selfconsistently. (Mathematically, this corresponds to taking into account off-diagonal elements of the dielectric tensor in reciprocal space.) The resulting spectrum (solid line in Fig. 37) is strongly reduced in oscillator strength in the energy range below $15 \mathrm{eV}$. For parallel polarization, the depolarization effects have only a minor influ- 

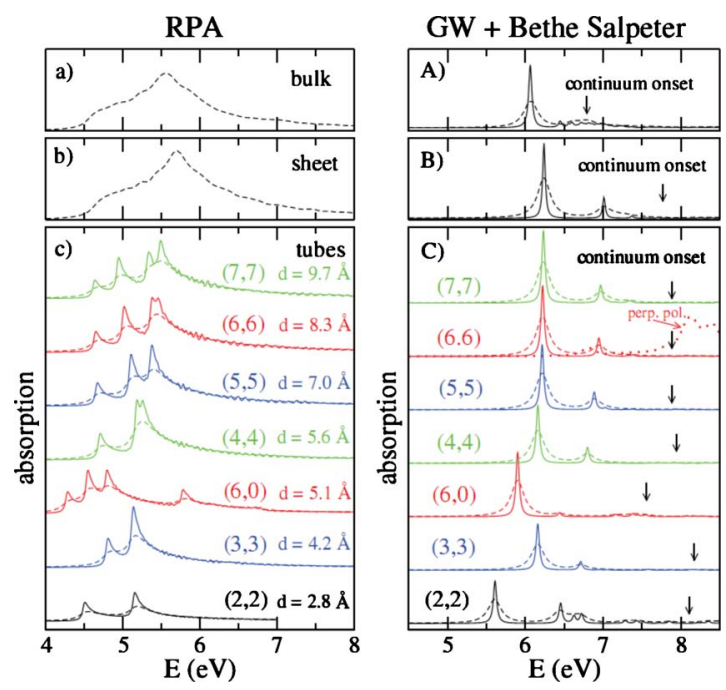

FIG. 38. (Color online) RPA absorption spectra of (a) bulk hexagonal $\mathrm{BN}$, (b) a single sheet of hexagonal $\mathrm{BN}$, and (c) six different $\mathrm{BN}$ tubes with increasing diameter $d$. Solid lines are calculated with a Lorentzian broadening of $0.025 \mathrm{eV}$ and dashed lines with a broadening of $0.1 \mathrm{eV}$. From Wirtz et al., 2006.

ence. The direction-averaged spectrum in Fig. 37 is dominated by the contribution from the parallel polarization. Figure 38 shows the RPA spectra of bulk $h$-BN, of a single sheet of $h$-BN, and of different BN nanotubes with diameters ranging from $2.8 \AA$ for the purely hypothetical $\mathrm{BN}(2,2)$ tube to $9.7 \AA$ for the $\mathrm{BN}(7,7)$ tube, which is at the lower border of the range of experimentally produced tubes. The light polarization is set parallel to the planes or tube axis, because as discussed depolarization effects strongly suppress the absorption in the perpendicular direction. For the bulk and single sheet the spectra are almost indistinguishable. This is because of the relatively weak interaction between neighboring sheets in the bulk phase. Since the bands of the tubes can be constructed from the sheet via the zone-folding procedure, the RPA spectra of the tubes display transitions at the same energies as in the sheet. With increasing diameter, the shape of the tube spectra converges rapidly toward the sheet spectrum.

\section{Influence of the correlation effects of the absorption spectrum}

A photon is absorbed by exciting an electron from the valence band to the conduction band. For a quantitative prediction of the spectrum, one needs to know the exact size of the band gap. Furthermore, it is necessary to take into account that the excited electron may interact with the hole that is left behind in the valence band. These studies are done using many-body perturbation theory with a self-energy formalism in which electron correlations are treated on the level of the $G W$ approximation while the electron-hole attraction is dealt with by means of a static Bethe-Salpeter equation.

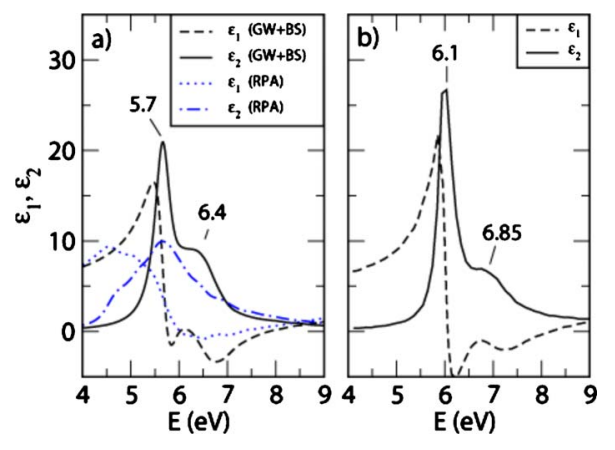

FIG. 39. (Color online) Real $\left(\epsilon_{1}\right)$ and imaginary $\left(\epsilon_{1}\right)$ parts of the dielectric function of $h$-BN calculated in the $G W+\mathrm{BSE}$ approach and in the RPA (a). Experimental data from EELS, where $\epsilon_{1}$ and $\epsilon_{2}$ are calculated from the loss function via a Kramers-Kronig transformation. The calculations include a Lorentzian broadening of $0.2 \mathrm{eV}$ (FWHM) in order to represent the estimated experimental broadening. The light polarization is considered as parallel to the BN layers (b). From Wirtz et al., 2005.

\section{Excitonic effects}

In addition to the electron-electron $(e-e)$ interaction, the electron-hole $(e-h)$ interaction can play an important role in the quantitative description of absorption spectra. The excited electron in the conduction band and the hole left behind in the valence band interact through an attractive Coulomb potential. As in the case of the $e-e$ interaction, the attractive $e-h$ potential is screened through the inverse dielectric function. In many largeband-gap materials, the $e-h$ attraction leads to the formation of bound excitons, i.e., discrete states in the band gap. On a qualitative level, bound excitons can be compared with bound states of the hydrogen atom.

Before discussing the effect of the $e-e$ and $e-h$ interactions on the spectra of nanotubes, it is necessary to consider the absorption spectrum of bulk $h$-BN because $h$-BN is the reference material for BN-SWNTs and because for bulk $h$-BN sufficient experimental data exist to judge the validity of the combined approach of $G W$ for the $e$-e interaction and the Bethe-Salpeter (BS) equation for the $e-h$ correlation.

Results for the energy-dependent dielectric function of $h$-BN (light polarization parallel to the layers) are shown in Fig. 39(a) and compared with the experimental data from EELS (Tarrio and Schnatterly, 1989) in Fig. 39(b). The dash-dotted line shows the RPA absorption spectrum, which is in agreement with RPA calculations (Strinati, 1984; Xu and Ching, 1991; Guo and Lin, 2005). The broad peak with a maximum at $5.6 \mathrm{eV}$ is entirely due to the continuum of interband transitions between the $\pi$ and $\pi^{*}$ bands (see Fig. 40). The calculated $G W$ + BS absorption spectrum displays a double-peak structure with the main peak at $5.7 \mathrm{eV}$ and a second peak at $6.4 \mathrm{eV}$. The shape of the spectrum is entirely different from the RPA spectrum: the first peak is due to a strongly bound exciton (Arnaud et al., 2006), and the second peak contains contributions from higher excitons 


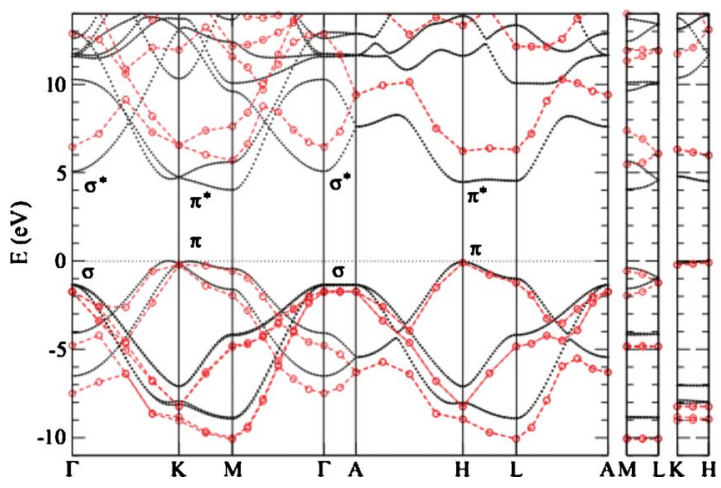

FIG. 40. (Color online) Band structure of bulk $h$-BN: LDA and $G W$ approximation. From Wirtz et al., 2005.

and from the onset of the continuum of interband transitions. The similarity between the RPA and $G W+\mathrm{BS}$ spectra stems exclusively from the strong broadening employed in the calculation. The main peaks in the two spectra are at about the same position because of an almost cancellation between the band-gap widening from the $G W$ approximation and the redshift of oscillator strength from excitonic effects. A comparison of this with Fig. 39(b) shows that the shape of the $G W+\mathrm{BS}$ spectrum is in much better agreement with experiment than the RPA spectrum. This underlines the importance of excitonic effects in $h$-BN. The influence of excitonic effects becomes even more pronounced when we compare the real part of $\epsilon$. Only the $G W+\mathrm{BS}$ calculation can reproduce qualitatively the shape of the experimental real part of the dielectric response function.

Even if the exciton confinement to one layer in bulk $h$-BN is not perfect, it leads to a considerable enhancement of the exciton binding energy with respect to nonlayered materials. In the inset in Fig. 41(c), an image of

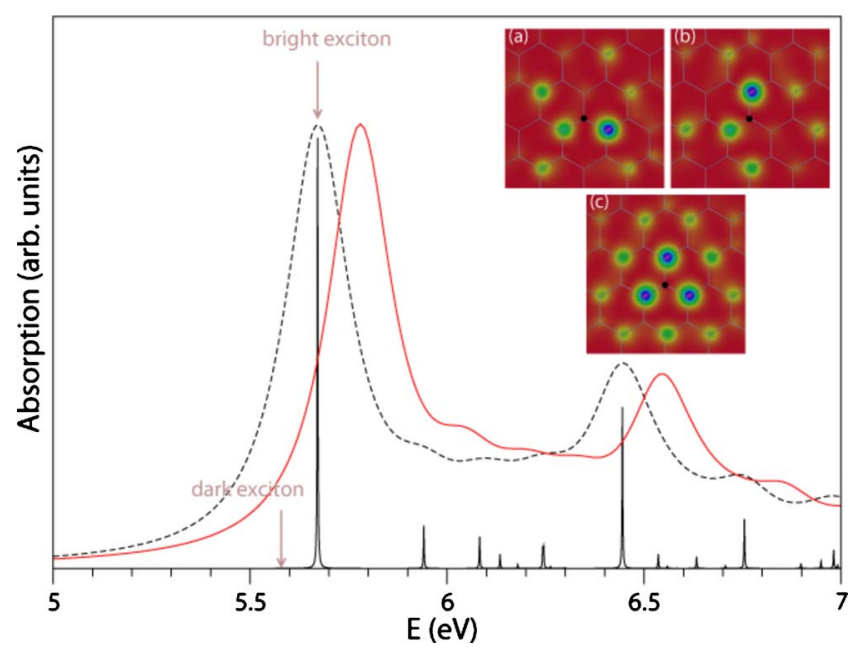

FIG. 41. (Color online) Optical absorption spectrum of $h$-BN. An estimated experimental broadening of $0.1 \mathrm{eV}$ calculated with YAMBO (black dashed line) and with VASP (solid line). Inset: Two-dimensional projections of the probability density of degenerate exciton states with $\lambda=$ (a) 3 and (b) 4. From Wirtz et al., 2005. the excitonic wave function is shown. The wave-function plot represents the probability density to find the excited electron if the hole is at a given position. Clearly, the probability is enhanced around the boron atoms [the lowest unoccupied molecular orbital (LUMO) being a superposition of boron $p_{z}$ orbitals]. Furthermore, the probability density is confined to within a few atomic distances. In Fig. 41, about $\sim 90 \mathrm{meV}$ below the dominant excitonic peak there is a "dark" bound exciton. This exciton cannot be directly excited through absorption of a photon, but it may play a role in luminescence. Both the dark and bright excitons are doubly degenerate states. The electron densities of each of the two states that contribute to the bright exciton are not rotationally symmetric [Figs. 41(a) and 41(b)] but by addition of the densities of the two states, the expected threefold rotation symmetry is recovered [Fig. 41(c)].

If the symmetry of the perfect crystal is broken, the degeneracy of the excitons is lifted and the dark exciton acquires some oscillator strength. Figure 41 shows calculations with two different $G W+\mathrm{BS}$ codes. The small difference (of the order of $0.1 \mathrm{eV}$ ) in the absolute positions of the spectra most likely arise from the different pseudopotentials that play a role in the $G W$ correction to the band gap. Both spectra have in common that they are about $0.3 \mathrm{eV}$, which is relatively low in comparison with the various experimental data (Tarrio and Schnatterly, 1989; Lauret et al., 2005). One reason for this (small) mismatch may be that the $G W$ approximation is only the first-order correction to the band gap. Higherorder corrections may enlarge the theoretical band gap even further and thus blueshift the spectrum. Another explanation is the effect of phonon renormalization on the absorption spectrum. Theoretical calculations are usually performed at zero temperature. Only recently were the first $a b$ initio calculations of excitonic effects including the exciton-phonon coupling achieved (Marini, 2008). This allowed the first calculation of absorption spectra at finite temperature. For bulk $h$-BN at room temperature the dominant excitonic peak has been shown to be at $5.98 \mathrm{eV}$, in excellent agreement with the experimental data (Lauret et al., 2005).

After the discovery of C-NTs in 1991, for several years the optical spectra were discussed only in the independent-particle picture. In 1997, the presence of excitons in carbon tubes was predicted by Ando (1997). Since $G W+$ BS calculations are expensive and since the unit cell of nanotubes comprises more than 20 atoms, it was only in 2004 that the first ab initio excitonic spectra of carbon tubes were published (Spataru et al., 2004, 2008). The excitonic nature of the spectra was confirmed in numerous experiments such as Wang et al. (2005) and Ma et al. (2006). Since BN-NTs have a much larger band gap than semiconducting CNTs, it can be expected that excitonic effects are even more pronounced than in CNTs. This has indeed been confirmed by $G W+\mathrm{BS}$ calculations (Park et al., 2006; Wirtz et al., 2006). Because of the lower dimensionality (and lower screening of the $e-e$ interaction) in both the single sheet and isolated BN 

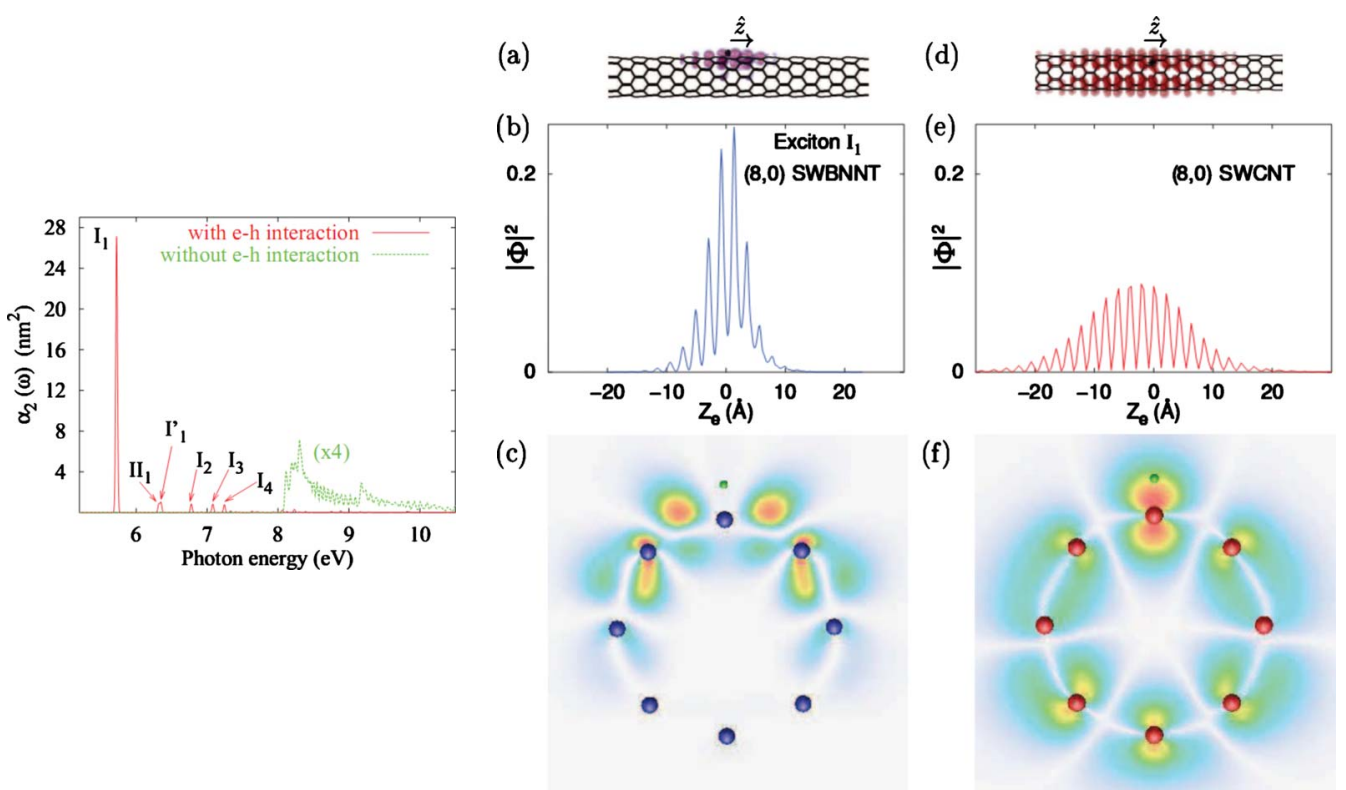

(f)

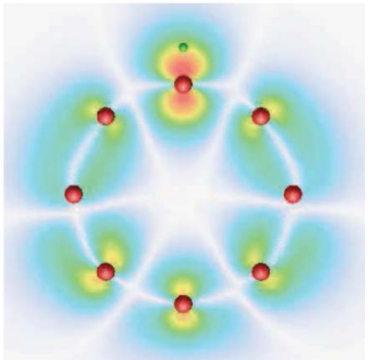

FIG. 42. (Color online) Absorption spectra of $(0,8)$ BN-SWNTs are shown on the left side graphic. The spectra are broadened with a Gaussian of $0.0125 \mathrm{eV}$. On the right are depicted the wave function of the lowest energy bright exciton and isosurface plots of the electron probability distribution of a $(8,0)$ BN-SWNT (a)-(c) and of a C-SWNT (d)-(f). From Park et al., 2006.

nanotubes, the $G W$ band gap correction (with respect to the DFT-LDA band structure) is strongly enhanced compared to that in bulk $h$-BN: $3.6 \mathrm{eV}$ for the single sheet (Kresse and Furthmuller, 1996) and $3.25 \mathrm{eV}$ for the $(8,0)$ tube (Park et al., 2006) as compared to $1.8 \mathrm{eV}$ for bulk $h$-BN.

The left panel of Fig. 41 shows the absorption spectrum of the $\mathrm{BN}(8,0)$ tube with and without $e$ - $h$ interaction (in both cases, $G W$ corrections to the band gap are included). Without $e-h$ interaction, the continuum of band-to-band transitions would start above $8 \mathrm{eV}$. A strong excitonic binding energy of $2.3 \mathrm{eV}$ leads, however, to a first absorption peak at $5.72 \mathrm{eV}$. As in the case of bulk $h$-BN, the first absorption peak comprises most of the oscillator strength of the entire absorption spectrum. The wave function of the $5.72 \mathrm{eV}$ exciton is shown in Figs. 42(a)-42(c) and compared to the wave-function of the lowest bright exciton in an $(8,0)$ carbon nanotube [Figs. 42(d)-42(f)]. The hole is located above a chosen $\mathrm{N}$ (C) atom on one side of the tube. While the exciton in the carbon tube is delocalized around the whole tube circumference, the exciton in the $\mathrm{BN}$ tube is localized on the side where the hole is located. Along the tube axis also the exciton in the $\mathrm{BN}$ tube is much more localized than the one in the carbon tube. This is in line with the much higher excitonic binding energy in $\mathrm{BN}$ tubes than in $\mathrm{C}$ tubes. Figure 38 shows the excitonic absorption spectra for a series of BN nanotubes compared with the spectra of the sheet and bulk BN (Wirtz et al., 2006). On the right panel of Fig. 38 is shown how the RPA spectra are modified once excitonic (electron-hole) effects are included at the $G W+\mathrm{BS}$ level. Now it is worth comparing the spectra of the single sheet and of bulk $h$-BN. With increasing diameter, the shape of the tube spectra converges rapidly toward the sheet spectrum, which in turn is not very different from the spectrum of the bulk. The rapid convergence as a function of tube diameter toward the sheet spectrum can be understood from Fig. 42. Since the exciton is not delocalized around the circumference but localized within a few nearest neighbors' distance, it "sees" a locally flat environment and thus behaves as an exciton in the flat sheet. We note that there is a chirality dependence of the optical spectra but it is visible only for the smallest-diameter tubes. The spectra of the armchair tubes converge much faster to the $2 \mathrm{D}$ case than those of the zigzag tubes. Many-body effects in carbon nanotubes have been found to be quite different (Chang et al., 2004; Spataru et al., 2004): binding energies and quasiparticle shifts are much smaller, and the extension of the excitonic wave function (several nanometers) is larger than the typical tube circumference. Thus, excitonic binding energies vary strongly with the diameter. Excitons in C-NTs are one-dimensional objects, i.e., squeezed in the circumferential direction. The strongly localized nature of the exciton in BN structures restricts the appearance of one-dimensional confinement effects to small diameter tubes, i.e., tubes for which the extension of the excitonic wave function is comparable to the nanotube circumference. As the experimental tubes have diameters around $1.4 \mathrm{~nm}$, the $1 \mathrm{D}$ nature of the tubes cannot be observed and only the $2 \mathrm{D}$ nature of the local exciton environment (tube surface) controls the optical activity. We note that dimensionality effects in the electronic properties of BN nanostructures would be more visible in other spectroscopic measurements such as photoemission spectroscopy, where we mainly map the quasiparticle spectra, and this (like the exciton binding itself) is sensitive to the change in screening going from the tube to the sheet to bulk hexagonal BN. In particular, the quasiparticle band gap will 


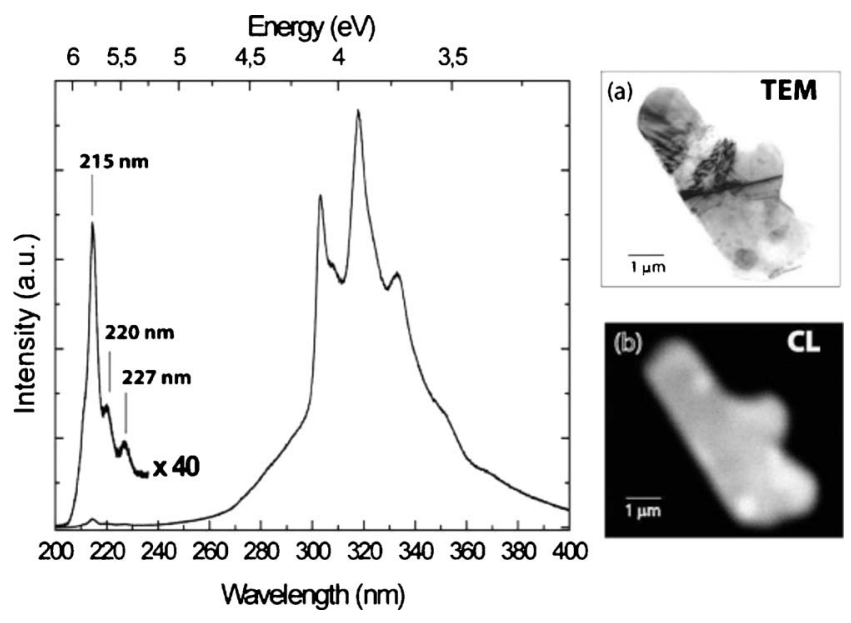

FIG. 43. Cathodoluminescence spectrum of $h$-BN crystallite at $T=100 \mathrm{~K}$. (a) TEM image of the $h$-BN crystallite and (b) polychromatic CL image. From Jaffrennou et al., 2007.

vary strongly with dimensionality (opening as the dimensionality is reduced).

\section{Luminescence}

Recently the observed high luminescence yield in bulk $h$-BN crystals has raised the interest in $\mathrm{BN}$ compounds as potential candidates for uv light-emitting materials. The main luminescence peak (for pure $h$-BN crystals) was measured at $215 \mathrm{~nm}(5.77 \mathrm{eV})$. It is ascribed to the radiative decay of the lowest-lying exciton. In the electronic structure community, this exciton is called a "bound exciton" because it corresponds to an electron that is bound to a hole in the valence band. Thus the energy of this state lies below the range of "free" conduction-band electrons (see above discussion on optical absorption). In contrast, the luminescence spectroscopy community tends to call this exciton a "free exciton" because it is independent of structural defects of the material. For the remainder of this section, we use the notation of luminescence spectroscopy. In comparison to the main absorption peak, which is located between 6 and $6.1 \mathrm{eV}$, the luminescence peak experiences a strong Stokes shift toward lower energy. We note, however, that recent photoluminescence excitation (PLE) spectra (i.e., measurement of the luminescence intensity as a function of the exciting laser energy) locate the absorption peak corresponding to the free exciton rather at $5.81 \mathrm{eV}$ (Museur and Kanaev, 2008).

This yields only a very small Stokes shift of the luminescence peak $(5.77 \mathrm{eV})$. At low temperature $(<100 \mathrm{~K})$, additional luminescence peaks at $220 \mathrm{~nm}$ $(5.64 \mathrm{eV})$ and $227 \mathrm{~nm}(5.46 \mathrm{eV})$ have been observed (see Fig. 43). Jaffrennou et al. (2007) found that luminescence at the latter two wavelengths occurs at the grain boundaries and around dislocations. They ascribed the two peaks to excitons that are bound to structural defects and thus have a higher binding energy than the free exciton (and thus a lower position in the luminescence spectrum). At very low temperature $(8 \mathrm{~K})$, Museur and
Kanaev (2008) found an additional bound exciton line at $5.56 \mathrm{eV}$ and a line at $5.3 \mathrm{eV}$ that they ascribed to a transition between filled acceptor-band donor states. Luminescence peaks in $h$-BN have also been observed in the energy region of $4 \mathrm{eV}$ (Taylor et al., 1994; Silly et al., 2007; Han et al., 2008) (see Fig. 43). They have a much lower intensity than the high-energy peaks and are explained by the presence of deep level impurities, probably due to $\mathrm{C}$ or $\mathrm{O}$ atoms. Indeed, when $h$-BN crystals were grown under ultraclean conditions, the $4 \mathrm{eV}$ energy band disappeared from the luminescence spectra. The main peak displays several phonon replica on the low-energy side. The proof that those peaks are really due to electron-phonon coupling was given by Han et al. (2008) who showed that the splitting between the peaks, i.e., the vibrational frequency, changes as a function of the boron isotope. The nature of the deep level impurity is not clear. However, calculations indicate the stability of $\mathrm{O}$ in substitution for $\mathrm{N}$ atoms. Vacancies and other impurities like C could give rise to deep levels as well.

The assignment of the defect-related peaks observed in luminescence requires more detailed theoretical work. However, it seems that all luminescence features observed below the main absorption peak are defectmediated excitations, i.e., excitons bound to structural defects or transitions from or to acceptor or donor levels. Luminescence on multiwalled BN-NT samples has been observed by several groups. Wu et al. (2004) performed photoluminescence on multiwall BN tubes. They detected only the deep level impurity peaks around $4 \mathrm{eV}$. The zero-phonon line at $4.02 \mathrm{eV}$ was falsely ascribed to the direct band gap. Others also detected the excitonic bands in the luminescence of multiwalled BNNTs (Jaffrennou et al., 2007). Bound excitonic peaks were observed at 5.49 and $5.34 \mathrm{eV}$ (Jaffrennou et al., 2007), i.e., at somewhat lower energy than in bulk $h$-BN $(5.77 \mathrm{eV})$. This difference was tentatively explained: trapping of excitons on crystalline defects appears to be a major phenomenon. In the tubes, dislocations and stacking faults along the walls may lead to a stronger trapping of excitons than in $h$-BN and to an absence of the free-exciton line at $5.77 \mathrm{eV}$. The stronger trapping leads to slight redshift of the bound excitonic lines with respect to the corresponding lines in the PL spectra of bulk $h$-BN. From this discussion it is a logical next step to try to build lasing or optoelectronic devices with $\mathrm{BN}$ nanotubes. In this respect, it is interesting to know if the optical properties can be tuned in a controlled way. It has been shown theoretically that the band gap of BNNTs can be reduced by application of an electric field perpendicular to the tube axis. This decrease of the gap is due to the Stark effect, i.e., the charge densities of the top of the valence band (Taylor et al., 1994) and bottom of the conduction band become spatially separated on opposite sides of the tube. Even though the gap is reduced considerably, the effect of an electric field on the optical absorption spectrum was found to be less pronounced (for light polarization parallel to the tube axis, which gives the dominant contribution to the averaged 

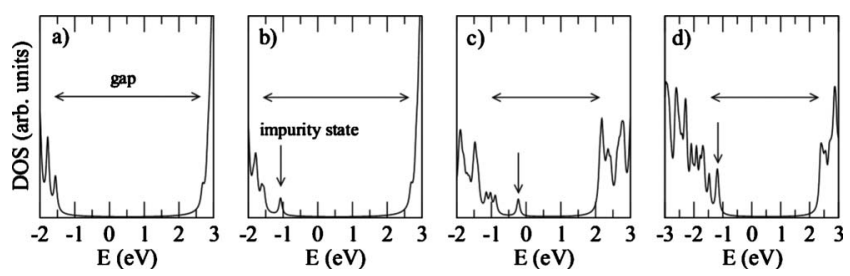

FIG. 44. DOS of (a) a pure $(9,9) \mathrm{BN}$ nanotube, (b) a tube with a carbon impurity atom, (c) with a $\mathrm{C}$ atom and a perpendicular $E$ field of $0.2 \mathrm{~V} / \AA$, and (d) with a $\mathrm{C}$ atom on the opposite side and an $E$ field of $0.2 \mathrm{~V} / \AA$ The light polarization is parallel to the tube axis.

light-scattering cross section). This situation, however, may change for luminescence spectra. Peaks around $4 \mathrm{eV}$ in the luminescence spectra are related to defect levels (possible candidates discussed above are vacancies and oxygen or carbon substitutions). As an example, we calculated the influence of the electric field on the acceptor level that is due to the replacement of a nitrogen atom by a carbon atom. [The calculations have been performed in a large supercell as shown in Fig. 44, the DOS of the pure $(9,9)$ BN-NT, calculated within DFT-LDA.] Addition of the carbon impurity in Fig. 44(b) introduces an impurity level at about $0.5 \mathrm{eV}$ above the valenceband edge. Depending on the orientation of the carbon impurity with respect to the direction of the electric field, this impurity level can move up or down with respect to the valence- and conduction-band edges [Figs. 44(c) and 44(d)]. Photoluminescence may involve transitions from the conduction band to the impurity level and from the impurity level to the valence band. Defectmediated luminescence spectra may thus be more sensitive to the influence of an electric field than the spectra of pure BN-NTs.

\section{ASSESSMENT OF THE APPLICATION POTENTIAL}

One feature of heteronanotubes of $\mathrm{B}, \mathrm{C}$, and $\mathrm{N}$ is the fact that, depending on their stoichiometry, their behavior opens a wide range of application possibilities. However, we emphasize that most reported studies involving applications are constrained to theoretical predictions, because of the still scarce availability of clean samples of these types of heteronanotubes.

C-SWNTs doped with B and N can be used as tunable semiconductors if doped in low amounts. For this reason, a separation of nanotubes with high and low doping needs to be considered carefully during production.

In general, the chemical modification of either sidewalls or end caps of the nanotubes has been shown to be important for chemical reactivity. Thus, heteronanotubes of $\mathrm{B}, \mathrm{C}$, and $\mathrm{N}$ are good candidates for applications that require wall functionalization of the nanotube material. There are several experimental reports for doped C-SWNTs, especially regarding the case of N doping. For instance, Villalpando-Paez et al. (2006) showed that N-doped C-SWNTs exhibit improved conductivity upon optimal incorporation of $\mathrm{N}$ in the substi-
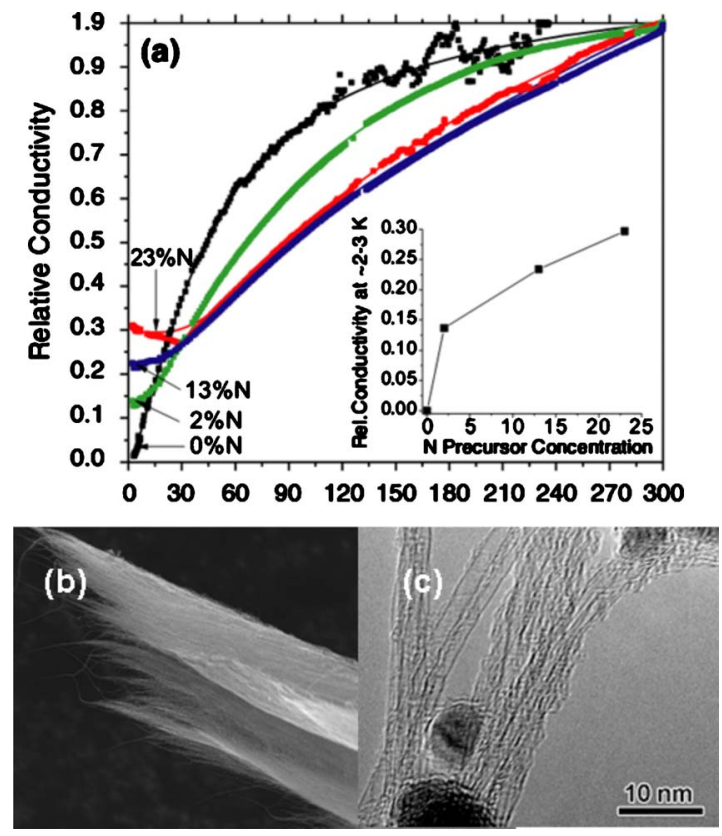

FIG. 45. (Color online) N-doped C-SWNTs have exhibited improved conductivity upon optimal incorporation of $\mathrm{N}$ in substitutional configuration. Adapted from Villalpando-Paez et al., 2006.

tutional configuration (see Fig. 45). Considering applications in bulk quantities, among the most important innovations in a new generation of composites could be the use of C-NTs, functionalized NTs, and heteronanotubes of $\mathrm{B}, \mathrm{C}$, and $\mathrm{N}$ as promising components in polymer, ceramic, and metal composites. Not only do the outstanding mechanical properties that NTs exhibit make them attractive alternative reinforcing fillers, but also their electronic behavior when embedded in matrixes is of great interest. An interesting advantage in comparison to pristine C-NT materials, where the difficulties of developing bulk scale metallicity separation have not been overcome, makes the use of BN-NTs and $\mathrm{BC}_{3}$-NTs a promising alternative in composite applications, considering that they are either insulators or semiconductors showing a large chemical and thermal stability.

Boron nitride nanotubes have been theoretically investigated but the experimental reports, which are recent, are focused on multiwalled BN nanotube solubilization (Maguer et al., 2007). The insulating characteristics of BN-NTs made them ideal candidates for use as electrically insulating nanocables, which in turn could be used in nanoscale complex circuitry. High luminescence yields in bulk $h$-BN crystal have raised interest in $\mathrm{BN}$ compounds as potential candidates for uv light-emitting materials, and BN-NTs are not an exception.

Recently Liu et al. (2008) reported results of studies on the sheet resistance and optical transmission of thin films of boron-doped C-SWNTs with 1-3 at. \% substitutional boron found in the SWNT bundles via EELS (see Fig. 46). Boron doping was found to downshift the posi- 


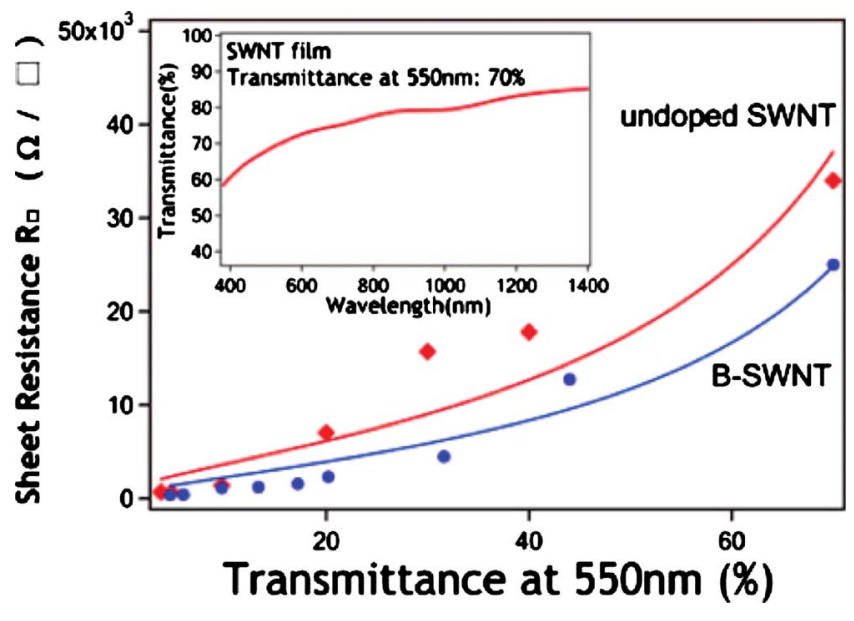

FIG. 46. (Color online) Use of boron-doped nanotubes as transparent conducting films. The transmittance (at $550 \mathrm{~nm}$ ) and sheet resistance of B-doped SWNT and undoped P-SWNT films. From Liu et al., 2008.

tions of the optical absorption bands associated with the van Hove singularities $\left(E_{11}^{s}, E_{22}^{s}\right.$, and $\left.E_{11}^{m}\right)$ by $30 \mathrm{meV}$ relative to their positions in acid-treated and annealed SWNTs. These results suggest that B doping does not significantly affect the optical transmittance in the visible region but it lowers the sheet resistance by $30 \%$ relative to pristine SWNT films from the same batch. They therefore proposed B doping of C-SWNT as a better approach to touch-screen technology.

Hydrogen storage has been widely suggested as a promising application of nanotubes because of their cylindrical and hollow geometry, large specific surfaces, and a highly porous structure. Work with C-SWNTs hints at better performance of the semiconducing C-SWNTs for hydrogen adsorption but this still needs to be proved. Furthermore, Zhou et al. (2004b) studied the atomic and molecular adsorption of hydrogen on B- and $\mathrm{N}$-doped C-SWNTs by DFT calculations. They suggested that $\mathrm{B}$ doping increases the hydrogen atomic adsorption energies in both zigzag and armchair nanotubes. On the other hand, $\mathrm{N}$ doping apparently decreases the hydrogen atomic adsorption energies. Zhang et al. (2007) studied the mechanisms for hydrogen dissociative adsorption and diffusion on $\mathrm{N}$-doped $(8,0)$ C-NTs in detail by carrying out DFT calculations. They found that nitrogen doping considerably alters the catalytic effects of the carbon nanotube for hydrogen dissociative adsorption. This adsorption of hydrogen on the carbon nanotube is greatly enhanced, with the barrier substantially reduced to $\sim 0.9 \mathrm{eV}$. Further studies have suggested that the introduction of substitutional carbon impurity increases the reactivity of $\mathrm{BN}$ nanotube toward molecular oxygen, and stable $\mathrm{O}_{2}$ chemisorption states exist on both carbon-substituted nitrogen (C-N) and carbon-substituted boron (C-B) defect sites. Rossato et al. (2008) did a first-principles study of the adsorption of atomic and molecular hydrogen on $\mathrm{BC}_{2} \mathrm{~N}-\mathrm{NTs}$ that showed promising results.

It has been suggested that B-doped C-SWNTs, consid- ered as hole-doped material, could play the role of dilute magnetic semiconductors when incorporated into field-effect transistor devices (Owens, 2007). Calculations of the lithium absorption energies and electronic structures of boron-doped C-SWNTs suggest that enhanced $\mathrm{Li}$ absorption capability in the B-doped C-SWNT should be expected (Zhou et al., 2004a). Field emission of B- (Charlier et al., 2002) and N- (Ahn et al., 2006) doped nanotubes also has promising perspectives. Recent studies showed that boron-doped C-NTs are good candidates for direct electrochemistry of redoxactive enzymes and the construction of related enzyme biosensors (Deng et al., 2008a, 2008b). In addition, Li, $\mathrm{Xia}$, et al. (2006) proposed the use of $\mathrm{CN}_{x}$-NTs as saturable optical absorber devices. Futhermore, $\mathrm{BC}_{2} \mathrm{~N}$ also exhibits great potential for applications. This is suggested, for instance, in the early work by Watanabe et al. (1996), who reported that layered $\mathrm{BC}_{2} \mathrm{~N}$ is a semiconductor with a direct band gap that emits visible light.

\section{SUMMARY AND FUTURE PERSPECTIVES}

In this review we have given insight into the field of hybrid and functionalized single-walled nanotubes containing $\mathrm{B}, \mathrm{C}$, and $\mathrm{N}$ atoms. The generation of defects and dopants within these tubular structures has been discussed, targeting their great importance for emerging technologies.

We have covered the theoretical concepts in depth, as well as the experimental achievements from carbon nanotubes with low $\mathrm{B}$ or $\mathrm{N}$ doping to the new physics behind boron nitride nanotubes. In order to give a realistic approach to their applicability we have devoted special attention to reviewing the bulk and local characterization tools required to study these materials, their suitability, and limitations. The theoretical approaches regarding the physical and chemical properties are objectively analyzed versus the materials currently available. In the various sections of the paper, we review how several experimental and theoretical investigations must focus toward a controllable modification of the crystalline nanotube properties by placement of atomic-scale imperfections in a controlled manner. All the unique structural, electronic, mechanical, electromechanical, and chemical properties of these heteronanotubes have been linked to intensive research efforts worldwide because understanding these properties and exploring their potential applications must be the main motivating force in this area. So far, extensive efforts have been devoted to synthesizing the high-purity and defect-free SWCNTs and to control their diameter and chiralities. Theoretical and experimental work has also been focused on the relationship between the atomic structures and the electronic transport properties of nanotubes, as well as electron-electron and electron-phonon interactions. Furthermore, it has been shown that the uniformity of the energy gap in $\mathrm{BC}_{2}-\mathrm{SWNTs}$ and $\mathrm{BN}-\mathrm{SWNTs}$ is also promising for nanoelectronics and optoelectronics (Blase et al., 1997; Rubio, 1997a, 1997b).

Finally, we remark that there is still much interesting 
research to be done on topological defects as a consequence of doping or formation of the structures described in this review. Heterostructures formed by joining nanotubes of different chirality may also show unique quasi-one-dimensional transport properties and still need to be intensively explored. Once the synthesis of these structures is achieved in a controlled manner, it will be possible to make structures such as electronic switches.

\section{ACKNOWLEDGMENTS}

This work was supported by the Austrian Science Fund through Project No. FWF P21333-20. P.A. and T.P. acknowledge the financial support of the following projects DFG PI 440/3/4/5 and the fruitful discussions and collaboration with E. Borowiak-Palen, S. Daothong, J. Fink, G. Fuentes, E. Kauppinen, M. Knupfer, C. Kramberger, X. Liu, J. Parjanne, T. Susi, and M. Terrones. A.R. acknowledges funding by the Spanish MEC (Grant No. FIS-65702-C02-01), "Grupos Consolidados UPV/EHU del Gobierno Vasco" (Grant No. IT-319-07), and the European Community through e-I3 ETSF project (Contract No. 211956), DNA-NANODEVICES (Grant No. IST-2006-029192), and NANO-ERA CHEMISTRY. We acknowledge support by the Barcelona Supercomputing Center, "Red Española de Supercomputacion" and SGIker ARINA (UPV/EHU). A.R. also acknowledges his fruitful collaborations on inorganic nanotubes over the years with $\mathrm{C}$. Attacalite, P. Bernier, M. Cohen, C. Goze, M. Gruning, S. G. Louie, A. Marini, Y. Miyamoto, J. Serrano, L. Wirtz, and A. Zettl. A.L. and R.A. acknowledge the financial support of the European Commission under the 6 Framework Programme STREP Project BNC Tubes (Contract No. NMp4-CT-2006-033350), the "Agence National de la Recherche"-France (A.N.R) and their collaboration with B. Attal-Tretout, L. Bresson, J. L. Cochon, C. Colliex, N. Dorval, F. Ducastelle, S. Enouz-Védrenne, A. C. Ferrari, M. Glerup, P. Jaffrennou, M. Kociak, S. Lefrant, H. Lin, A. Maguer, J. Y. Mevellec, D. Pigache, S. Reich, J. Serrano, O. Stephan, D. Taverna, M. Tence, and L. Wirtz. We acknowledge the valuable contributions and discussion with Dr. S. Roche, Dr. V. Kristic, Dr. X. Liu, Dr. M. Terones, and Dr. L. Wirtz during the preparation of this manuscript.

\section{REFERENCES}

Ahn, H., K. Lee, D. Kim, and S. Han, 2006, Appl. Phys. Lett. 88, 093122.

Alibart, F., O. Durand-Drouhina, M. Benlahsena, S. Muhlb, S. Elizabeth, C. E. Rodil, and L. Escobar-Alarcon, 2008, Appl. Surf. Sci. 254, 5564.

Ando, T., 1997, J. Phys. Soc. Jpn. 66, 1066.

Arenal, R., X. Blase, and A. Loiseau, 2010, Adv. Phys. 59, 101. Arenal, R., F. de la Pena, O. Stephan, M. Walls, M. Tence, A. Loiseau, and C. Colliex, 2008, Ultramicroscopy 109, 32.

Arenal, R., A. Ferrari, S. Reich, L. Wirtz, J. Mevellec, S. Lefrant, A. Rubio, and A. Loiseau, 2006, Nano Lett. 6, 1812.
Arenal, R., M. Kociak, A. Loiseau, and D. J. Miller, 2006, Appl. Phys. Lett. 89, 073104.

Arenal, R., M. Kociak, and N. J. Zaluzec, 2007, Appl. Phys. Lett. 90, 204105.

Arenal, R., O. Stephan, J.-L. Cochon, and A. Loiseau, 2007, J. Am. Chem. Soc. 129, 16183.

Arenal, R., O. Stephan, M. Kociak, D. Taverna, A. Loiseau, and C. Colliex, 2005, Phys. Rev. Lett. 95, 127601.

Arenal, R., O. Stephan, M. Kociak, D. Taverna, A. Loiseau, and C. Colliex, 2008, Microsc. Microanal. 14, 274.

Arnaud, B., S. Lebègue, P. Rabiller, and M. Alouani, 2006, Phys. Rev. Lett. 96, 026402.

Arnold, M., A. Green, J. Hulvat, S. Stupp, and M. Hersam, 2006, Nat. Nanotechnol. 1, 60.

Ashcroft, N., and N. Mermin, 1976, Solid State Physics (Holt, Rinehart and Winston, New York).

Avriller, R., S. Roche, F. Triozon, X. Blase, and S. Latil, 2007, Mod. Phys. Lett. B 21, 1955.

Ayala, P., R. Arenal, M. H. Rümmeli, A. Rubio, and T. Pichler, 2010, Carbon 48, 575.

Ayala, P., F. L. Freire, Jr., M. H. Rümmeli, A. Grueneis, and T. Pichler, 2007, Phys. Status Solidi B 244, 4051.

Ayala, P., A. Grüneis, T. Gemming, B. Büchner, M. Rümmeli, D. Grimm, J. Schumann, R. Kaltofen, F. L. Freire, Jr., and T. Pichler, 2007, Chem. Mater. 19, 6131.

Ayala, P., A. Grüneis, T. Gemming, D. Grimm, C. Kramberger, M. Rümmeli, F. Freire, Jr., H. Kuzmany, R. Pfeiffer, A. Barreiro, B. Büchner, and T. Pichler, 2007, J. Phys. Chem. C 111, 2879.

Ayala, P., A. Grüneis, C. Kramberger, M. Rümmeli, M. Solórzano, F. Freire, Jr., and T. Pichler, 2007, J. Chem. Phys. 127, 184709.

Ayala, P., Y. Miyata, K. De Blauwe, H. Shiozawa, R. Silva, R. Follath, H. Kataura, C. Kramberger, and T. Pichler, 2009, Phys. Rev. B 80, 205427.

Ayala, P., W. Plank, A. Grüneis, E. Kauppinen, M. Rümmeli, H. Kuzmany, and T. Pichler, 2008, J. Mater. Chem. 18, 5676. Ayala, P., J. Reppert, M. Grosbosch, M. Knupfer, T. Pichler, and A. M. Rao, 2010, Appl. Phys. Lett. 96, 183110.

Bachilo, S., L. Balzano, J. Herrera, F. Pompeo, D. Resasco, and R. Weisman, 2003, J. Am. Chem. Soc. 125, 11186.

Belz, T., et al., 1998, Carbon 36, 731.

Biró, L., J. Gyulai, P. Lambin, J. Nagy, S. Lazarescu, G. Mark, A. Fonseca, P. Surjan, Z. Szekeres, P. Thiry, and A. Lucas, 1998, Carbon 36, 689.

Blase, X., J.-C. Charlier, A. D. Vita, and R. Car, 1997, Appl. Phys. Lett. 70, 197.

Blase, X., J. C. Charlier, A. De Vita, R. Car, P. Redlich, M. Terrones, W. K. Hsu, H. Terrones, D. L. Carroll, and P. M. Ajayan, 1999, Phys. Rev. Lett. 83, 5078.

Blase, X., A. De Vita, J.-C. Charlier, and R. Car, 1998, Phys. Rev. Lett. 80, 1666.

Blase, X., A. Rubio, S. Louie, and M. Cohen, 1994, Europhys. Lett. 28, 335.

Blase, X., A. Rubio, S. G. Louie, and M. L. Cohen, 1995, Phys. Rev. B 51, 6868.

Borowiak-Palen, E., T. Pichler, G. Fuentes, A. Graff, R. Kalenczuk, M. Knupfer, and J. Fink, 2003, Chem. Phys. Lett. 378, 516.

Borowiak-Palen, E., T. Pichler, G. Fuentes, A. Graff, R. Kalenczuk, M. Knupfer, and J. Fink, 2004, Carbon 42, 1123.

Cao, C., F. Huang, C. Cao, J. Li, and H. Zhu, 2004, Chem. Mater. 16, 5213. 
Carroll, D. L., P. Redlich, X. Blase, J. C. Charlier, S. Curran, P. M. Ajayan, S. Roth, and M. Ruhle, 1998, Phys. Rev. Lett. 81, 2332.

Caudal, N., A. M. Saitta, M. Lazzeri, and F. Mauri, 2007, Phys. Rev. B 75, 115423.

Cermignani, W., T. Paulson, C. Onneby, and C. Pantalo, 1995, Carbon 33, 367.

Chang, E., G. Bussi, A. Ruini, and E. Molinari, 2004, Phys. Rev. Lett. 92, 196401.

Charlier, J., M. Terrones, M. Baxendale, V. Meunier, T. Zacharia, N. Rupesinghe, W. Hsu, N. Grobert, H. Terrones, and G. Amaratunga, 2002, Nano Lett. 2, 1191.

Charlier, J.-C., X. Blase, and S. Roche, 2007, Rev. Mod. Phys. 79, 677.

Chen, G. G., C. A. Furtado, S. Bandow, S. Iijima, and P. C. Eklund, 2005, Phys. Rev. B 71, 045408.

Chen, Y., L. T. Chadderton, J. F. Gerald, and J. S. Williams, 1999, Appl. Phys. Lett. 74, 2960.

Chen, Y., J. F. Gerald, J. S. Williams, and S. Bulcock, 1999, Chem. Phys. Lett. 299, 260.

Choi, Y., et al., 2003, Nano Lett. 3, 839.

Chopra, N., R. J. Luyken, K. Cherrey, V. Crespi, M. Cohen, S. Louie, and A. Zettl, 1995, Science 269, 966.

Cumings, J., and A. Zettl, 2000, Chem. Phys. Lett. 316, 211.

Czerw, R., et al., 2001, Nano Lett. 1, 457.

Das, A., A. K. Sood, A. Govindaraj, A. M. Saitta, M. Lazzeri, F. Mauri, and C. N. R. Rao, 2007, Phys. Rev. Lett. 99, 136803.

Deng, C., J. Chen, X. Chen, C. Mao, L. Nie, and S. Yao, 2008a, Biosens. Bioelectron. 23, 1272.

Deng, C., J. Chen, X. Chen, C. Mao, Z. Nie, and S. Yao, 2008b, Electrochem. Commun. 10, 907.

Dreizler, R., and E. Gross, 1990, Density Functional Theory, an Approach to the Quantum Many Body Problem (Springer, Berlin).

Dresselhaus, M., G. Dresselhaus, and P. Avouris, 2001, Carbon Nanotubes: Synthesis, Structure, Properties, and Applications (Springer-Verlag, Berlin).

Dresselhaus, M. S., G. Dresselhaus, and R. Saito, 1995, Carbon 33, 883, nanotubes.

Droppa, R., Jr., P. Hammer, A. Carvalho, and F. Alvarez, 2002, J. Non-Cryst. Solids 874, 299.

Egerton, R. F., 1996, Electron Energy-Loss Spectroscopy in the Electron Microscope, 2nd ed. (Plenum, New York).

Elias, A. L., P. Ayala, A. Zamudio, M. Grobosch, E. CruzSilva, J. M. Romo-Herrera, J. Campos, H. Terrones, T. Pichler, and M. Terrones, 2010, J. Nanosci. Nanotechnol. 10, 3959.

Enouz, S., O. Stephan, J.-L. Cochon, C. Colliex, and A. Loiseau, 2007, Nano Lett. 7, 1856.

Enouz, S., O. Stephan, M. Glerup, J. L. Cochon, C. Colliex, and A. Loiseau, 2006, Phys. Status Solidi B 243, 3246.

Enouz-Védrenne, S., O. Stephan, M. Glerup, J.-L. Cochon, C. Colliex, and A. Loiseau, 2008, J. Phys. Chem. C 112, 16422.

Ewels, C., and M. Glerup, 2005, J. Nanosci. Nanotechnol. 5, 1345.

Fink, J., 1989, Adv. Electron. Electron Phys. 75, 121.

Fiolhais, C., F. Nogueira, and M. Marques, 2003, A Primer in Density Functional Theory, Lecture Notes in Physics No. 620 (Springer, Berlin).

Franceschini, D., C. Achete, and F. Freire, Jr., 1992, Appl. Phys. Lett. 60, 3229.

Fuentes, G. G., E. Borowiak-Palen, M. Knupfer, T. Pichler, J. Fink, L. Wirtz, and A. Rubio, 2004, Phys. Rev. B 69, 245403.
Fuentes, G. G., E. Borowiak-Palen, T. Pichler, X. Liu, A. Graff, G. Behr, R. J. Kalenczuk, M. Knupfer, and J. Fink, 2003, Phys. Rev. B 67, 035429.

Gai, P., O. Stephan, K. McGuire, A. Rao, M. Dresselhaus, G. Dresselhaus, and C. Colliex, 2004, J. Mater. Chem. 14, 669.

Gammon, W., O. Kraft, A. Reilly, and B. C. Holloway, 2003, Carbon 41, 1917.

Gerber, I. C., P. Puech, A. Gannouni, and W. Bacsa, 2009, Phys. Rev. B 79, 075423.

Glerup, M., J. Steinmetz, D. Samaille, O. Stephan, S. Enouz, A. Loiseau, S. Roth, and P. Bernier, 2004, Chem. Phys. Lett. 387, 193.

Golberg, D., Y. Bando, L. Burgeois, K. Kurashima, and T. Sato, 2000, Carbon 38, 2017.

Golberg, D., Y. Bando, W. Han, K. Kurashima, and T. Sato, 1999, Chem. Phys. Lett. 308, 337.

Golberg, D., Y. Bando, K. Kurashima, and T. Sato, 2000, Chem. Phys. Lett. 323, 185.

Golberg, D., Y. Bando, C. Tang, and C. Zhi, 2007, Adv. Mater. (Weinheim, Ger.) 19, 2413.

Golberg, D., A. Rode, Y. Bando, M. Mitome, E. Gamaly, and B. Luther-Davies, 2003, Diamond Relat. Mater. 12, 1269.

Gracia, J., and P. Kroll, 2009, J. Mater. Chem. 19, 3020.

Guo, G. Y., and J. C. Lin, 2005, Phys. Rev. B 71, 165402.

Haider, M., S. Uhlemann, E. Schwan, H. Rose, B. Kabius, and K. Urban, 1998, Nature (London) 392, 768.

Hamada, N., S. I. Sawada, and A. Oshiyama, 1992, Phys. Rev. Lett. 68, 1579.

Hamilton, E., S. Dolan, C. M. Mann, H. Colijn, C. McDonald, and S. Shore, 1993, Science 260, 659.

Han, W., Y. Bando, K. Kurashima, and T. Sato, 1998, Appl. Phys. Lett. 73, 3085.

Han, W.-Q., H.-G. Yu, C. Zhi, J. Wang, Z. Liu, T. Sekiguchi, and Y. Bando, 2008, Nano Lett. 8, 491.

Hassanien, A., M. Tokumoto, T. Shimizu, and H. Tokumoto, 2004, Thin Solid Films 464-465, 338.

Hellgren, N., J. Guo, C. Sathe, A. Gui, J. Nordgren, Y. Luo, H. Agren, and J.-E. Sundgren, 2001, Appl. Phys. Lett. 79, 4348. Hellgren, N., M. P. Johansson, E. Broitman, L. Hultman, and J.-E. Sundgren, 1999, Phys. Rev. B 59, 5162.

Hernández, E., P. Ordejon, I. Boustani, A. Rubio, and J. Alonso, 2000, J. Chem. Phys. 113, 3814.

Ichimura, K., M. Osawa, K. Nomura, H. Kataura, Y. Maniwa, S. Suziki, and Y. Achiba, 2002, Physica B 323, 230.

Iijima, S., 1991, Nature (London) 56, 354.

Iijima, S., and T. Ichihashi, 1993, Nature (London) 363, 603.

Ishigami, M., J. D. Sau, S. Aloni, M. L. Cohen, and A. Zettl, 2005, Phys. Rev. Lett. 94, 056804.

Ishii, H., et al., 2003, Nature (London) 426, 540.

Jacobsohn, L., R. Schulze, M. M. da Costa, and M. Nastasi, 2004, Surf. Sci. 572, 418.

Jaffrennou, P., J. Barjon, J.-S. Lauret, B. Attal-Trétout, F. Ducastelle, and A. Loiseau, 2007, J. Appl. Phys. 102, 116102.

Jorio, A., M. Dresselhaus, and G. Dresselhaus, 2008, Carbon Nanotubes: Advanced Topics in the Synthesis, Structure, Properties and Applications (Springer-Verlag, Heidelberg).

Jorio, A., R. Saito, J. H. Hafner, C. M. Lieber, M. Hunter, T. McClure, G. Dresselhaus, and M. S. Dresselhaus, 2001, Phys. Rev. Lett. 86, 1118.

Kalbac, M., H. Farhat, L. Kavan, J. Kong, and M. S. Dresselhaus, 2008, Nano Lett. 8, 3532.

Kalbac, M., L. Kavan, and L. Dunsch, 2009, J. Phys. Chem. C 113, 1340. 
Kang, H. S., and S. Jeong, 2004, Phys. Rev. B 70, 233411.

Kataura, H., Y. Kumazawa, N. Kojima, Y. Maniwa, I. Umezu, S. Masubuchi, S. Kazama, Y. Ohtsuka, S. Suzuki, and Y. Achiba, 2000, Mol. Cryst. Liq. Cryst. 340, 757.

Keast, V., A. Scott, R. Brydson, D. Williams, and J. Bruley, 2001, J. Microsc. 203, 135.

Kelly, K., I. Chiang, E. Mickelson, R. Hauge, J. Margrave, X. Wang, G. Scuseria, C. Radloff, and N. Halas, 1999, Chem. Phys. Lett. 313, 445.

Keskar, G., R. Rao, J. Luo, J. Hudson, J. Chen, and A. Rao, 2005, Chem. Phys. Lett. 412, 269.

Kim, P., T. Odom, J. Huang, and C. Lieber, 2000, Carbon 38, 1741.

Kim, S., J. Lee, C. Na, J. Park, K. Seo, and B. Kim, 2005, Chem. Phys. Lett. 413, 300.

Kitiyanan, B., W. E. Alvarez, J. H. Harwell, and D. E. Resasco, 2000, Chem. Phys. Lett. 317, 497.

Kohn, W., 1983, Theory of the Inhomogeneous Electron Gas (Plenum, New York).

Kohn, W., 1999, Rev. Mod. Phys. 71, 1253.

Komatsu, T., 2001, J. Mater. Chem. 11, 799.

Kramberger, C., H. Rauf, H. Shiozawa, M. Knupfer, B. Büchner, T. Pichler, D. Batchelor, and H. Kataura, 2007, Phys. Rev. B 75, 235437.

Kramberger, C., et al., 2008, Phys. Rev. Lett. 100, 196803.

Kresse, G., and J. Furthmuller, 1996, Phys. Rev. B 54, 11169.

Krivanek, O. L., N. Dellby, and A. R. Lupini, 1999, Ultramicroscopy $\mathbf{7 8}, 1$.

Kroke, E., and M. Schwarz, 2004, Coord. Chem. Rev. 248, 493.

Krstić, V., S. Blumentritt, J. Muster, S. Roth, and A. Rubio, 2003, Phys. Rev. B 67, 041401(R).

Krstić, V., G. L. J. A. Rikken, P. Bernier, S. Roth, and M. Glerup, 2007, Phys. Lett. 77, 107.

Kukovecz, A., T. Pichler, R. Pfeiffer, C. Kramberger, and H. Kuzmany, 2003, Phys. Chem. Chem. Phys. 5, 582.

Kukovecz, A., T. Pichler, R. Pfeiffer, and H. Kuzmany, 2002, Chem. Commun. 1730, 38.

Lambin, P., G. Mark, V. Meunier, and L. Biró, 2003, Int. J. Quantum Chem. 95, 493.

Lammert, P., V. Crespi, and A. Rubio, 2001, Phys. Rev. Lett. 87, 136402.

Latil, S., S. Roche, D. Mayou, and J. C. Charlier, 2004, Phys. Rev. Lett. 92, 256805.

Latil, S., F. Triozon, and S. Roche, 2005, Phys. Rev. Lett. 95, 126802.

Lauret, J. S., R. Arenal, F. Ducastelle, A. Loiseau, M. Cau, B. Attal-Tretout, E. Rosencher, and L. Goux-Capes, 2005, Phys. Rev. Lett. 94, 037405.

Leapman, R. D., P. L. Fejes, and J. Silcox, 1983, Phys. Rev. B 28, 2361.

Lee, C., S. Choi, S. Choi, and S. Hong, 2006, Curr. Appl. Phys. 6, 166.

Lee, R. S., J. Gavillet, M. L. dela Chapelle, A. Loiseau, J.-L. Cochon, D. Pigache, J. Thibault, and F. Willaime, 2001, Phys. Rev. B 64, 121405(R).

Lee, R. S., H. J. Kim, J. E. Fischer, A. Thess, and R. E. Smalley, 1997, Nature (London) 388, 854.

Lherbier, A., X. Blase, Y.-M. Niquet, F. Triozon, and S. Roche, 2008, Phys. Rev. Lett. 101, 036808.

Li, F., Y. Xia, M. Zhao, X. Liu, B. Huang, Y. Ji, and C. Song, 2006, Phys. Lett. A 357, 369.

Li, L.-J., M. Glerup, A. Khlobystov, J. Wiltshire, J.-L. Sauvajol, R. Taylor, and R. J. Nicholas, 2006, Carbon 44, 2752.
Lim, S. H., H. I. Elim, X. Y. Gao, A. T. S. Wee, W. Ji, J. Lee, and J. Lin, 2006, Phys. Rev. B 73, 045402.

Lin, H., R. Arenal, S. Enouz-Vedrenne, O. Stephan, and A. Loiseau, 2009, J. Phys. Chem. C 113, 9509.

Lin, H., J. Lagoute, C. Chacon, R. Arenal, O. Stephan, V. Repain, Y. Girard, S. Enouz, L. Bresson, S. Rousset, and A. Loiseau, 2008, Phys. Status Solidi B 245, 1986.

Liu, A. Y., R. M. Wentzcovitch, and M. L. Cohen, 1989, Phys. Rev. B 39, 1760.

Liu, X., T. Pichler, M. Knupfer, J. Fink, and H. Kataura, 2004, Phys. Rev. B 70, 205405.

Liu, X. M., H. E. Romero, H. R. Gutierrez, K. Adu, and P. C. Eklund, 2008, Nano Lett. 8, 2613.

Liu, Y., and H. Guo, 2004, Phys. Rev. B 69, 115401.

Loiseau, A., F. Willaime, N. Demoncy, G. Hug, and H. Pascard, 1996, Phys. Rev. Lett. 76, 4737.

Ma, J., S. Guan, and C. H. Lai, 2006, Phys. Rev. B 74, 205401. Maciel, I., N. Anderson, M. Pimenta, A. Hartschuh, H. Quian, M. Terrones, H. Terrones, J. Campos-Delgado, A. Rao, L. Novotny, and A. Jorio, 2008, Meteorit. Planet. Sci. 7, 878.

Maguer, A., R. Arenal, P. Jaffrennou, J. L. Cochon, L. Bresson, E. Doris, C. Mioskowski, and A. Loiseau, 2007, J. Nanosci. Nanotechnol. 7, 3528.

Maniwa, Y., K. Mytsuda, H. Kyakuno, S. Ogasawara, T. Hibi, H. Kadowaki, S. Suzuki, Y. Achiba, and H. Kataura, 2007, Nature Mater. 6, 135.

Marini, A., 2008, Phys. Rev. Lett. 101, 106405.

Marinopoulos, A., L. Wirtz, A. Marini, V. Olevano, A. Rubio, and L. Reining, 2004, Appl. Phys. A: Mater. Sci. Process. 78, 1157.

Marinopoulos, A. G., L. Reining, V. Olevano, A. Rubio, T. Pichler, X. Liu, M. Knupfer, and J. Fink, 2002, Phys. Rev. Lett. 89, 076402.

Marques, M., C. Ulrich, F. Nogueira, A. Rubio, K. Burke, and E. Gross, 2006, Time Dependent Density Functional Theory, Lecture Notes in Physics No. 706 (Springer, New York).

Mazzoni, M. S. C., R. W. Nunes, S. Azevedo, and H. Chacham, 2006, Phys. Rev. B 73, 073108.

McGuire, K., N. Gothard, P. Gai, M. Dresselhaus, G. Sumanasekera, and A. Rao, 2005, Carbon 43, 219.

Mele, E. J., and J. J. Ritsko, 1979, Phys. Rev. Lett. 43, 68.

Mele, E. J., and J. J. Ritsko, 1981, Phys. Rev. B 24, 1000.

Meyer, J. C., M. Paillet, G. S. Duesberg, and S. Roth, 2007, Ultramicroscopy 107, 72.

Min, Y.-S., E. J. Bae, I. P. Asanov, U. J. Kim, and W. Park, 2007, Nanotechnology 18, 285601.

Mintmire, J. W., B. I. Dunlap, and C. T. White, 1992, Phys. Rev. Lett. 68, 631.

Miyamoto, Y., A. Rubio, M. L. Cohen, and S. G. Louie, 1994, Phys. Rev. B 50, 4976.

Miyamoto, Y., A. Rubio, S. G. Louie, and M. L. Cohen, 1994, Phys. Rev. B 50, 18360.

Miyata, Y., K. Yanagi, Y. Maniwa, T. Tanaka, and H. Kataura, 2008, J. Phys. Chem. C 112, 15997.

Muller, D. A., D. A. Fitting Kourkoutis, M. Murfitt, J. H. Song, H. Hwang, J. Silcox, N. Dellby, and O. Krivanek, 2008, Science 319, 1073.

Murata, N., J. Haruyama, J. Reppert, A. M. Rao, T. Koretsune, S. Saito, M. Matsudaira, and Y. Yagi, 2008, Phys. Rev. Lett. 101, 027002.

Museur, L., and A. Kanaev, 2008, J. Appl. Phys. 103, 103520. Odom, T., J. Huang, and C. Lieber, 2002, J. Phys.: Condens. Matter 14, R145. 
Onida, G., L. Reining, and A. Rubio, 2002, Rev. Mod. Phys. 74, 601.

Orlikowski, D., M. Nardelli, J. Bernholc, and C. Roland, 2000, Phys. Rev. B 61, 14194.

Owens, F. J., 2007, Nanoscale Res. Lett. 2, 447.

Panchakarla, L. S., A. Govindaraj, and C. N. R. Rao, 2007, ACS Nano 1, 494.

Park, C.-H., C. D. Spataru, and S. G. Louie, 2006, Phys. Rev. Lett. 96, 126105.

Pichler, T., A. Kukovecz, H. Kuzmany, H. Kataura, and Y. Achiba, 2003, Phys. Rev. B 67, 125416.

Qin, L.-C., 2006, Rep. Prog. Phys. 69, 2761.

Qin, L.-C., 2007, Phys. Chem. Chem. Phys. 9, 31.

Quandt, A., C. Özdogbreve, J. Kunstmann, and H. Fehske, 2008, Physica Status Solidi B 245, 2077.

Radosavljević, M., J. Appenzeller, V. Derycke, R. Martel, P. Avouris, A. Loiseau, J. Cochon, and D. Pigache, 2003, Appl. Phys. Lett. 82, 4131.

Rao, A., P. Eklund, S. Bandow, A. Thess, and R. Smalley, 1997, Nature (London) 388, 257.

Rauf, H., T. Pichler, M. Knupfer, J. Fink, and H. Kataura, 2004, Phys. Rev. Lett. 93, 096805.

Reyes-Reyes, M., N. Grobert, R. Kamalakaran, T. Seeger, D. Golberg, M. Rühle, Y. Bando, M. Terrones, and M. Terrones, 2004, Chem. Phys. Lett. 396, 167.

Robertson, J., 2002, Mater. Sci. Eng. R. 37, 129.

Rocha, A. R., J. E. Padilha, A. Fazzio, and A. J. R. da Silva, 2008, Phys. Rev. B 77, 153406.

Rossato, J., R. J. Baierle, T. M. Schmidt, and A. Fazzio, 2008, Phys. Rev. B 77, 035129.

Rubio, A., 1997a, in Nanowires, edited by P. A. Serena and N. García, Proceedings of the NATO Advanced Science Institute (Kluwer Academic, Dordrecht, The Netherlands), pp. 133-142.

Rubio, A., 1997b, Condens. Matter News 6, 6.

Rubio, A., 1999, Appl. Phys. A: Mater. Sci. Process. 68, 275.

Rubio, A., J. L. Corkill, and M. L. Cohen, 1994, Phys. Rev. B 49, 5081.

Rümmeli, M. H., et al., 2006, Nanotechnology 17, 5469.

Saito, R., G. Dresselhaus, and M. Dresselhaus, 1998, Physical

Properties of Carbon Nanotubes (Imperial College Press, London).

Saito, S., 1996, Science 76, 971.

Saito, Y., T. Yoshikawa, S. Bandow, M. Tomita, and T. Hayashi, 1993, Phys. Rev. B 48, 1907.

Scherzer, O., 1949, J. Appl. Phys. 20, 20.

Serin, V., R. Brydson, A. Scott, Y. Kihn, O. Abidate, B. Maquin, and A. Derre, 2000, Carbon 38, 547.

Shimoyama, I., G. Wu, T. Sekiguchi, and Y. Baba, 2000, Phys. Rev. B 62, R6053.

Shirasaki, T., A. Derré, M. Ménétrier, A. Tressaud, and S. Flandrois, 2000, Carbon 38, 1461.

Silly, M. G., P. Jaffrennou, J. Barjon, J.-S. Lauret, F. Ducastelle, A. Loiseau, E. Obraztsova, B. Attal-Tretout, and E. Rosencher, 2007, Phys. Rev. B 75, 085205.

Smith, D., 2008, Ultramicroscopy 108, 159.

Spataru, C. D., S. Ismail-Beigi, L. X. Benedict, and S. G. Louie, 2004, Phys. Rev. Lett. 92, 077402.

Spătaru, N., X. Zhang, T. Spataru, D. A. Tryk, and A. Fujishima, 2008, J. Electrochem. Soc. 155, B264.

Srivastava, D., M. Menon, C. Daraio, S. Jin, B. Sadanadan, and A. M. Rao, 2004, Phys. Rev. B 69, 153414.

Stephan, O., and P. Ajayan, 1994, Science 266, 1683.
Strinati, G., 1984, Phys. Rev. B 29, 5718.

Sumpter, B. G., J. Huang, V. Meunier, J. M. Romo-Herrera, E. Cruz-Silva, H. Terrones, and M. Terrones, 2009, Int. J. Quantum Chem. 109, 97.

Suzuki, S., F. Maeda, Y. Watanabe, and T. Ogino, 2003, Phys. Rev. B 67, 115418.

Tanaka, T., H. Jin, Y. Miyata, S. Fujii, H. Suga, Y. Naitoh, T. Minari, T. Miyadera, K. Tsukagoshi, and H. Kataura, 2009, Nano Lett. 9, 1497.

Tans, S. J., and C. Dekker, 2000, Nature (London) 404, 834.

Tarrio, C., and S. E. Schnatterly, 1989, Phys. Rev. B 40, 7852.

Taylor, C. A., II, S. W. Brown, V. Subramaniam, S. Kidner, S.

Rand, and R. Clarke, 1994, Appl. Phys. Lett. 65, 1251.

Tenne, R., and A. Zettl, 2001, Carbon Nanotubes 80, 81.

Terrones, M., N. Grobert, and H. Terrones, 2002, Carbon 40, 1665.

Terrones, M., A. Jorio, M. Endo, A. Rao, Y. Kim, T. Hayashi, H. Terrones, J.-C. Charlier, G. Dresselhaus, and M. Dresselhaus, 2004, Mater. Today 7, 30.

Terrones, M., R. Kamalakaran, R. Seeger, and M. Rühle, 2000, Chem. Commun. (Cambridge) 23, 2325.

Terrones, M., H. Terrones, F. Banhart, J. Charlier, and P. M. Ajayan, 2000, Science 288, 1226.

Terrones, M., H. Terrones, N. Grobert, W. K. Hsu, Y. Q. Zhu, J. P. Hare, H. W. Kroto, D. R. M. Walton, P. Kohler-Redlich, M. Rühle, J. P. Zhang, and A. K. Cheetham, 1999, Appl. Phys. Lett. 75, 3932.

Teter, D. M., and R. J. Hemley, 1996, Science 271, 53.

Thomsen, C., and S. Reich, 2000, Phys. Rev. Lett. 85, 5214.

Tsang, J., M. Freitag, V. Perebeinos, J. Liu, and P. Avouris, 2007, Nat. Nanotechnol. 2, 725.

Villalpando-Paez, F., et al., 2006, Chem. Phys. Lett. 424, 345.

Wang, F., L. Dukovic, L. Brus, and T. Heinz, 2005, Science 308, 838.

Wang, W., X. Bai, K. Liu, Z. Xu, D. Golberg, Y. Bando, and E. Wang, 2006, J. Am. Chem. Soc. 128, 6530.

Watanabe, M. O., S. Itoh, T. Sasaki, and K. Mizushima, 1996, Phys. Rev. Lett. 77, 187.

Wiltshire, J. G., L. J. Li, L. M. Herz, R. J. Nicholas, M. Glerup, J. L. Sauvajol, and A. N. Khlobystov, 2005, Phys. Rev. B 72, 205431.

Wirtz, L., A. Marini, M. Gruning, and A. Rubio, 2005, e-print arXiv:cond-mat/0508421.

Wirtz, L., A. Marini, and A. Rubio, 2006, Phys. Rev. Lett. 96, 126104.

Wirtz, L., and A. Rubio, 2003, AIP Conf. Proc. 685, 402.

Wirtz, L., and A. Rubio 2010, unpublished.

Wirtz, L., A. Rubio, R. A. de la Concha, and A. Loiseau, 2003, Phys. Rev. B 68, 045425.

Wu, J., W.-Q. Han, W. Walukiewicz, J. W. Ager, W. Shan, E. E. Haller, and A. Zettl, 2004, Nano Lett. 4, 647.

Xiao, K., Y. Liu, P. Hu, G. Yu, W. Hu, D. Zhu, X. Liu, H. Liu, and D. Wu, 2006, Appl. Phys. A: Mater. Sci. Process. 83, 53. Xu, F., M. Minniti, P. Barone, A. Sindona, A. Bonano, and A. Oliva, 2008, Carbon 46, 1489.

Xu, F., M. Minniti, C. Giallombardo, A. Cupolillo, P. Barone, A. Oliva, and L. Papagno, 2007, Surf. Sci. 601, 2819.

Xu, Y.-N., and W. Y. Ching, 1991, Phys. Rev. B 44, 7787.

Xu, Z., W. Lu, W. Wang, C. Gu, K. Liu, X. Bai, E. Wang, and H. Dai, 2008, Adv. Mater. 20, 3615.

Yanagi, K., T. Iitsuka, S. Fujii, and H. Kataura, 2008, J. Phys. Chem. C 112, 18889.

Yang, C.-K., 2008, Appl. Phys. Lett. 92, 033103. 
Yap, Y. K., 2008, Ed., B-C-N Nanotubes and Related Nanostructures (Springer, New York).

Yi, J.-Y., and J. Bernholc, 1993, Phys. Rev. B 47, 1708.

Yi, S., H. Zhang, G. Zhang, S. Hu, L. Pei, and J. Yin, 2006, Physica B 373, 131.

Yu, D. P., X. S. Sun, C. S. Lee, I. Bello, S. T. Lee, H. D. Gu, K. M. Leung, G. W. Zhou, Z. F. Dong, and Z. Zhang, 1998, Appl. Phys. Lett. 72, 1966.

Yu, P., and M. Cardona, 2001, Fundamentals of Semiconductors, 3rd ed. (Springer, New York).

Yu, S. S., W. T. Zheng, Q. B. Wen, and Q. Jiang, 2008, Carbon 46, 537.
Zanchetta, J., and A. Marchand, 1965, Carbon 3, 332.

Zhang, J., K. P. Loh, J. Zheng, M. B. Sullivan, and P. Wu, 2007, Phys. Rev. B 75, 245301.

Zhang, W. J., J. Y. Zhang, P. J. Li, X. Shen, Q. F. Zhang, and J. L. Wu, 2008, Nanotechnology 19, 085202.

Zhou, Z., X. Gao, J. Yan, D. Song, and M. Morinaga, 2004a, J. Phys. Chem. B 108, 9023.

Zhou, Z., X. Gao, J. Yan, D. Song, and M. Morinaga, 2004b, Carbon 42, 2677.

Zunger, A., A. Katzir, and A. Halperin, 1976, Phys. Rev. B 13, 5560 . 